DOI 10.4171/JEMS/274

Ehud Hrushovski · Anand Pillay

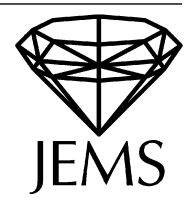

\title{
On NIP and invariant measures
}

Received March 18, 2008 and in revised form January 29, 2009 and November 13, 2010

\begin{abstract}
We study forking, Lascar strong types, Keisler measures and definable groups, under an assumption of NIP (not the independence property), continuing aspects of the paper [16]. Among key results are (i) if $p=\operatorname{tp}(b / A)$ does not fork over $A$ then the Lascar strong type of $b$ over $A$ coincides with the compact strong type of $b$ over $A$ and any global nonforking extension of $p$ is Borel definable over bdd $(A)$, (ii) analogous statements for Keisler measures and definable groups, including the fact that $G^{000}=G^{00}$ for $G$ definably amenable, (iii) definitions, characterizations and properties of "generically stable" types and groups, (iv) uniqueness of invariant (under the group action) Keisler measures on groups with finitely satisfiable generics, (v) a proof of the compact domination conjecture for (definably compact) commutative groups in $o$-minimal expansions of real closed fields.
\end{abstract}

\section{Introduction and preliminaries}

The general theme of this paper is to find and study stable-like behaviour in theories and definable groups without the independence property. This was a theme in the modeltheoretic analysis of algebraically closed valued fields [10], [11], [14]. It was also an aspect of the paper [16], although there the main motivation was to resolve some conjectures about definably compact groups in $o$-minimal structures. In fact a bonus in the current paper is a proof of the compact domination conjecture (formulated in [16]), at least for commutative groups, and various elaborations, which is fairly direct but also uses some of the general machinery we develop.

Both authors have been a bit slow to realize the relevance of Shelah's notion of forking to theories with NIP. We rectify this in the current paper where we will be quite explicit about the role of forking.

Note that a theory $T$ is stable if and only if it is simple and has NIP. In stable theories we have on the one hand the "algebraic" behaviour of nonforking independence, namely symmetry, transitivity, existence of nonforking extensions, as well as local character (any type does not fork over some small set). On the other hand we have (again in stable theories) what can be called broadly "multiplicity theory", the structure and behaviour of

E. Hrushovski: Hebrew University (Giv'at Ram), Jerusalem 91904, Israel; e-mail: ehud@math.huji.ac.il

A. Pillay: Department of Pure Mathematics, University of Leeds, Leeds LS2 9JT, UK; e-mail: pillay@maths.leeds.ac.uk 
nonforking extensions of a type. Included in "multiplicity theory" are alternative characterizations of nonforking, for example a global type $p$ does not fork over a model $M$ iff $p$ is definable over $M$ iff $p$ is finitely satisfiable in $M$ iff $p$ is $\operatorname{Aut}(\bar{M} / M)$-invariant (does not split over $M$ ). Included also is the finite equivalence relation theorem: a type over an algebraically closed set is stationary, namely has a unique global nonforking extension. In the early texts on stability theory (the original papers and book of Shelah, but also the treatment by Lascar and Poizat), the proofs and development of the algebraic properties of forking were tied up with multiplicity theory (in the form of heirs, coheirs for example). In the work on simple theories, the two strands were distinguished, the algebraic theory being valid in all simple theories, but not the latter. Multiplicity theory did make an appearance in simple theories, but in the (weak) form of the "independence theorem over a model" and more generally over boundedly closed (rather than algebraically closed) sets. As the validity of the algebraic theory of forking is characteristic of simple theories, it will fail for unstable theories with NIP. One of the points of the current paper is to recover aspects of the multiplicity part of stability theory for theories with NIP. Invariance (rather than stationarity) turns out to be important and already invariant types played a role in the analysis of algebraically closed valued fields. We show for example (in Section 2), extending work of Shelah [35] and Adler [1], that assuming NIP if $p$ is a global type and $A$ a small set then $p$ does not fork over $A$ iff $p$ is $\operatorname{Aut}(\bar{M} / \operatorname{bdd}(A))$ invariant iff $p$ is "Borel definable" over $\operatorname{bdd}(A)$. Keisler measures figured a lot in the earlier paper [16] and we entertained the possibility that replacing types by Keisler measures would give a smoother theory and better chance of recovering stationary-like behaviour (uniqueness of nonforking extensions). In Section 4 the results for types are carried over to Keisler measures. This includes the "Borel definability" over $\operatorname{bdd}(A)$ of a measure which does not fork over $A$, the proof of which uses the Vapnik-Chervonenkis theorem. We reduce measures to types in two ways. (a) If the Keisler measure is $A$ invariant then it corresponds to a Borel measure on the space of bdd $(A)$-invariant types (see 4.6). (b) An invariant Keisler measure is also the "frequency average" of some sequence of invariant types (see 4.8). Though these representations of Keisler measures might seem to make considerations of measures unnecessary, in fact some of our proofs of facts about types go through measures. This point appears in Section 5, where among other things we show that definable amenability (the existence of left invariant measures) of a definable group $G$ is equivalent to the existence of generic types in the sense of forking.

In Section 3 we study what we call generically stable types (and what Shelah has just called stable types), mainly in an NIP context. These are types whose behaviour vis-à-vis nonforking extensions is like that in stable theories, namely a global nonforking extension is both definable and finitely satisfiable. A special case of a generically stable type is a stably dominated type, as introduced in [11], and our results place those of [11] in the appropriate general context. Likewise generically stable groups, studied in Section 6, generalize the stably dominated groups of [14].

In Section 5 we recall the groups with $f s g$ (finitely satisfiable generics) which were introduced in [16], and generalize some results from [16] such as definable amenability, to definable groups with generic types in the sense of forking. 
Definably compact groups in a variety of settings ( $o$-minimal, $P$-minimal, metastable) have either been proved to have or are expected to have $f s g$. In Section 7 we prove the uniqueness of translation invariant Keisler measures in $f s g$ groups. We see this as a kind of common generalization of the uniqueness of global translation invariant types for stable groups, and of the uniqueness of Haar measure for compact groups; this exemplifies our search for stable-like behaviour at the level of Keisler measures.

It is natural to try to both extend the notion of generic stability from types to measures, as well as to find group-free versions of the $f_{s} g$ property. This is discussed briefly in Sections 4 and 7, and the problems will be addressed in a future paper with P. Simon.

Section 8 is devoted to a proof of compact domination for commutative definably compact groups in $o$-minimal expansions of real closed fields. In fact we prove a strong version, domination of $G$ by a "semi- $o$-minimal" compact Lie group $G / G^{00}$. Direct $o$ minimal style arguments as well as some of the general theory of invariant types play a role in the proof. Our proof makes use of a theorem on the existence of definable Skolem functions for " $o$-minimal subsets of finite-dimensional groups", which is proved in the appendix.

Much inspiration for our work on measures comes from Keisler's work [20] and [21]. But our emphasis differs from Keisler's. Among the main points of Keisler is that if $\phi(x, y)$ is a stable formula, then any $\phi$-measure is a "convergent weighted sum" of $\phi$ types. (Here a $\phi$-measure over $M$ is a finitely additive measure on the Boolean algebra generated by formulas of the form $\phi(x, a)$ for $a \in M$.) So all the properties of nonforking in a stable theory (symmetry, stationarity of types over models,...) pass automatically to measures. Keisler does consider measures in (possibly unstable) theories with NIP. The good class of measures he identifies are so-called "smooth" or "minimally unstable" measures. Loosely (and possibly incorrectly) speaking, a smooth measure over $M$ is a measure over $M$ which has a unique extension to the "unstable part" of $\bar{M}$. He proves that such measures exist. So the only freedom in taking extensions of a smooth measure is with respect to stable formulas, and thus in a sense forking theory for smooth measures essentially reduces to forking theory for measures in stable theories. On the other hand our main focus will be global measures which are invariant over some small set. Only in special cases will these also be smooth.

We will use standard notation. Namely $T$ denotes a complete theory in a language $L$ and we work in a saturated model $\bar{M}$ of $T$. The letters $x, y, z, \ldots$ usually denote finite tuples of variables, $A, B, C$ small subsets of $\bar{M}$ and $M, N, \ldots$ small elementary submodels of $\bar{M}$. There is no harm in working in $\bar{M}^{\text {eq }}$, but sometimes we will assume our theory is one-sorted. A reader would benefit from having some familiarity with stability theory and stable group theory, a reference for which is the first chapter of [32]. However the paper is reasonably self-contained and can on the whole be read independently of [16].

When it is convenient we denote the space of complete types over $A$ in free variable $x$ by $S_{x}(A)$. By a global (complete) type we mean some $p(x) \in S(\bar{M})$. Recall that a partial type $\Sigma(x)$ (over some set of parameters and closed under finite conjunctions) is said to divide over a set $A$ if there is $\phi(x, b) \in \Sigma(x)$ and an $A$-indiscernible sequence $\left(b_{i}: i<\omega\right)$ of realizations of $\operatorname{tp}(b / A)$ such that $\left\{\phi\left(x, b_{i}\right): i<\omega\right\}$ is inconsistent. $\Sigma(x)$ is said to fork over $A$ if $\Sigma$ implies some finite disjunction of formulas, each of which 
divides over $A$. Note that if $p(x)$ is a complete type over some $|A|^{+}$-saturated model $M$ and $A \subseteq M$ then $p(x)$ divides over $A$ iff $p(x)$ forks over $A$. Also if $A$ is any (small) set of parameters, and $p(x) \in S(A)$, then $p$ does not fork over $A$ if and only if $p(x)$ has a global extension which does not divide (equivalently does not fork) over $A$.

A rather more basic notion is splitting: Let $p(x) \in S(B)$ and $A \subseteq B$. We say that $p$ does not split over $A$ if for any $L$-formula $\phi(x, y)$ and $b, c \in B$, if $\operatorname{tp}(b / A)=\operatorname{tp}(c / A)$ then $\phi(x, b) \in p$ iff $\phi(x, c) \in p$. This tends to be more meaningful when $B$ is some $|A|^{+}$-saturated model. Even more basic notions are finite satisfiability and heir: Again for $p(x) \in S(B)$ and $A \subseteq B, p$ is said to be finitely satisfiable in $A$ if any formula in $p$ is satisfied by some tuple from $A$. And $p$ is said to be an heir of $p \mid A$ if for any $L_{A}$-formula $\phi(x, y)$ (that is, $\phi$ has parameters from $A$ ) and $b$ from $B$, if $\phi(x, b) \in p$ then $\phi\left(x, b^{\prime}\right) \in p$ for some $b^{\prime}$ from $A$. Here usually $B$ is a model.

We will be discussing various kinds of strong types, so let us fix notation. First by $\operatorname{Aut}(\bar{M} / A)$ we mean the group of automorphisms of $\bar{M}$ which fix $A$ pointwise. Two tuples (of the same length or elements of the same sort) $a$ and $b$ are said to have the same strong type over a set $A$ of parameters ( $\operatorname{symbolically} \operatorname{stp}(a / A)=\operatorname{stp}(b / A))$ if $E(a, b)$ for each finite $A$-definable equivalence relation $E$ on the relevant sort (where finite means having only finitely many classes). It is well-known that $\operatorname{stp}(a / A)=\operatorname{stp}(b / A)$ if and only if $a$ and $b$ have the same type over acl $(A)$ where acl $(-)$ is computed in $\bar{M}^{\text {eq }}$, and so in fact we can just define the strong type of $a$ over $A$, $\operatorname{stp}(a / A)$, to be $\operatorname{tp}(a / \operatorname{acl}(A))$. To be consistent with later notation it might be better to call strong types Shelah strong types or profinite strong types.

We say that $a$ and $b$ have the same compact strong type or KP strong type over $A$ if $E(a, b)$ for each bounded equivalence relation $E$ on the relevant sort which is typedefinable over $A$, that is, defined by a possibly infinite set of formulas over $A$. Here bounded means having strictly less than $|\bar{M}|$-many classes, which is equivalent to having at most $2^{|T|+|A|}$-many classes. An (Ø-) hyperimaginary $e$ is by definition the class of a $\emptyset$-type-definable equivalence relation. We define $\operatorname{bdd}(A)$ to be the set of hyperimaginaries which have small orbit under $\operatorname{Aut}(\bar{M} / A)$. It follows from [22] (see 4.18 there) that $a$ and $b$ have the same $K P$ strong type over $A$ iff they have the same type over $\operatorname{bdd}(A)$ (where types over hyperimaginaries are made sense of in [12] for example). In particular if $a$ and $b$ have the same $K P$ strong type over $A$, then for any $A$-type-definable set $X$ to which $a, b$ belong and bounded $A$-type-definable equivalence relation $E$ on $X$, $a$ and $b$ are in the same $E$-class. All this makes sense for type-definable equivalence relations on sets of infinite tuples, although in [22] we pointed out that a bounded infinitary hyperimaginary is "equivalent" to a sequence of bounded finitary hyperimaginaries. In addition to the papers cited above, a good reference for hyperimaginaries is Wagner's book [40].

Finally we say that tuples $a$ and $b$ have the same Lascar strong type over $A$ if $E(a, b)$ for any bounded equivalence relation $E$ which is invariant under $\operatorname{Aut}(\bar{M} / A)$. The relation "equality of Lascar strong type over $A$ " is the transitive closure of the relation $R_{A}(-,-)$, where $R_{A}(x, y)$ says: $x$ and $y$ are elements of some infinite $A$-indiscernible sequence. Following Lascar we let $\operatorname{Autf}(\bar{M} / A)$ denote the group of automorphisms of $\bar{M}$ which fix all Lascar strong types over $A$. Tuples $a, b$ from $\bar{M}$ (which are possibly infinite) will 
have the same Lascar strong type over $A$ if and only if they are in the same orbit under $\operatorname{Autf}(\bar{M} / A)$.

Note that Lascar strong type refines $K P$ strong type refines strong type refines type (all over $A$ ). If $A=M$ is a model, Lascar strong type coincides with type. In a stable theory Lascar strong types over $A$ coincide with strong types over $A$. This is conjectured to be true in simple theories too, and was proved in the supersimple case [5]. In the general simple case we only know that Lascar strong types coincide with $K P$ strong types. In any case in the current paper we will consider such questions for theories with NIP.

We will also be referring to various kinds of "connected components" of a definable (or even type-definable group) $G$. Suppose $A$ includes the parameters over which $G$ is (type)-defined. Let $G_{A}^{0}$ be the intersection of all (relatively) $A$-definable subgroups of $G$ of finite index. Let $G_{A}^{00}$ denote the smallest type-definable (over $A$ ) subgroup of $G$ of "bounded" index (equivalently of index at most $2^{|L|+|A|}$ ). Let $G_{A}^{000}$ denote the smallest subgroup of $G$ of bounded index which is $\operatorname{Aut}(\bar{M} / A)$-invariant. If for example $G_{A}^{0}$ does not depend on $A$, but only on $G$, we say that $G^{0}$ exists. Likewise for $G^{00}$ and $G^{000}$.

There is an analogy between definable groups $G$ (and their quotients such as $G / G^{00}$ etc.) and automorphism groups (and their corresponding quotients) which permeates this paper. We take the liberty to state for the record a couple of constructions which produce automorphism groups from definable group actions. The first is fairly well-known, and the second, appearing in the first author's Ph.D. thesis in the stable case, is less so.

Construction C1. Let $M$ be a structure (in a language $L$ ) containing among other things a $\emptyset$-definable group $G$. Let $M^{\prime}$ be the structure obtained by adjoining a new sort $S$ to $M$, together with a regular (strictly transitive) action of $G$ on $S$.

Let $\operatorname{Aut}_{M^{\prime}}(S)$ be the group of permutations of $S$ induced by automorphisms of $M^{\prime}$, and $\operatorname{Aut}_{M^{\prime}}(S / G)$ its normal subgroup consisting of permutations of $S$ induced by automorphisms of $M^{\prime}$ which fix $G$ pointwise. Then $\operatorname{Aut}_{M^{\prime}}(S / G)$ is (essentially by definition) the group of permutations of $S$ which commute with the action of $G$, which we call $G^{*}$. As the action is regular, $G^{*}$ is (noncanonically) anti-isomorphic to $G$ (so also isomorphic to $G)$. Namely, for any $a \in S$ define an anti-isomorphism $h_{a}: G^{*} \rightarrow G$ by: for $\sigma \in G^{*}$, $h_{a}(\sigma)$ is the unique $g \in G$ such that $\sigma(a)=g \cdot a$. Moreover $\operatorname{Aut}_{M^{\prime}}(S)$ is the semidirect product of $\operatorname{Aut}_{M}(G)$ and $G^{*}$.

Construction C2. Let $M$ be a structure in a language $L$ which now includes a $\emptyset$-definable transitive group action of a group $G$ on a set $X$ (written as $(g, x) \mapsto g \cdot x$ ). Assume moreover that for some finite subset $\left\{a_{1}, \ldots, a_{m}\right\}$ of $X$ the pointwise stabilizer in $G$ of this finite subset is the identity. Let $M^{\prime}$ (in a language $L^{\prime}$ ) be obtained by adjoining a new sort $S$ and a bijection $h$ between $X$ and $S$.

Let $M^{\prime \prime}$ be the "reduct" of $M^{\prime}$ containing $M$, with its $L$-structure, the new sort $S$, and the single relation $R \subseteq X^{m+1} \times S^{m+1}$ where

$$
\begin{array}{r}
R=\left\{\left(b_{1}, \ldots, b_{m}, b_{m+1}, h\left(g \cdot b_{1}\right), \ldots, h\left(g \cdot b_{m}\right), h\left(g \cdot b_{m+1}\right)\right):\right. \\
\left.b_{1}, \ldots, b_{m+1} \in X, g \in G\right\} .
\end{array}
$$

This $M^{\prime \prime}$ is the structure we want to consider. 
Note that $R$ is $\emptyset$-definable in $M^{\prime}$. On the other hand note that the map $h$ is definable in $M^{\prime \prime}$ over the parameters $\left\{a_{1}, \ldots, a_{m}, h\left(a_{1}\right), \ldots, h\left(a_{m}\right)\right\}$ in $M^{\prime \prime}$ : for any $a \in X, h(a)$ is the unique $b \in S$ such that $R\left(a_{1}, \ldots, a_{m}, a, h\left(a_{1}\right), \ldots, h\left(a_{m}\right), b\right)$.

Let us also write (hopefully without ambiguity) $(g, s) \mapsto g \cdot s$ for the action of $G$ on $S$ induced by the action of $G$ on $X$ and the bijection $h$. (Namely $(g \cdot s)=h\left(g \cdot h^{-1}(s)\right)$.)

Claim C2(i). $\operatorname{Aut}_{M^{\prime \prime}}(S / X)=G$.

Explanation. For any $g \in G$, let $\sigma_{g}$ be the permutation of $M^{\prime \prime}$ which is the identity on $M$ and acts as $g$ on $S$. Then clearly $\sigma_{g}$ preserves the relation $R$, hence is an automorphism of $M^{\prime \prime}$. Conversely, suppose $\sigma \in \operatorname{Aut}_{M^{\prime \prime}}(S / X)$. To make notation less cumbersome, write $a^{\prime}$ for $h(a)$ (when $a \in X$ ). Let $b \in X$ be arbitrary, and consider the tuple $\left(a_{1}, \ldots, a_{m}, b, a_{1}^{\prime}, \ldots, a_{m}^{\prime}, b^{\prime}\right)$. It belongs to the relation $R$. Hence $\left(a_{1}, \ldots, a_{m}, b\right.$, $\left.\sigma\left(a_{1}^{\prime}\right), \ldots, \sigma\left(a_{m}^{\prime}\right), \sigma\left(b^{\prime}\right)\right) \in R$. So by definition of $R$, there is $g \in G$ such that $\sigma\left(a_{i}^{\prime}\right)=$ $\left(g \cdot a_{i}\right)^{\prime}=g \cdot a_{i}^{\prime}$ for each $i=1, \ldots, m$ and also $\sigma\left(b^{\prime}\right)=(g \cdot b)^{\prime}=g \cdot b^{\prime}$. We know that $g$ is in fact the unique element of $G$ taking $a_{1}^{\prime}, \ldots, a_{m}^{\prime}$ to $\sigma\left(a_{1}^{\prime}\right), \ldots, \sigma\left(a_{m}^{\prime}\right)$. As $b \in X$ was arbitrary this shows that $\sigma$ acts on $S$ as the element $g \in G$ does.

Finally we leave it to the reader to see that:

Claim C2(ii). $\operatorname{Aut}_{M^{\prime \prime}}(S)$ is the semidirect product of $\operatorname{Aut}_{M}(X)$ and $G$.

(End of Constructions $\mathrm{C} 1$ and $\mathrm{C} 2$.)

Fact 1.1. Suppose $T$ has NIP. Then for any formula $\phi(x, y)$, there is $N<\omega$ such that if $\left(a_{i}: i<\omega\right)$ is an indiscernible sequence, then there does not exist $b$ such that $\neg\left(\phi\left(a_{i}, b\right) \leftrightarrow \phi\left(a_{i+1}, b\right)\right)$ for $i=0, \ldots, N-1$. It follows that if $\left(a_{i}: i<\omega\right)$ is totally indiscernible (or an indiscernible set), then for any $b$, either $\left|\left\{i<\omega: \models \phi\left(a_{i}, b\right)\right\}\right| \leq N$ or $\left|\left\{i<\omega: \models \neg \phi\left(a_{i}, b\right)\right\}\right| \leq N$.

At some point we will, assuming $N I P$, refer to $\operatorname{Av}(I / M)$ where $I$ is some infinite indiscernible sequence (with no last element). It is the complete type over $M$ consisting of formulas with parameters from $M$ which are true on a cofinal subset of $I$. This makes sense by Fact 1.1 .

\section{Forking and Lascar strong types}

Forking in NIP theories typically has a different character from forking in simple theories (although the definition, as in the introduction, is the same). In simple theories, forking is associated to a "lowering of dimension". In NIP theories forking can come from just a lowering of order of magnitude within a given dimension. Although dimension is no less important in $C$-minimal and $o$-minimal theories than in strongly minimal ones, we do not at the moment know the right NIP based notion that specializes to lowering of dimension in these cases. (Thorn forking is of course a very useful notion but does not apply to the C-minimal case.)

This section builds on work of Poizat [34], Shelah [36] and Adler [1]. Many of our key notions make an explicit or implicit appearance in Chapter 12 of the Poizat reference. 
For completeness we will begin by restating some of the results by the above mentioned people. The first is a striking characterization of forking in NIP theories from [1] but with roots in [36].

Proposition 2.1. (Assume NIP.) Let $p(x) \in S(\bar{M})$ be a global type and A a (small) set. Then:

(i) $p$ does not fork over $A$ iff $p$ is $\operatorname{Autf}(\bar{M} / A)$-invariant, in other words if $p(x)$ is fixed by any automorphism of $\bar{M}$ which fixes all Lascar strong types over $A$.

(ii) In particular if $A=M$ is a model, then $p$ does not fork over $M$ iff $p$ is invariant under $\operatorname{Aut}(\bar{M} / M)$, in other words $p$ does not split over $M$.

Proof. (i) Right implies left: suppose $\phi(x, y) \in L$ and $\left(b_{i}: i<\omega\right)$ is an $A$-indiscernible sequence of realizations of $\operatorname{tp}(b / A)$ where $\phi(x, b) \in p$. We may assume that $b=b_{0}$. As $\operatorname{Lstp}\left(b_{i} / A\right)=\operatorname{Lstp}\left(b_{0} / A\right)$ for all $i, \phi\left(x, b_{i}\right) \in p(x)$ for all $i$, so trivially $\left\{\phi\left(x, b_{i}\right)\right.$ : $i<\omega\}$ is consistent.

Left implies right: Suppose first that $b_{0}, b_{1}$ are the first two members of an $A$-indiscernible sequence $\left(b_{i}: i<\omega\right)$, and $\phi(x, y) \in L$. We claim that $\phi\left(x, b_{0}\right) \in p$ iff $\phi\left(x, b_{1}\right) \in p$. If not then without loss of generality $\phi\left(x, b_{0}\right) \wedge \neg \phi\left(x, b_{1}\right) \in p$. But note that $\left(\left(b_{i}, b_{i+1}\right): i=0,2,4, \ldots\right)$ is also an $A$-indiscernible sequence. So as $p$ does not divide over $A,\left\{\phi\left(x, b_{i}\right) \wedge \neg \phi\left(x, b_{i+1}\right): i=0,2,4, \ldots\right\}$ is consistent, but this contradicts NIP (see Fact 1.1). So our claim is proved. Now if $\operatorname{Lstp}(b / A)=\operatorname{Lstp}(c / A)$ then we can find $b=b_{0}, b_{1}, \ldots, b_{n}=c$ such that $\left(b_{i}, b_{i+1}\right)$ are the first two members of an $A$-indiscernible sequence, for each $i=0, \ldots, n-1$. So by our claim, $\phi(x, b) \in p$ iff $\phi(x, c) \in p$. This completes the proof of (i).

(ii) is immediate because types over models and Lascar strong types over models coincide.

Definition 2.2. Let $p(x) \in S(\bar{M})$ be a global type.

(i) We say that $p$ is invariant over the small subset $A$ of $\bar{M}$ if $p$ is $\operatorname{Aut}(\bar{M} / A)$-invariant.

(ii) We say that $p$ is invariant if it is invariant over some small set.

Invariant types were studied by Poizat as "special" types. By Proposition 2.1, if $T$ has NIP then the invariant global types coincide with the global types which do not fork over some small set. If the global type $p$ is $A$-invariant then we have a kind of defining schema for $p$, namely for each $\phi(x, y) \in L$ we have some family $D_{p} \phi$ of complete $y$ types over $A$ such that for any $b \in \bar{M}, \phi(x, b) \in p$ iff $\operatorname{tp}(b / A) \in D_{p} \phi$. So we can apply the schema $D_{p}$ to not only supersets $B$ of $A$ living in $\bar{M}$ but also to sets $B \supseteq A$ living in a proper elementary extension $\bar{M}^{\prime}$ of $\bar{M}$. In any case for any such set $B$, by $p \mid B$ we mean the complete type over $B$ resulting from applying the schema $D_{p}$ to $B$. We will see subsequently that under the NIP hypothesis the defining schema $D_{p}$ will be "Borel". Given invariant global types $p(x) \in S(\bar{M}), q(y) \in S(\bar{M})$ we can form the product $p(x) \otimes q(y) \in S_{x y}(\bar{M})$ as follows: Let $\phi(x, y)$ be over $\bar{M}$. We may assume $\phi(x, y)$ to be over small $A$ where both $p, q$ are $A$-invariant. We put $\phi(x, y) \in p(x) \otimes q(y)$ if for some (any) $b$ realizing $q(y) \mid A, \phi(x, b) \in p(x)$. 
Alternatively, if we are willing to consider elements of some $|\bar{M}|^{+}$-saturated model containing $\bar{M}$, define $p(x) \otimes q(y)$ to be $\operatorname{tp}(a, b / \bar{M})$ where $b$ realizes $q(y)$ and $a$ realizes $p \mid(\bar{M} b)$.

Note that if the global types $p(x), q(y)$ are invariant, then so is $p(x) \otimes q(y)$. We see easily that $\otimes$ is associative. However it need not be commutative. Namely considering both $p(x) \otimes q(y)$ and $q(y) \otimes p(x)$ as elements of $S_{x y}(\bar{M})$, they may not be equal.

For an invariant global type $p(x)$, and disjoint copies $x_{1}, \ldots, x_{n}$ of the variable $x$, we define $p^{(n)}\left(x_{1}, \ldots, x_{n}\right)$ inductively by: $p_{1}\left(x_{1}\right)=p\left(x_{1}\right)$ and $p_{n}\left(x_{1}, \ldots, x_{n}\right)=p\left(x_{n}\right) \otimes$ $p_{n-1}\left(x_{1}, \ldots, x_{n-1}\right)$. We let $p^{(\omega)}\left(x_{1}, x_{2}, \ldots\right)$ be the union of the $p_{n}\left(x_{1}, \ldots, x_{n}\right)$, which will be a complete infinitary type over $\bar{M}$.

Assuming that $p(x) \in S(\bar{M})$ is $A$-invariant, by a Morley sequence in $p$ over $A$ we mean a realization $\left(a_{1}, a_{2}, a_{3}, \ldots\right)$ in $\bar{M}$ of $p^{(\omega)} \mid A$.

Lemma 2.3. Let $p(x) \in S(\bar{M})$ be invariant. Then:

(i) Any realization $\left(b_{1}, b_{2}, \ldots\right)$ of $p^{(\omega)}$ (in an elementary extension of $\bar{M}$ ) is an indiscernible sequence over $\bar{M}$.

(ii) Suppose $A \subset \bar{M}$ is small and $p$ is $A$-invariant. If $a_{1}, a_{2}, \ldots$ from $\bar{M}$ are such that $a_{n+1}$ realizes $p \mid\left(A a_{1}, \ldots, a_{n}\right)$, then $\left(a_{1}, a_{2}, \ldots\right)$ is a Morley sequence in $p$ over $A$. In particular $\operatorname{tp}\left(a_{1}, a_{2}, \ldots / A\right)$ depends only on $p$ and $A$.

Proof. Straightforward and left to the reader.

Remark 2.4. (Assume NIP.) More generally we can define a Morley sequence of $p \in$ $S(\bar{M})$ over $A$, assuming just that $p$ does not fork over $A$, to be a realization in $\bar{M}$ of $\operatorname{Lstp}\left(b_{1}, b_{2}, \ldots / A\right)$ where $\left(b_{1}, b_{2}, \ldots\right)$ realizes $p^{(\omega)}$ (in a model containing $\bar{M}$ ). This is consistent with the previous definition.

Lemma 2.5. (Assume NIP.)

(i) Suppose $p(x), q(x)$ are global types such that $p$ is A-invariant and $q$ does not fork over A. Then (a), (b), (c) below are equivalent:

(a) $p=q$.

(b) $p^{(\omega)}\left|A=q^{(\omega)}\right| A$.

(c) For all $n$ and realization e of $p^{(n)}|A, p| A e=q \mid A e$.

(ii) Suppose $Q\left(x_{0}, x_{1}, \ldots\right)$ is the type over A of some A-indiscernible sequence. Then $Q=p^{(\omega)} \mid$ A for some A-invariant global type $p(x)$ if and only if whenever $I_{j}$ for $j \in J$ are realizations of $Q$ then there is an element $c$ such that $\left(I_{j}, c\right)$ is $A$-indiscernible for all $j \in J$.

Proof. (i) Clearly (a) implies each of (b) and (c). Now let us see that (b) implies (c): First note that as $p$ is $A$-invariant, so is $p^{(\omega)}$, and hence $p^{(\omega)} \mid A$ implies $p^{(\omega)} \mid \operatorname{bdd}(A)$, so assuming (b) we have

(*) $p^{(n)}\left|\operatorname{bdd}(A)=q^{(n)}\right| \operatorname{bdd}(A)$ for all $n$.

Now let $e$ realize $p^{(n)} \mid A$, so $e$ realizes $p^{(n)} \mid \operatorname{bdd}(A)$, and so also $q^{(n)} \mid \operatorname{bdd}(A)$. For $a$ realizing $p \mid(\operatorname{bdd}(A) e),(e, a)$ realizes $p^{(n+1)} \mid \operatorname{bdd}(A)$, and likewise for $b$ realizing $q \mid(\operatorname{bdd}(A) e)$, 
$(e, b)$ realizes $q^{(n+1)} \mid \operatorname{bdd}(A)$. By $(*)$ for $n+1$, it follows that $p|(\operatorname{bdd}(A) e)=q|(\operatorname{bdd}(A) e)$ and in particular $p|A e=q| A e$. So we have shown that (b) implies (c).

So it suffices to prove that (c) implies (a), that is, if $p|A e=q| A e$ for any realization $e$ of any $p^{(n)} \mid A$, then $p=q$. Supposing for a contradiction that $p \neq q$ there is $\phi(x, b) \in p, \neg \phi(x, b) \in q$. Let $a_{1}, a_{2}, \ldots$ in $\bar{M}$ be such that $a_{i}$ realizes $p \mid\left(A a_{1} \ldots a_{i-1} b\right)$ for $i$ odd, and $a_{i}$ realizes $q \mid\left(A a_{1} \ldots a_{i-1} b\right)$ for $i$ even. Our assumption, together with Lemma 2.3(ii), implies that $\left(a_{1}, a_{2}, \ldots\right)$ is a Morley sequence in $p$ over $A$, hence by 2.3(i) indiscernible over $A$. But $\phi\left(a_{i}, b\right)$ holds iff $i$ is odd, contradicting Fact 1.1.

(ii) Left implies right is clear and does not require NIP. (Let $c$ realize $p \mid\left(A \cup \bigcup_{j} I_{j}\right)$.) For the other direction, assume $Q$ has the given property. Define the global type $p$ by: $\phi(x, b) \in p$ iff any realization $I$ of $Q$ extends to an $A$-indiscernible sequence $I^{\prime}$ such that $\phi(x, b)$ is eventually true on $I^{\prime}$. Then NIP and our assumptions on $Q$ show that $p$ is consistent, complete and $A$-invariant and that $Q$ is the type over $A$ of its Morley sequence.

We continue with some newer material. We first give a rather better and more general result on "Borel definability" than that in [16]. Given a (small) subset $A$ of $\bar{M}$, by a closed set over $A$ we mean the set of realizations in $\bar{M}$ of a partial type over $A$. An open set over $A$ is the complement (in the relevant ambient sort) of a closed set over $A$. From these we build in the usual way the Borel sets over $A$. Alternatively these correspond to the Borel subsets of the relevant Stone space of complete types over $A$. A global type $p(x)$ will be called Borel definable over $A$ if for any $L$-formula $\phi(x, y)$, the set of $b$ in $\bar{M}$ such that $\phi(x, b) \in p(x)$ is a Borel set over $A$. So if $p(x)$ is definable over $A$ in the usual sense then $p$ will be Borel definable over $A$, and if $p$ is Borel definable over $A$ then $p$ is $A$-invariant. In fact, we will be proving strong Borel definability over $A$, in the sense that for any $\phi(x, y) \in L$, the set of $b$ such that $\phi(x, b) \in p$ is a finite Boolean combination of closed sets over $A$.

Proposition 2.6. (Assume NIP.) Suppose that $p(x) \in S(\bar{M})$ is a global type which is $A$-invariant. Then $p$ is strongly Borel definable over A.

Proof. Let $\phi(x, y) \in L$. Let $N<\omega$ be as given for $\phi(x, y)$ by Fact 1.1 .

Claim. For any $b, \phi(x, b) \in p$ if and only if for some $n \leq N$ there is $\left(a_{1}, \ldots, a_{n}\right)$ realising $p^{(n)} \mid$ A such that

$(*)_{n} \models \phi\left(a_{i}, b\right) \leftrightarrow \neg \phi\left(a_{i+1}, b\right)$ for $i=1, \ldots, n-1$,

and $\models \phi\left(a_{n}, b\right)$, but there is no $\left(a_{1}, \ldots, a_{n+1}\right)$ realizing $p^{(n+1)} \mid A$ such that $(*)_{n+1}$ holds].

Proof of Claim. Suppose $\phi(x, b) \in p$. By Fact 1.1 choose any realization $\left(c_{i}: i<\omega\right)$ of $p^{(\omega)} \mid A$ with a maximal finite alternation (at most $N$ where $N$ is given for $\phi(x, y)$ by 1.1) of truth values of $\phi\left(c_{i}, b\right)$ for $i<\omega$. Hence, eventually $\phi\left(c_{i}, b\right)$ holds: for if not, let $c_{\omega}$ realize $p \mid\left(A \cup\left\{c_{i}: i<\omega\right\} \cup\{b\}\right)$, and we contradict maximality.

The converse holds by the above proof applied to $\neg \phi(x, b)$. So the claim is proved and clearly yields a strongly Borel definition of the set of $b$ such that $\phi(x, b) \in p$. 
Remark 2.7. (i) Define an $A$-invariant global type $p(x)$ to have NIP if its Morley sequence over $A,\left(b_{i}: i<\omega\right)$ (which has a unique type over $A$ ), has the property that for every $\phi(x, y) \in L$ there is $n_{\phi}<\omega$ such that for any $c$, there are at most $n_{\phi}$ alternations of truth values of $\phi\left(b_{i}, c\right)$. Then Proposition 2.6 goes through for $p$.

(ii) Proposition 2.6 also holds when $A$ is a set of hyperimaginaries, such as $\operatorname{bdd}(B)$ for some set $B$ of imaginaries.

We now consider (assuming NIP still) global types which do not fork over $A$, and Lascar strong types over $A$.

Remark 2.8. (Assume NIP.) Let $p, q$ be global types which do not fork over $A$. Then $p \otimes q$ does not fork over $A$. In particular $p^{(n)}$ and $p^{(\omega)}$ do not fork over $A$.

Proof. This follows from the well-known fact (valid for any theory $T$ ) that if $\operatorname{tp}(a / B)$ does not fork over $A$ and $\operatorname{tp}(b / B a)$ does not fork over $A a$ then $\operatorname{tp}(a, b / B)$ does not fork over $A$. We will give a quick proof of this fact for completeness:

First, using the hypotheses, find a saturated model $M$ containing $B$ such that $\operatorname{tp}(a / M)$ does not fork over $A$, and $\operatorname{tp}(b / M a)$ does not fork over $A a$. It is enough to prove:

Claim. $\operatorname{tp}(a b / M)$ does not divide over $A$.

Proof of Claim. Let $c_{0} \in M$, and $q\left(x, y, c_{0}\right)=\operatorname{tp}\left(a b / A c_{0}\right)$. Let $\left(c_{i}: i<\omega\right)$ be an $A$-indiscernible sequence. We must show that $\bigcup\left\{q\left(x, y, c_{i}\right): i<\omega\right\}$ is consistent. Let $p\left(x, c_{0}\right)=\operatorname{tp}\left(a / A c_{0}\right)$. As $\operatorname{tp}(a / M)$ does not fork over $A, \bigcup\left\{p\left(x, c_{i}\right): i<\omega\right\}$ is consistent, and we may assume (after applying Ramsey's theorem and some automorphisms) that $\left(c_{i}: i<\omega\right)$ is $A a$-indiscernible. But now, as $\operatorname{tp}(b / M a)$ does not fork over $A a$, we may find $b^{\prime}$ realizing $\bigcup\left\{q\left(a, y, c_{i}\right): i<\omega\right\}$. So then $\left(a, b^{\prime}\right)$ realizes $\bigcup\left\{q\left(x, y, c_{i}\right): i<\omega\right\}$, and we are finished.

Lemma 2.9. (Assume NIP.) Suppose that $p(x)$ is a global type which does not fork over A. Let $c, d$ realize $p \mid A$. Then $\operatorname{Lstp}(c / A)=\operatorname{Lstp}(d / A)$ iff there is an (infinite) sequence a such that both $(c, a)$ and $(d, a)$ realize $p^{(\omega)} \mid A$.

Proof. Right implies left is immediate (for, as remarked earlier, elements of an infinite $A$-indiscernible sequence have the same Lascar strong type over $A$ ).

Left to right: Note first that for some $\sigma \in \operatorname{Aut}(\bar{M} / A), \sigma(p)(x)$ implies $\operatorname{Lstp}(c / A)$. As $\sigma(p)^{(\omega)}\left|A=p^{(\omega)}\right| A$, we may assume that already $p(x) \operatorname{implies} \operatorname{Lstp}(c / A)$. Let $\left(a_{0}, a_{1}, a_{2}, \ldots\right)$ realize $p^{(\omega)}$ (in a bigger saturated model). So $\left(a_{1}, a_{2}, \ldots\right)$ also realizes $p^{(\omega)}$ and does not fork over $A$. Hence by 2.1 whether or not $\phi(\bar{x}, c)$ is in $p^{(\omega)}$ depends on $\operatorname{Lstp}(c / A)$. Hence $\operatorname{tp}\left(c, a_{1}, a_{2}, \ldots / A\right)=\operatorname{tp}\left(a_{0}, a_{1}, \ldots / A\right)=\operatorname{tp}\left(d, a_{1}, a_{2}, \ldots / A\right)=$ $p^{(\omega)} \mid A$, as required.

Lemma 2.9 says that on realizations of $p \mid A$, having the same Lascar strong type over $A$ is a type-definable (over $A$ ) equivalence relation, hence by our discussion of Lascar strong types in the introduction we see: 
Corollary 2.10. (Assume NIP.)

(i) Suppose $p(x) \in S(A)$ does not fork over A. Then on realisations of $p$, Lascar strong type over A coincides with compact (KP) strong type over A.

(ii) Suppose that any complete 1-type over any set A does not fork over A. Then over any set A, Lascar strong types coincide with compact strong types, that is, $T$ is " $G$ compact" over any set of parameters.

Proof. (ii) The assumption, together with the discussion in the proof of 2.8, implies that any complete type over any set $A$ does not fork over $A$. So we can apply (i).

We can now strengthen Proposition 2.1.

Proposition 2.11. (Assume NIP.) Suppose that $p(x)$ is a global type. Then $p$ does not fork over $A$ if and only if $p$ is $\operatorname{bdd}(A)$-invariant.

Proof. Right to left is clear and does not use the NIP assumption. For left to right, assume $p$ does not fork over $A$. Let $\sigma$ be an automorphism of $\bar{M}$ fixing bdd $(A)$ pointwise, and we have to show that $\sigma(p)=p$. By Remark 2.8 and Corollary 2.10(i) we have

Claim I. For any realization $\bar{a}$ of $p^{(\omega)} \mid A, \operatorname{Lstp}(\bar{a} / A)=\operatorname{Lstp}(\sigma(\bar{a}) / A)$.

By Claim I and Proposition 2.1(i) it follows that

Claim II. For any realization $\bar{a}$ of $p^{(\omega)}|A, p| A \bar{a}=\sigma(p) \mid A \bar{a}$.

Now let $M$ be a small model containing $A$, and let $\bar{a}$ realize $p^{(\omega)} \mid M$.

Claim III. For any $c$ realizing either $p \mid A \bar{a}$ or $\sigma(p) \mid A \bar{a}$, $\bar{a} c$ is an A-indiscernible sequence.

Proof of Claim III. By Claim II, it is enough to prove that $\bar{a} c$ is $A$-indiscernible for $c$ realizing $p \mid A \bar{a}$. Note that $p$ does not fork over $M$, hence by 2.1(i) is $M$-invariant. So by Lemma 2.3, if $c$ realizes $p \mid M \bar{a}$ then $\bar{a} c$ is $M$-indiscernible, hence also $A$-indiscernible. But $c$ realizes $p \mid A \bar{a}$ and the latter is a complete type over $A \bar{a}$. So for any $c$ realizing $p \mid A \bar{a}$, $\bar{a} c$ is an $A$-indiscernible sequence.

Claim IV. Let $\bar{a}^{\prime}$ be an indiscernible sequence (of realizations of $p \mid A$ ) extending $\bar{a}$. Then for c realizing either $p \mid A \bar{a}^{\prime}$ or $\sigma(p) \mid A \bar{a}^{\prime}, \bar{a}^{\prime} c$ is A-indiscernible.

Proof of Claim IV. This can be seen in various ways. For example it can be deduced from Claim III, using the fact that each of $p, \sigma(p)$ is invariant under $\operatorname{Autf}(\bar{M} / A)$, and the fact that any two increasing $n$-tuples from $\bar{a}^{\prime}$ have the same Lascar strong type over $A$.

Now suppose for a contradiction that $p \neq \sigma(p)$. So for some $\psi(x, y) \in L$ and $e \in \bar{M}$, $\psi(x, e) \in p$ and $\neg \psi(x, e) \in \sigma(p)$. Let $c_{i}$ realize $p \mid A \bar{a} c_{0} \ldots c_{i-1} e$ for $i$ even and realize $\sigma(p) \mid A \bar{a} c_{0} \ldots c_{i-1} e$ for $i$ odd. By Claim IV, $\left(\bar{a}, c_{i}\right)_{i}$ is $A$ indiscernible. But $\models \psi\left(c_{i}, e\right)$ iff $i$ is even, contradicting NIP. This concludes the proof of Proposition 2.11.

Finally we will give an analogue of 2.10(ii) for strong types (which is closely related to material in [19] and [18]). First a preparatory lemma. 
Lemma 2.12. (Assume NIP.) Suppose A is algebraically closed, $\operatorname{tp}(a / A)$ has a global Ainvariant extension, and $e \in \operatorname{acl}(A a)$. Then $\operatorname{tp}(a e / A)$ has a global A-invariant extension.

Proof. Let $p(x)=\operatorname{tp}(a / A)$ and fix some global $A$-invariant (so nonforking) extension $p^{\prime}(x)$ of $p(x)$. Let $q(x, y)=\operatorname{tp}(a e / A)$, and let $q^{\prime}(x, y)$ be any global extension of $q(x, y)$ whose restriction to $x$ is $p^{\prime}(x)$. We will show that $q^{\prime}(x, y)$ is $A$-invariant. Let $\delta(x, y)$ be a formula over $A$ which is true of $(a, e)$ and such that for any $a^{\prime}, \delta\left(a^{\prime}, y\right)$ is algebraic (if consistent).

Claim I. $q^{\prime}$ does not fork over A.

Proof of Claim I. Let $\phi(x, y, b) \in q^{\prime}(x, y)$ and let $\left(b_{i}: i<\omega\right)$ be $A$-indiscernible with $b_{0}=b$. We may assume that $\models \phi(x, y, b) \rightarrow \delta(x, y)$. As $p^{\prime}$ does not fork over $A$, there is $a^{\prime}$ realizing $\left\{\exists y \phi\left(x, y, b_{i}\right): i<\omega\right\}$. For each $i$, let $e_{i}$ realize $\phi\left(a^{\prime}, y, b_{i}\right)$. As there are finitely many possible choices for the $e_{i}$, there is an infinite subset $I$ of $\omega$ such that $e_{i}=e_{j}$ for $i, j \in I$. So $\left\{\phi\left(x, y, b_{i}\right): i \in I\right\}$ is consistent, which is enough.

As $p^{\prime}$ is $A$-invariant so is $p^{(n)}$ for any $n$, we obtain:

Claim II. For all n, $p^{\prime(n)} \mid A$ implies a "complete" Lascar strong type over A.

Claim III. For any $n, q^{\prime(n)} \mid A$ implies a "complete" Lascar strong type over A.

Proof of Claim III. As $q^{\prime(n)}$ does not fork over $A$, by Lemma 2.9 the relation of having the same Lascar strong type over $A$ on realizations of $q^{\prime(n)} \mid A$ is type-definable over $A$. But by Claim II clearly there are only finitely many Lascar strong types over $A$ extending $q^{\prime(n)} \mid A$ : If $\left(\left(a_{1}, e_{1}\right), \ldots,\left(a_{n}, e_{n}\right)\right)$, and $\left(\left(a_{1}^{\prime}, e_{1}^{\prime}\right), \ldots,\left(a_{n}^{\prime}, e_{n}^{\prime}\right)\right)$ are realizations of $q^{\prime(n)} \mid A$ with distinct Lascar strong types, we may by Claim II assume that $a_{i}=a_{i}^{\prime}$ for $i=1, \ldots, n$, so there are only finitely many possibilities for the sequence $\left(e_{i}\right)_{i}$. Hence equality of Lascar strong type on realizations of $q^{\prime(n)} \mid A$ is the restriction to $q^{\prime(n)} \mid A$ of a finite $A$-definable equivalence relation. As $A$ is algebraically closed, there is just one Lascar strong type over $A$ extending $q^{\prime(n)}$, proving Claim III.

The $A$-invariance of $q^{\prime}$ follows from Claim III, as in the proof of 2.11 .

Proposition 2.13. (Assume NIP.) Let $T$ be 1-sorted and work in $T^{\mathrm{eq}}$. The following are equivalent:

(i) For any algebraically closed set $A$ and complete 1-type $p$ over $A$ in the home sort, $p$ has a global A-invariant extension.

(ii) For any complete type $p$ over any algebraically closed set A, $p$ has a global Ainvariant extension.

(iii) For any $A$, (a) any $p(x) \in S(A)$ does not fork over $A$, and (b) Lascar strong types over A coincide with strong types over A.

Proof. (i) implies (ii). It is enough to prove that for any $n$ any complete $n$-type (in the home sort) over any algebraically closed set $A$ has a global $A$-invariant extension. We prove it by induction on $n$. Suppose it is true for $n$. Let $p\left(x_{1}, \ldots, x_{n}, x_{n+1}\right)=$ $\operatorname{tp}\left(a_{1}, \ldots, a_{n}, a_{n+1} / A\right)$ with the $a_{i}$ 's elements of the home sort, and $A$ algebraically 
closed. Let (the infinite tuple) $e$ be an enumeration of $\operatorname{acl}\left(A a_{1}, \ldots, a_{n}\right)$. By the induction hypothesis and $2.12, \operatorname{tp}(e / A)$ has a global $A$-invariant extension, realized by $e^{\prime}$ say (in a bigger saturated model $\bar{M}^{\prime}$ ). Let $a_{1}^{\prime}, \ldots, a_{n}^{\prime}$ denote the copies of the $a_{i}$ in $e^{\prime}$. By the hypothesis over the algebraically closed base $e^{\prime}$, there is $a_{n+1}^{\prime}$ (in the bigger model) with $\operatorname{tp}\left(e^{\prime}, a_{n+1}^{\prime} / A\right)=\operatorname{tp}\left(e, a_{n+1} / A\right)$ such that $\operatorname{tp}\left(a_{n+1}^{\prime} / \bar{M} e^{\prime}\right)$ does not split over $e^{\prime}$. It follows easily that $\operatorname{tp}\left(e^{\prime}, a_{n+1}^{\prime} / \bar{M}\right)$ is $A$-invariant, hence also $\operatorname{tp}\left(a_{1}^{\prime}, \ldots, a_{n}^{\prime}, a_{n+1}^{\prime} / \bar{M}\right)$ is the $A$-invariant extension of $p$ we are looking for.

(ii) implies (iii) and (iii) implies (i) are clear.

Corollary 2.14. If $T$ is o-minimal or C-minimal then (i) to (iii) of Proposition 2.13 hold.

Proof. Condition (i) holds in $C$-minimal theories through the existence of "generic" 1 types (see [11]). In the $o$-minimal case, (i) holds without even the condition that $A$ is algebraically closed.

\section{Generically stable types}

Here we make a systematic study of what Shelah has called "stable types" in [35]. We discuss our choice of language a bit later. We begin with some preliminary remarks.

Lemma 3.1. (Assume NIP.) Let $p(x)$ be a global type which does not fork over a small set $A$.

(i) Suppose $p$ is definable. Then $p$ is definable over $\operatorname{acl}(A)$. In particular $p$ is $\operatorname{acl}(A)$ invariant.

(ii) Suppose that $p$ is finitely satisfiable in some small model. Then $p$ is finitely satisfiable in any model which contains $A$.

Proof. (i) By 2.11, $p$ is $\operatorname{bdd}(A)$-invariant. So if $p$ is definable, then for any $\phi(x, y) \in L$ the $\phi$-definition of $p$ is over $\operatorname{bdd}(A)$, hence over $\operatorname{acl}(A)$.

(ii) Let $M_{1}$ be a small model in which $p$ is finitely satisfiable. Let $M$ be an arbitrary (small) model containing $A$. Let $\phi(x, c) \in p$. Let $M_{1}^{\prime}$ realize a coheir of $\operatorname{tp}\left(M_{1} / M\right)$ over $M c$. As $p$ is $M$-invariant, $p$ is finitely satisfiable in $M_{1}^{\prime}$ so there is $a^{\prime} \in M_{1}^{\prime}$ such that $\models \phi(a, c)$. So there is $a^{\prime} \in M$ such that $\models \phi(a, c)$.

Among our main results is:

Proposition 3.2. (Assume NIP.) Let $p(x) \in S(\bar{M})$, and let $A$ be such that $p$ is $A$ invariant. Then the following conditions are equivalent:

(i) $p(x)$ is definable (hence A-definable), and also finitely satisfiable in somelany small model containing $A$.

(ii) $p^{(\omega)} \mid A$ is totally indiscernible. That is, if $\left(a_{i}: i<\omega\right)$ is a Morley sequence in $p$ over $A$, then $\left(a_{i}: i<\omega\right)$ is an indiscernible set (not just sequence) over $A$.

(iii) For any formula $\phi(x, y)$ there is $N$ such that for any Morley sequence $\left(a_{i}: i<\omega\right)$ of $p$ over $A$, and any $c, \phi(x, c) \in p$ if and only if $\models \bigvee_{w \subset 2 N,|w|=N} \bigwedge_{i \in w} \phi\left(a_{i}, c\right)$.

(iv) For all small $B \supseteq A, p$ is the unique global nonforking extension of $p \mid B$. 
Moreover conditions (i)-(iv) imply:

(v) For all $n, p^{(n)}$ is the unique global nonforking extension of $p^{(n)} \mid A$.

Conversely, if A has the additional property that every complete type over A does not fork over A, then (v) implies each of (i)-(iv).

Proof. (i) implies (ii). Fix a small model $M \supseteq A$ such that $p$ is finitely satisfiable in $M$ (and of course definable over $M$ ), and there is no harm in proving (ii) with $M$ in place of $A$. Let $\left(a_{i}: i<\omega\right)$ be a Morley sequence in $p$ over $M$. We will show

(*) for any $n$ and $i \leq n, a_{i}$ realizes the restriction of $p$ to $M a_{0} \ldots a_{i-1} a_{i+1} \ldots a_{n}$.

Note that $(*)$ will be enough to show by induction that for any $n$ and permutation $\pi$ of $\{0, \ldots, n\}, \operatorname{tp}\left(a_{0}, \ldots, a_{n} / M\right)=\operatorname{tp}\left(a_{\pi(0)}, \ldots, a_{\pi(n)} / M\right)$ which will prove the total indiscernibility of $\left(a_{i}: i<\omega\right)$.

To prove $(*)$, note that $\operatorname{tp}\left(a_{i+1}, \ldots, a_{n} / M a_{0}, \ldots, a_{i}\right)$ is finitely satisfiable in $M$. As $\operatorname{tp}\left(a_{i} / a_{0}, \ldots, a_{i-1}, M\right)$ is definable over $M$, it follows that $\operatorname{tp}\left(a_{0} \ldots a_{i-1} a_{i+1} \ldots a_{n} / M a_{i}\right)$ is finitely satisfiable in $M$, whence $\operatorname{tp}\left(a_{i} / M a_{0} \ldots a_{i-1} a_{i+1} \ldots a_{n}\right)$ is an heir of $p \mid M$, so (as $p$ is definable), equals $p \mid\left(M a_{0} \ldots a_{i-1} a_{i+1} \ldots a_{n}\right)$. This proves $(*)$.

(ii) implies (iii). This is by the "in particular" clause of Fact 1.1.

(iii) implies (i). Clearly $p$ is definable. But it also follows from (iii) that $p$ is finitely satisfiable in any model $M$ containing $A$. For suppose $\phi(x, c) \in p$. Let $I$ be a Morley sequence in $p$ over $A$ such that $\operatorname{tp}(I / M c)$ is finitely satisfiable in $M$. By (iii), $\phi(a, c)$ for some $a \in I$, hence $\phi(a, c)$ for some $a \in M$.

(ii) implies (v). As (ii) for $p$ clearly implies (ii) for $p^{(n)}$, it suffices to prove (v) for $n=1$, namely that $p$ is the unique global nonforking extension of $p \mid A$.

Let $q$ be an arbitrary global nonforking extension of $p \mid A$. Let $I=\left(a_{i}: i<\omega\right)$ be a Morley sequence in $p$ over $A$. We will prove that $I$ realizes $q^{(\omega)} \mid A$, which will be enough, by Lemma 2.5, to conclude that $p=q$. So let $b$ realize $q \mid(A \cup I)$. We prove inductively that

(**) $\operatorname{tp}\left(a_{0}, \ldots, a_{n}, b / A\right)=\operatorname{tp}\left(a_{0}, \ldots, a_{n}, a_{n+1} / A\right)$ for all $n$.

Note that of course $\operatorname{tp}\left(a_{0} / A\right)=\operatorname{tp}(b / A)=p \mid A$, which is in a sense the pre-base step.

So assume $(* *)$ is true for $n-1$. Suppose that $\models \phi\left(a_{0}, \ldots, a_{n}, b\right)$. As $q$ does not fork over $A$, we find, by 2.1 and indiscernibility of $I$, that $\models \phi\left(a_{0}, \ldots, a_{n-1}, a_{i}, b\right)$ for all $i \geq n$. By (iii) (for $p$ ) we see that $\phi\left(a_{0}, \ldots, a_{n-1}, x, b\right) \in p(x)$. By the induction hypothesis and the $A$-invariance of $p$, we conclude that $\phi\left(a_{0}, \ldots, a_{n-1}, x, a_{n}\right) \in p$, and therefore $\models \phi\left(a_{0}, \ldots, a_{n-1}, a_{n+1}, a_{n}\right)$. Finally total indiscernibility of $I$ implies that $\models \phi\left(a_{0}, \ldots, a_{n-1}, a_{n}, a_{n+1}\right)$ as required.

As condition (i) is preserved after replacing $A$ by any $B \supseteq A$, it follows from what we have proved up to now that each of (i), (ii), (iii) implies (iv).

(iv) implies (iii). Let $I=\left(a_{i}: i<\omega\right)$ be any Morley sequence in $p$ over $A$. Note that $\operatorname{Av}(I / \bar{M})$ is an $A \cup I$-invariant extension of $p \mid A I$, hence equals $p$. It follows easily that for each $\phi(x, y)$ there is $N$ such that for any $c$ either $\left|\left\{i<\omega: \models \phi\left(a_{i}, c\right)\right\}\right|<N$ or $\left|\left\{i<\omega: \models \neg \phi\left(a_{i}, c\right)\right\}\right|<N$. So we obtain (iii). 
Now assume the additional hypothesis on $A$, and we prove

(v) implies (ii). With some abuse of notation, let $p^{\left(\omega^{*}\right)}\left(x_{0}, x_{1}, \ldots\right)$ denote $\operatorname{tp}\left(\left(a_{0}, a_{1}, a_{2} \ldots\right) / \bar{M}\right)$, where for each $n,\left(a_{n-1}, a_{n-2}, \ldots, a_{0}\right)$ realizes $p^{(n)}$. Let $Q\left(x_{i}\right)_{i \in \omega}$ be the restriction of $p^{\left(\omega^{*}\right)}$ to $A$. So (v) implies that

$(* * *) p^{\left(\omega^{*}\right)}$ is the unique global nonforking extension of $Q$.

Note that if $a$ realizes $p \mid A$ and $\left(a_{i}\right)_{i}$ realizes the restriction of $p^{\left(\omega^{*}\right)}$ to $A a$ then $\left(a_{0}, a_{1}, \ldots, a_{n}, \ldots, a\right)$ is $A$-indiscernible. We claim that $Q$ satisfies the right hand side condition of 2.5(ii), namely whenever $I_{1}, I_{2}$ are realizations of $Q$ then there is $c$ such that $\left(I_{j}, c\right)$ is $A$-indiscernible, for $j=1,2$. For, by our hypothesis on $A$, let $I_{1}^{\prime}, I_{2}^{\prime}$ realize a global nonforking extension of $\operatorname{tp}\left(I_{1}, I_{2} / A\right)$. By $(* * *)$, each of $I_{1}^{\prime}, I_{2}^{\prime}$ realizes $p^{\left(\omega^{*}\right)}$. So choosing $c \in \bar{M}$ realizing $p \mid A$, realizing in $\bar{M}$ the restriction of $\operatorname{tp}\left(I_{1}^{\prime}, I_{2}^{\prime} / \bar{M}\right)$ to $A c$, and using an automorphism, gives the claim. By 2.5(ii), $Q=q^{(\omega)} \mid A$ for some $A$-invariant global type $q$, which must extend $p \mid A$. Hence, by $(\mathrm{v}), q=p$. We have shown that $\operatorname{tp}\left(a_{0}, a_{1}, \ldots, a_{n-1} / A\right)=\operatorname{tp}\left(a_{n-1}, a_{n-2}, \ldots, a_{0} / A\right)$ whenever $\left(a_{0}, \ldots, a_{n-1}\right)$ realizes $p^{(n)} \mid A$. Total indiscernibility of (a realization of) $p^{(\omega)} \mid A$ follows easily, using $A$ invariance of $p$. So we have proved (ii). The proof of Proposition 3.2 is complete.

Remark 3.3. (i) Assuming NIP, we will call a global type $p(x)$ generically stable if it is both definable and finitely satisfiable in some small model, that is, $p$ satisfies 3.2(i) for some $A$. We may also want to talk about generically stable types without a NIP assumption, in which case we will mean a global $p$ such that for some $A$, (i)-(iv) of 3.2 are satisfied. We leave it to the reader to study the implications between (i)-(iv) in the absence of NIP.

(ii) (Assume NIP.) Suppose the global type $p$ is generically stable, and $p$ does not fork over $B$. Then by 3.1, $p$ satisfies 3.2(i) with $A=\operatorname{acl}(B)$. Hence by Proposition 3.2, we recover the finite equivalence relation theorem: any two global nonforking extensions of $p \mid B$ are distinguished by some finite $B$-definable equivalence relation.

(iii) Proposition 3.2 goes through assuming only that $p$ has NIP (as in Remark 2.7).

(iv) In $A C V F$, for any set $A$, any complete type over $A$ has a global nonforking extension. (See [11] or Proposition 2.13.) Hence (i)-(v) of 3.2 are equivalent in $A C V F$, for any $A$.

(v) In 3.2(iv) it is not enough to require just that $p \mid A$ has a unique global nonforking extension.

Explanation of $(v)$. So we give an example of a NIP theory and a type $p(x) \in S(A)$ with a unique global $A$-invariant extension which is not definable. We consider the basic $C$ minimal theory consisting of a dense linear ordering $(I,<)$ with greatest element $\infty$, and another sort on which there are equivalence relations $E_{i}$ indexed (uniformly) by $i \in I$ and with $E_{j}$ infinitely refining $E_{i}$ if $i<j$ (plus some other axioms, see [15]). By a definable ball we mean an $E_{i}$-class for some $E_{i}$. By a type-definable ball we mean a possibly infinite intersection of definable balls. We can produce a model $M$ and a type-definable (over $M$ ) ball $B$ such that $B$ contains no proper $M$-definable ball. Let the global type $p_{B}(x) \in S(\bar{M})$ be the "generic type" of $B$, that is, $p$ says that $x \in B$ and $x$ is not in any 
proper definable sub-ball of $B$. Then $p$ is the unique $M$-invariant extension of $p \mid M$ but is not definable.

In the NIP context, our generically stable types coincide with what Shelah [35] calls stable types. However there is already another meaning for a complete or even partial type $\Sigma(x)$ to be stable. It is that any extension of $\Sigma(x)$ to a complete global type is definable (over some set, not necessarily the domain of $\Sigma$ ). This notion is also sometimes called "stable, stably embedded" (although mainly in the case where the partial type is a single formula). One family of examples of generically stable types come through stable domination in the sense of [11]. Recall that $p(x) \in S(A)$ ( $A$ algebraically closed) is said to be stably dominated if there is a stable partial type $\Sigma(x)$ over $A$ (stable in the strong sense mentioned above), and an $A$-definable function $f$ from the set of realizations of $p$ to the realizations of $\Sigma$ such that, if $q \in S(A)$ is $f(p)$, and $a$ realizes $p$, then whenever $f(a)$ is independent of $B$ over $A$ then $\operatorname{tp}(a / A, f(a))$ has a unique extension over $B f(a)$. A stably dominated type is generically stable, as is easily verifiable. Generically stable but unstable types occur in algebraically closed valued fields through stable domination, namely via the stable part of the structure which is essentially the residue field. In "pure" unstable $N I P$ theories such as $o$-minimal and weakly $o$-minimal theories, or $p$-adically closed fields there are no (nonalgebraic) generically stable types. On the other hand in simple theories any stationary type (type with a unique nonforking extension) is easily seen to be generically stable.

We finish this section with some remarks on invariant types and symmetry.

Lemma 3.4. Suppose $p(x), q(y)$ are global types such that $p$ is finitely satisfiable in some small model, and $q$ is definable. (So both $p$ and $q$ are invariant.) Then $p_{x} \otimes q_{y}=$ $q_{y} \otimes p_{x}$.

Proof. Let $M$ be a small model such that $q$ is definable over $M$ and $p$ is finitely satisfiable in $M$. Let $(a, b)$ realize $(p(x) \otimes q(y)) \mid M$, that is, $b$ realizes $q \mid M$ and $a$ realizes $p \mid M b$. We want to show that $(a, b)$ realizes $(q(y) \otimes p(x)) \mid M$. Suppose not. Then there is a formula $\phi(a, y) \in q$ such that $\models \neg \phi(a, b)$. Let $\psi(x)$ (a formula over $M$ ) be the $\phi(x, y)$-definition for $q(y)$. So $\models \psi(a)$. As $\operatorname{tp}(a / M b)$ is finitely satisfiable in $M$, there is $a^{\prime} \in M$ such that $\models \psi\left(a^{\prime}\right) \wedge \neg \phi\left(a^{\prime}, b\right)$, which is a contradiction as $\operatorname{tp}(b / M)=q \mid M$. (The reader should note that this is just a restatement of uniqueness of heirs for definable types.)

Finally we return to generically stable types:

Proposition 3.5. Suppose $T$ has NIP, and that $p(x), q(y)$ are global invariant types such that $p(x)$ is generically stable. Then $p_{x} \otimes q_{y}=q_{y} \otimes p_{x}$.

Proof. Suppose $\phi(x, y) \in L_{\bar{M}}$ and $\phi(x, y) \in p(x) \otimes q(y)$. Let $M$ be a small model such that neither $p$ nor $q$ forks over $M$ and $\phi$ is over $M$. By assumption, $p$ is definable over $M$. Moreover

(*) if $\psi(y)$ is the $\phi(x, y)$-definition of $p$, then for any realization $c$ of $\psi$, and any Morley sequence $\left(a_{i}: i<\omega\right)$ of $p$ over $M, \models \phi\left(a_{i}, c\right)$ for all but finitely many $i<\omega$. 
From our assumption that $\phi(x, y) \in p(x) \otimes q(y)$, it follows that $\psi(y) \in q$. Now suppose for a contradiction that $\neg \phi(x, y) \in q(y) \otimes p(x)$. Let $\left(a_{i}: i<\omega\right)$ be a Morley sequence in $p$ over $M$. Then $\neg \phi\left(a_{i}, y\right) \in q$ for all $i$, so there is $b$ realizing $q \mid\left(M \cup\left\{a_{i}: i<\omega\right\}\right)$ such that $\models \neg \phi\left(a_{i}, b\right)$ for all $i$. As $b$ realizes $\psi(y)$ this contradicts $(*)$, and completes the proof.

\section{Measures and forking}

In this section we generalize some of the results on types in the previous sections to Keisler measures.

Recall that a Keisler measure $\mu$ on a sort $S$ over a set of parameters $A$ is a finitely additive probability measure on $A$-definable subsets of $S$ (or on formulas over $A$ with free variable in sort $S)$, that is, $\mu(X) \in[0,1]$ for all $A$-definable $X, \mu(S)=1$ and the measure of the union of two disjoint $A$-definable sets $X$ and $Y$ is the sum of the measures of $X$ and of $Y$. A complete type over $A$ (in sort $S$ ) can be identified with a Keisler measure on sort $S$ over $A$ with values in the set $\{0,1\}$. We sometimes write $\mu(x)$ or $\mu_{x}$ to mean that the measure is on the sort ranged over by the variable $x$. A Keisler measure over $\bar{M}$ is called a global (Keisler) measure. We have the obvious notion of a Keisler measure $\mu$ on sort $S$ over $B$ being an extension of a Keisler measure $\tau$ on sort $S$ over $A$ where $A \subseteq B$. In particular we can speak of a Keisler measure extending a type.

Let us emphasize that a Keisler measure $\mu_{x}$ over $A$ is the same thing as a ( $\sigma$-additive) regular Borel probability measure on the compact space $S_{x}(A)$. Regularity means that for any Borel subset $B$ of $S(A)$, and $\epsilon>0$, there are closed $C$ and open $U$ such that $C \subseteq B \subseteq U$ and $\mu(U \backslash C)<\epsilon$. Note that $\mu_{x}$ defines a finitely additive probability measure (still called $\mu$ ) on the algebra of clopens of $S(A)$. Theorem 1.2 of [20] extends $\mu$ to a Borel probability measure $\beta$ on $S_{x}(A)$ using the Loeb measure construction in a mild way. And Lemma 1.3(i) of [20] says that this $\beta$ is regular. On the other hand a Borel probability measure $\beta$ on $S_{x}(A)$ gives, by restriction to the clopens, a Keisler measure $\mu_{x}$ over $A$. Moreover if $\beta$ is regular then for any closed set $C, \beta(C)$ will be the infimum of the $\mu\left(C^{\prime}\right)$ where $C^{\prime}$ is a clopen containing $C$. Hence $\beta$ is determined by $\mu$.

An important, and even characteristic, fact about NIP theories is that for any global Keisler measure $\mu_{x}$ there are only boundedly many definable sets up to $\mu_{x}$-equivalence. (See Corollary 3.4 of [16].)

As in [16], notions relating to types generalize naturally to measures. There we discussed the notions of a measure being definable, and of being finitely satisfiable: For example if $\mu$ is a global Keisler measure then $\mu$ is definable over $A$ if for each closed subset $C$ of $[0,1]$ and $L$-formula $\phi(x, y),\{b \in \bar{M}: \mu(\phi(x, b)) \in C\}$ is type-definable over $A$. We say $\mu$ is finitely satisfiable in $M$ if every formula with positive measure is realized by a tuple from $M$. But we also have the notion of forking:

Definition 4.1. Let $\mu$ be a Keisler measure over $B$, and $A \subseteq B$. We say that $\mu$ does not divide over $A$ if whenever $\phi(x, b)$ is over $B$ and $\mu(\phi(x, b))>0$ then $\phi(x, b)$ does not divide over $A$. Similarly we say $\mu$ does not fork over $A$ if no formula of positive $\mu$-measure forks over $A$. 
Remark 4.2. Suppose $\mu$ is a global Keisler measure.

(i) $\mu$ does not fork over $A$ iff $\mu$ does not divide over $A$.

(ii) If $\mu$ is either definable over $M$ or finitely satisfiable in $M$, then $\mu$ does not fork over $M$.

Proof. (i) If $\mu(\phi(x))>0$ and $\phi$ forks over $A$ then $\phi$ implies a finite disjunction of formulas each of which divides over $A$. One of those formulas must have positive measure by finite additivity.

(ii) If $\mu$ is either definable over or finitely satisfiable in $M$, then for any $\phi(x, y) \in L$ and $b, \mu(\phi(x, b))$ depends on $\operatorname{tp}(b / M)$. (In the case that $\mu$ is definable over $M$ this is immediate. If $\mu$ is finitely satisfiable in $M$, then $\operatorname{tp}\left(b_{1} / M\right)=\operatorname{tp}\left(b_{2} / M\right)$ implies that the measure of the symmetric difference of $\phi\left(x, b_{1}\right)$ and $\phi\left(x, b_{2}\right)$ is 0 , so again $\phi\left(x, b_{1}\right)$ and $\phi\left(x, b_{2}\right)$ have the same measure.) So if $\left(b_{i}: i<\omega\right)$ is an $M$-indiscernible sequence and $\mu\left(\phi\left(x, b_{0}\right)\right)=r>0$, then for all $i, \mu\left(\phi\left(x, b_{i}\right)\right)=r$. By Lemma 2.8 of [16], $\left\{\phi\left(x, b_{i}\right): i<\omega\right\}$ is consistent.

Proposition 2.1 readily generalizes to measures.

Proposition 4.3. (Assume NIP.) Suppose $\mu$ is a global Keisler measure, and A a small set. Then the following are equivalent:

(i) $\mu$ does not fork over A.

(ii) $\mu$ is invariant under $\operatorname{Autf}(\bar{M} / A)$.

(iii) Whenever $\operatorname{Lstp}\left(b_{1} / A\right)=\operatorname{Lstp}\left(b_{2} / A\right)$, then $\mu\left(\phi\left(x, b_{1}\right) \Delta \phi\left(x, b_{2}\right)\right)=0$.

Proof. (i) implies (iii). Suppose that $\mu$ does not fork over $A$. Let $b_{0}, b_{1}$ begin an $A$ indiscernible sequence $\left(b_{i}: i<\omega\right)$. We claim that $\mu\left(\phi\left(x, b_{0}\right) \Delta \phi\left(x, b_{1}\right)\right)=0$. If not then without loss $\mu\left(\phi\left(x, b_{0}\right) \wedge \neg \phi\left(x, b_{1}\right)\right)>0$. As $\left(\left(b_{i}, b_{i+1}\right): i=0,2,4, \ldots\right)$ is $A$-indiscernible and $\mu$ does not fork over $A,\left\{\phi\left(x, b_{i}\right) \wedge \neg \phi\left(x, b_{i+1}\right): i=0,2, \ldots\right\}$ is consistent, contradicting NIP. So we clearly obtain (iii).

(iii) implies (ii) is immediate.

(ii) implies (i). Assume (ii) and suppose that $\mu(\phi(x, b))=\epsilon>0$ and that $(b=$ $\left.b_{0}, b_{1}, \ldots\right)$ is $A$-indiscernible. So the $b_{i}$ have the same Lascar strong type over $A$, hence $\mu\left(\phi\left(x, b_{i}\right)\right)=\epsilon$ for all $i$. By Lemma 2.8 of [16] again, $\left\{\phi\left(x, b_{i}\right): i<\omega\right\}$ is consistent.

So we see that the global Keisler measures which do not fork over some small set coincide with those which are invariant over some small model, and we can just call them invariant global measures (as we did for types). There is an obvious notion of Borel definability for a measure. Namely we say that (global) $\mu$ is Borel definable over $A$ if for any $\phi(x, y)$ and closed subset $C$ of $[0,1]$ the set of $b \in \bar{M}$ such that $\mu(\phi(x, b)) \in C$ is Borel over $A$. We will prove at the end of this section that (assuming NIP) any global invariant Keisler measure is Borel definable.

We can therefore define a product of invariant measures (global Keisler measures invariant over some small set or model) by integration: Suppose $\mu_{x}$ is a Borel definable (over $M$ ) global Keisler measure and $\lambda_{y}$ is another global Keisler measure. Fix $\phi(x, y) \in L_{\bar{M}}$. For any $b \in \bar{M}$ let $f(b)=\mu(\phi(x, b))$. Then $f$ is a Borel function 
over $M$ on the sort to which the $b$ 's belong, so we can form the integral $\int f(y) d \lambda_{y}$, and we call this $(\mu \otimes \lambda)(\phi(x, y))$. When $\mu, \lambda$ are global complete types, this agrees with the product as defined in Section 2.

Remark 4.4. It is natural to attempt to generalize the material from Section 3 to measures. So (assuming NIP) we call a global Keisler measure $\mu$ generically stable if $\mu$ is finitely satisfiable in some small model, and also definable. The analogues of Propositions 3.2 and Proposition 3.5 will be treated in a future work joint with P. Simon. Likewise for the measure analogue of the symmetry Lemma 3.4.

For now, we continue the generalization of the results from Section 2 to measures. We also begin to relate invariant measures (those measures which do not fork over a small set) to invariant types. We first generalize Proposition 2.11.

Proposition 4.5. (Assume NIP.) Let $\mu_{x}$ be a global Keisler measure which does not fork over $A$. Then $\mu$ is $\operatorname{bdd}(A)$-invariant.

Proof. Let $B$ be the Boolean algebra of formulas $\phi(x, b)$ over $\bar{M}$ quotiented by the equivalence relation $\phi(x, b) \sim \psi(x, c)$ if $\mu(\phi(x, b) \Delta \psi(x, c))=0$. Let $[\phi(x, b)]$ denote the class of $\phi(x, b)$, i.e. the image of $\phi(x, b)$ in $B$. Note that $B$ is small, because by Proposition 4.3,

$(*)$ if $\operatorname{Lstp}(b / A)=\operatorname{Lstp}(c / A)$ then $[\phi(x, b)]=[\phi(x, c)]$.

For any ultrafilter $U$ on $B$, we obtain a complete global type $p_{U}(x)$ by putting $\phi(x, b) \in$ $p_{U}$ iff $[\phi(x, b)] \in U$. Note that by $(*), p_{U}$ is invariant under $\operatorname{Autf}(\bar{M} / A)$, so does not fork over $A$, so by 2.11 is in $\operatorname{fact} \operatorname{bdd}(A)$-invariant.

We claim that in fact if $b$ and $c$ have the same compact strong type over $A$ (that is, the same type over $\operatorname{bdd}(A))$ then $\mu(\phi(x, b) \Delta \phi(x, c))=0$, which will show that $\mu$ is $\operatorname{bdd}(A)$-invariant. If not we have, without loss, $\mu(\phi(x, b) \wedge \neg \phi(x, c))>0$, so there is an ultrafilter $U$ containing $[\phi(x, b) \wedge \neg \phi(x, c)]$, and we obtain the global type $p_{U}$ which as pointed out above is $\operatorname{bdd}(A)$-invariant. As $\phi(x, b) \in p_{U}$, also $\phi(x, c) \in p_{U}$, a contradiction.

Note that, given small $A$ the collection of global $x$-types which do not fork over $A$ is closed in $S_{x}(\bar{M})$ and (assuming NIP and using 2.11) coincides with the space of global types invariant over bdd $(A)$. As in Section 8 , we call this space $S_{x}^{\text {bdd }(A)}(\bar{M})$. In the same vein as Proposition 4.5 we have.

Proposition 4.6. (Assume NIP.) Fix small A. Then there is a natural bijection between global Keisler measures which do not fork over A and regular Borel probability measures on $S_{x}^{\operatorname{bdd}(A)}(\bar{M})$.

Proof. We have already observed the bijection between global Keisler measures and regular Borel probability measures on the space of global types. Let $\mu_{x}$ be a global Keisler measure which does not fork over $A$, and let $\beta$ be the regular Borel measure on $S_{x}(\bar{M})$ corresponding to it. Then $\beta$ is concentrated on $S_{x}^{\operatorname{bdd}(A)}(\bar{M})$, hence its restriction to $S_{x}^{\operatorname{bdd}(A)}(\bar{M})$ determines $\beta$ and $\mu$. 
Proposition 4.7. (Assume NIP.) Let $p(x)$ be a complete type over A. Then the following are equivalent:

(i) $p$ does not fork over A (that is, $p$ has a global nonforking extension).

(ii) There is a global Keisler measure $\mu$ which extends $p$ and is A-invariant.

Proof. (ii) implies (i). Let $\mu$ be a global $A$-invariant Keisler measure which extends $p$ (in the obvious sense that any formula in $p$ gets measure 1). Suppose, for a contradiction, that $p(x)$ forks over $A$. So there are $\phi(x) \in p(x)$, and formulas $\psi_{1}(x), \ldots, \psi_{n}(x)$ (with parameters), each dividing over $A$, such that $\models \phi(x) \rightarrow \vee_{i} \psi_{i}(x)$. As $\mu(\phi(x))=1$, $\mu\left(\psi_{i}(x)\right)>0$ for some $i=1, \ldots, n$. But as $\mu$ is $A$-invariant, $\mu$ does not divide over $A$, contradiction.

(i) implies (ii). Let $p^{\prime}(x)$ be a global nonforking extension of $p$. By $2.11, p^{\prime}$ is $\operatorname{bdd}(A)$-invariant. By Proposition 2.6, $p^{\prime}$ is Borel definable over bdd( $A$ ). Let $G$ be the compact Lascar group over $A$, that is, $\operatorname{Aut}(\operatorname{bdd}(A) / A)$. Then $G$ is a compact group which thus has a (unique left and right invariant) Haar measure $h$. Let $S$ be the space of all complete types over bdd $(A)$. So $S$ is a compact space acted on continuously by $G$.

We now define the $A$-invariant global measure $\mu$ extending $p(x)$. Let $\phi(x, b)$ be a formula over $\bar{M}$. Let $q(y)=\operatorname{tp}(b / A)$, and let $Q \subset S$ be the space of complete types over bdd $(A)$ extending $q$ (a closed subset of $S$ ). The Borel definability of $p^{\prime}$ over bdd $(A)$ says that the set $X$ of $\operatorname{tp}\left(b^{\prime} / \operatorname{bdd}(A)\right)$ such that $\phi\left(x, b^{\prime}\right) \in p^{\prime}$ is a Borel subset of $S$. Hence $X \cap Q$ is also a Borel subset of $Q$. The compact space $Q$ is acted on continuously and transitively by $G$, hence has the form $G / H$ for some closed subgroup of $G$. Let $\pi$ be the canonical projection from $G$ onto $Q$. As $X \cap Q$ is Borel in $Q, \pi^{-1}(X \cap Q)$ is a Borel subset of $G$, hence $h\left(\pi^{-1}(X \cap Q)\right)$ is defined, and we define this to be $\mu(\phi(x, b))$. Note that if $\operatorname{tp}\left(b^{\prime} / A\right)=\operatorname{tp}(b / A)(=q)$ then by construction $\mu\left(\phi\left(x, b^{\prime}\right)\right)=\mu(\phi(x, b))$, so $\mu$ is $A$-invariant. Also if $\phi(x, b) \in p(x)$ (so $b \in A$ ), then $Q$ is a singleton $(\{\operatorname{tp}(b / A)\})$ and $X \cap Q=Q$, and hence $\mu(\phi(x, b))=1$. So $\mu$ extends $p$. We leave it to the reader to check finite additivity of $\mu$.

In the stable case it is not so hard to see, via the finite equivalence relation theorem for example, that the $A$-invariant measure $\mu$ extending $p$ in (ii) above is unique. Likewise if $p$ has a generically stable global nonforking extension. It would be interesting to find examples of uniqueness of $\mu$ when $p$ does not have a generically stable nonforking extension. We will come back to the issue of when $\mu$ is unique later in the paper.

We now return to the question of the Borel definability of invariant measures. We will make use of the Vapnik-Chervonenkis theorem [39] which we now describe.

Let $(X, \Omega, \mu)$ be a probability space, that is, a set $X$ equipped with a $\sigma$-algebra $\Omega$ of subsets (events) of $X$ and a countably additive probability measure $\mu$ with values in $[0,1]$. That is, $\mu(\emptyset)=0, \mu(X)=1, \mu(E)$ is defined for any $E \in \Omega$ and if $E_{i} \in \Omega$ for $i<\omega$ are pairwise disjoint and $E=\bigcup_{i} E_{i}$ then $\mu(E)=\sum_{i} \mu\left(E_{i}\right)$. For $k>0$, let $\mu^{k}$ be the product measure on $X^{k}$. Also, for $k>0, A \in \Omega$ and $\bar{p}=\left(p_{1}, \ldots, p_{k}\right) \in X^{k}$, let $\operatorname{fr}_{k}(A, \bar{p})$ be the proportion of $p_{i}$ 's which are in $A$, that is, $\left|\left\{i: p_{i} \in A\right\}\right| / n$. For any $A \in \Omega$, let $g_{A, k}: X^{k} \rightarrow[0,1]$ be defined by $g_{A, k}(\bar{p})=\left|\mathrm{fr}_{k}(A, \bar{p})-\mu(A)\right|$. Also let $h_{A, k}: X^{2 k} \rightarrow[0,1]$ be defined by $h_{A, k}(\bar{p}, \bar{q})=\left|\operatorname{fr}_{k}(A, \bar{p})-\operatorname{fr}_{k}(A, \bar{q})\right|$. Note that $g_{A, k}$ is $\mu^{k}$-measurable and $h_{A, k}$ is $\mu^{2 k}$-measurable. We will say (somewhat nonstandardly) that 
a family $\mathcal{C}$ of events has NIP if there is a natural number $d$ such that for no subset $F$ of $X$ of cardinality $d$ is it the case that $\{F \cap A: A \in \mathcal{C}\}$ is the full power set of $F$. For $\mathcal{C}$ with NIP the smallest such $d$ will be called the VC-dimension of $\mathcal{C}$.

With this notation, Theorem 2 of [39] gives:

VC Theorem. Suppose that the family $\mathcal{C}$ of events has NIP. Suppose also that for every $k>0, \sup _{A \in \mathcal{C}} g_{A, k}$ is $\mu^{k}$-measurable and $\sup _{A \in \mathcal{C}} h_{A, k}$ is $\mu^{2 k}$-measurable. Then there is a function $f(-,-)$ such that for any $\epsilon>0$ and $k<\omega, \mu^{k}\left(\left\{\bar{p}: \sup _{A \in \mathcal{C}} g_{A, k}(\bar{p})>\epsilon\right\}\right) \leq$ $f(k, \epsilon)$, and for any given $\epsilon, f(k, \epsilon) \rightarrow 0$ as $k \rightarrow \infty$. Moreover the function $f$ depends only on the VC-dimension of $\mathcal{C}$.

It is convenient to state a version or consequence of the VC Theorem in which the two measurability assumptions are dropped.

First let us note that any family $\mathcal{F}$ of measurable functions from $X$ to $[0,1]$, say, has an essential supremum, which is by definition measurable, and can be chosen as the outright supremum of some countable subfamily of $\mathcal{F}$. By an essential supremum of $\mathcal{F}$ we mean a measurable function $g: X \rightarrow[0,1]$ such that for each $f \in \mathcal{F}, g \geq f$ on a set of $\mu$-measure 1 , and whenever $g^{\prime}: X \rightarrow[0,1]$ has the same property, then $g \leq g^{\prime}$ on a set of measure 1. Such a $g$ can be found as follows. We construct measurable $g_{\alpha}$ for $\alpha$ a countable ordinal in the following way: Let $g_{0}$ be some member of $\mathcal{F}$. At limit stages, take suprema. Given $g_{\alpha}$, if it is already an essential supremum of $\mathcal{F}$, stop. Otherwise there is some $f \in \mathcal{F}$ such that $f>g_{\alpha}$ on a set of positive measure. Put $g_{\alpha+1}=\sup \left\{g_{\alpha}, f\right\}$. Note that $\int g_{\alpha}$ is strictly increasing with $\alpha$, hence the construction has to stop at some countable ordinal $\beta$. Then $g_{\beta}$ is measurable, is an essential supremum of $\mathcal{F}$, and is, by construction, the supremum of some countable subfamily of $\mathcal{F}$.

Note that any two essential suprema $g, g^{\prime}$ of $\mathcal{F}$ are equivalent in the sense that they are equal almost everwhere. We will just write ess $\sup \mathcal{F}$ for a representative of the equivalence class which we will assume to be the supremum of a countable subfamily of $\mathcal{F}$.

VC Theorem*. Suppose that the family $\mathcal{C}$ of events has NIP. Then for any $\epsilon>0, \mu^{k}(\{\bar{p}$ :

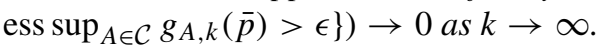

This *-version is immediately deduced from the first version: Let us fix $\epsilon$. We can find a countable subfamily $\mathcal{C}_{0}$ of $\mathcal{C}$ with the same VC-dimension, and such that for each $k$, $\operatorname{ess~sup}_{A \in \mathcal{C}} g_{A, k}=\sup _{A \in \mathcal{C}_{0}} g_{A, k}$. Note that $\sup _{A \in \mathcal{C}_{0}} h_{A, k}$ is measurable. Hence we can apply the VC Theorem with $\mathcal{C}_{0}$ in place of $\mathcal{C}$ and we obtain the *-version.

We will apply the above theorems to the following situation. Let $M$ be a model of a NIP theory, $\phi(x, y)$ an $L$-formula, and $\mu_{x}$ a Keisler measure over $M$. Take $X$ to be the Stone space $S_{x}(M), \Omega$ the $\sigma$-algebra of its Borel subsets, and identify $\mu_{x}$ with the measure induced on $X$ as described at the beginning of this section. Let $\mathcal{C}$ be the family of clopen subsets of $X$ given by the formulas $\phi(x, c)$ as $c$ varies over $M$. It is easy to see that as $T$ has $N I P$, the family $\mathcal{C}$ has $N I P$.

Lemma 4.8. (Assume NIP.) Let $M$ be a model and $\mu_{x}$ a Keisler measure over $M$.

(i) Let $\phi(x, y) \in L$, and let $\epsilon>0$. Then there is $k$ and a Borel subset $B$ of $S_{x}(M)^{k}$ of positive $\mu^{k}$ measure such that for all $\left(p_{1}, \ldots, p_{k}\right) \in B$ and any $c \in M, \mu(\phi(x, c))$ is within $\epsilon$ of the proportion of the $p_{i}$ which contain $\phi(x, c)$. 
(ii) If moreover $M$ is saturated (for example equals $\bar{M}$ ) and $A \subseteq M$ is small such that $\mu$ does not fork over $A$, then in (i) we can choose $B$ such that for any $\left(p_{1}, \ldots, p_{k}\right) \in B$, no $p_{i}$ forks over $A$.

Proof. (i) We will identify $\phi(x, c)$ with the clopen subset of $X$ it defines, and as above write $\operatorname{fr}_{k}(\phi(x, c), \bar{p})$ for the proportion of $p_{i}$ containing $\phi(x, c)$. We fix our $\epsilon>0$. By VC Theorem*, choose $k$ and $\delta>0$ such that $\mu^{k}\left(\left\{\bar{p}: \operatorname{ess}^{*} \sup _{A \in \mathcal{C}} g_{A, k}(\bar{p})<\epsilon\right\}\right)>\delta$. It follows that for each finite set $C=\left\{c_{1}, \ldots, c_{m}\right\}$ of parameters from $M$, the clopen set $B_{C}=\left\{\left(p_{1}, \ldots, p_{k}\right) \in S(M)^{k}: \operatorname{fr}_{k}\left(\phi\left(x, c_{j}\right), \bar{p}\right)-\mu\left(\phi\left(x, c_{j}\right)\right)<\epsilon\right.$ for $\left.j=1, \ldots, m\right\}$ has $\mu^{k}$-measure $>\delta$. Note that $B_{C^{\prime}} \subseteq B_{C}$ when $C \subseteq C^{\prime}$. Let $B$ be the intersection of all $B_{C}$. Clearly it works.

(ii) This is proved exactly as (i) but working in the closed set $S_{x}^{\text {bdd( }(A)}(M)$ of $S_{x}(M)$ consisting of types over $M$ which do not fork over $A$, and bearing in mind 4.6.

Corollary 4.9. (Assume NIP.) Suppose that $\mu$ is a global Keisler measure which does not fork over A. Then $\mu$ is Borel definable over bdd $(A)$.

Proof. Fix $\phi(x, y) \in L$ and $\epsilon>0$. Let $p_{1}, \ldots, p_{n} \in B$ where $B$ is given by Lemma 4.8(ii) (for $M=\bar{M}$ ). By Proposition 2.6, each $p_{i}$ is strongly Borel definable over $\operatorname{bdd}(A)$. Then for each $i$ there is some finite Boolean combination $Y_{i}$ of type-definable over $\operatorname{bdd}(A)$-sets such that for all $c, \phi(x, c) \in p_{i}$ iff $c \in Y_{i}$. It follows that if $c, c^{\prime}$ lie in exactly the same $Y_{i}$ for $i=1, \ldots, n$, then $\mu(\phi(x, c))$ and $\mu\left(\phi\left(x, c^{\prime}\right)\right)$ differ by $<2 \epsilon$. This suffices to prove Borel definability of $\mu$.

A thorough account of the model-theoretic results related to VC-type theorems will appear in a forthcoming paper.

\section{Generics and forking in definable groups}

In [16] the $f s g$ (finitely satisfiable generics) property for definable groups was introduced. Strengthenings of the $f s g$ will be discussed in Section 6. In this section we discuss weakenings of the $f s g$, relate the $f_{s} g$ to forking, and in general try to obtain equivariant versions of results from Section 2 .

Let $G$ be a $\emptyset$-definable group in $\bar{M} . S_{G}(A)$ denotes the set of complete types over $A$ extending " $x \in G$ ". Recall from [16] and [38] that if $T$ has NIP then $G^{00}$ (the smallest type-definable bounded index subgroup of $G$ ) exists. Our notion of "generic" will be from [16]. However in [14] "generic" is used differently (there it is a translation invariant definable type). Also we distinguish it (as in [23]) from " $f$-generic":

Definition 5.1. (i) A definable subset $X$ of $G$ is said to be left generic if finitely many left translates of $X$ by elements of $G$ cover $G$.

(ii) $p(x) \in S_{G}(A)$ is left generic if every formula in $p$ is left generic.

(iii) A definable subset $X$ of $G$ is said to be left $f$-generic if for each $g \in G, g X$ does not fork over $\emptyset$.

(iv) $p(x) \in S_{G}(A)$ is left $f$-generic if every formula in $p$ is left $f$-generic. 
(v) $G$ has the $f_{s} g$ if there is a global type $p(x) \in S_{G}(\bar{M})$ such that every left translate $g p$ of $p$ is almost finitely satisfiable over $\emptyset$, that is, for every model $M_{0}$, every left translate of $p$ is finitely satisfiable in $M_{0}$.

Note that if $G$ is the additive group in $R C F$ (or just the underlying group in DOAG) then there are no complete generic types, but there are two $f$-generic types, at $+\infty$ and $-\infty$. (Both these types are definable over $\emptyset$ and invariant under the group operation).

In [16] we pointed out:

Fact 5.2. G has the $f_{s} g$ if and only if the following three conditions hold.

(a) The class of left generic definable subsets of $G$ coincides with the class of right generic sets (we call them simply generic).

(b) The class of nongeneric definable subsets of $G$ is a (proper) ideal.

(c) If $X$ is a generic definable subset of $G$, then $X$ meets every model $M_{0}$.

The $f s g$ is a rather strong property for a definable group. It is a kind of abstract "definable compactness". The following was observed in [16, Theorem 8.1 and Remark 3 of Section 8].

Remark 5.3. In $o$-minimal expansions of real closed fields, the definable groups with $f s g$ are precisely the definably compact groups.

Definable groups in algebraically closed valued fields have been studied by the first author ([14]). From the analysis there, one can, with additional work, obtain a characterization of the definable groups $G$ with $f s g$, at least when $G$ is abelian: they are precisely the (abelian) groups $G$ such that there is a definable homomorphism $h: G \rightarrow A$ where $A$ is internal to the value group and is definably compact, and $\operatorname{Ker}(h)$ is stably dominated. The $p$-adically closed field case is considered in [24], where among other things it is pointed out that definably compact groups defined over the standard model have $f s g$.

Corollary 5.4. If $G$ has the fsg, then there exists a (simultaneously left and right) global generic type $p \in S_{G}(\bar{M})$ and moreover any such global generic type is also (left and right) $f$-generic.

Problem 5.5. Suppose $G$ has the $f_{s} g$ and assume NIP if one wants. Is it the case that every left $f$-generic type is generic?

In the case of definably compact groups in o-minimal expansions of real closed fields, Problem 5.5 has a positive answer. This follows from the results in [7] and [26] which give that: if $X$ is definable, closed and bounded then $X$ does not fork over $M_{0}$ if and only if $X$ meets $M_{0}$. So assume that $X$ is left $f$-generic and we want to prove that $X$ is generic. We may assume $X$ is closed. Let $M_{0}$ be any small model. So for any $g \in G, g X$ meets $M_{0}$. By compactness, $X$ is right generic in $G$ (so generic in $G$ ).

We now work without the $f s g$ assumption, improving a result from [16]. 
Proposition 5.6. (Assume NIP.) Suppose that $G$ has a global left $f$-generic type $p$. Then

(i) $\operatorname{Stab}_{l}(p)=G^{00}=G^{000}$,

(ii) $G$ is definably amenable, that is, $G$ has a left invariant global Keisler measure.

Proof. Recall that $\operatorname{Stab}_{l}(p)$, the left stabilizer of $p$, is by definition the set of $g \in G$ such that $g p=p$. It is a subgroup of $G$ but on the face of it has no definability properties.

(i) Let us fix a small model $M_{0}$ for convenience.

Claim I. If $\operatorname{tp}\left(g_{1} / M_{0}\right)=\operatorname{tp}\left(g_{2} / M_{0}\right)$ then $g_{1}^{-1} g_{2} \in \operatorname{Stab}_{l}(p)$.

Proof of Claim I. By $f$-genericity of $p$ and the NIP assumption, both $p$ and $g_{1} p$ are Aut $_{M_{0}}(\bar{M})$-invariant. Let $f \in \operatorname{Aut}_{M_{0}}(\bar{M})$ be such that $f\left(g_{1}\right)=g_{2}$. So $g_{1} p=f\left(g_{1} p\right)=$ $f\left(g_{1}\right) f(p)=g_{2} p$. Hence $g_{1}^{-1} g_{2} \in \operatorname{Stab}_{l}(p)$.

Claim II. Suppose $h \in \operatorname{Stab}_{l}(p)$. Then $h=g_{1}^{-1} g_{2}$ for some $g_{1}, g_{2}$ with the same type over $M_{0}$.

Proof of Claim II. By definition $h a=b$ for some realizations of $p$ (in a model containing $\bar{M}$ ). So we can find realizations $a, b$ of $p \mid M_{0}$ in $\bar{M}$ such that $h a=b$. So $h=b a^{-1}$. Put $g_{1}=b^{-1}$ and $g_{2}=a^{-1}$ and note they have the same type over $M_{0}$.

From Claims I and II we deduce that $\operatorname{Stab}_{l}(p)$ is type-definable (over $M_{0}$ ) as the set of $g_{1}^{-1} g_{2}$ for $g_{1}, g_{2} \in \bar{G}$ having the same type over $M_{0}$. On the other hand by Claim I, the index of $\operatorname{Stab}_{l}(p)$ in $G$ is bounded by the number of types over $M_{0}$. It follows that $\operatorname{Stab}_{l}(p)$ has to be $G^{00}$, because $p$ determines a coset of $G^{00}$. The same reasoning yields $\operatorname{Stab}_{l}(p)=G^{000}$.

(ii) This follows by the same proof as that of Proposition 6.2 of [16], using part (i) together with Proposition 2.6: We can reduce to the case where $T$ is countable. Given $X$ a definable subset of $G$, let $M_{0}$ be a countable model over which $X$ is defined. Note that $p$ does not fork over $M_{0}$. Proposition 2.6 shows that not only is $p$ Borel definable over $M_{0}$, but also (using countability of the language and of $M_{0}$ ) any " $\phi(x, y)$-definition of $p$ " is a countable union of closed sets over $M_{0}$. In particular $Y=\{g \in G: X \in g p\}$ is a countable union of closed sets over $M_{0}$. By part (i) whether or not $g \in Y$ depends only on the coset $g / G^{00}$. Let $\pi: G \rightarrow G / G^{00}$ be the canonical surjection. Then $\pi(Y)$ is a Borel subset of $G / G^{00}$ and we define $\mu(X)$ to be $\mathbf{h}(\pi(Y))$ where $\mathbf{h}$ is the unique normalized Haar measure on the compact group $G / G^{00}$. Then $\mu$ is clearly additive and is also left invariant: Given $h \in G, \mu(h X)=\mathbf{h}\left(\left\{g / G^{00}: h X \in g p\right\}\right)$. But $h X \in g p$ iff $g=h g^{\prime}$ for some $g^{\prime}$ such that $X \in g^{\prime} p$. Hence $\left\{g / G^{00}: h X \in g p\right\}=h / G^{00} \cdot\left\{g^{\prime} / G^{00}: X \in g p\right\}$. So by left invariance of $\mathbf{h}, \mu(h X)=\mathbf{h}\left(\left\{g^{\prime} / G^{00}: X \in g p\right\}\right)=\mu(X)$.

Remark 5.7. Proposition 5.6 also holds when $G$ is type-definable (with appropriate definitions). But for $G$ definable (over $\emptyset$ ), we could also deduce 5.6 from 4.7 and 2.10(i), together with Construction $\mathrm{C} 1$ from the introduction. (Assume NIP.) Let $T^{\prime}$ be the theory $T$ together with an additional sort $S$ and a $\emptyset$-definable regular action of $G$ on $S$. Let $x$ be a variable of sort $S$. Then the following are equivalent: (i) In $T$ there is a global left $f$-generic type, (ii) in $T^{\prime}, x=x$ does not fork over $\emptyset$. 
We now aim towards a kind of converse of 5.6. First:

Lemma 5.8. (Assume NIP.) Let $G$ be a definable (or even type-definable) group, and let $\mu$ be a global left invariant Keisler measure on $G$. Let $M_{0}$ be any small model over which $G$ is defined. Then there is a global left invariant Keisler measure $\mu^{\prime}$ on $G$ such that $\mu^{\prime}\left|M_{0}=\mu\right| M_{0}$ and $\mu^{\prime}$ is definable (hence $\operatorname{Aut}(\bar{M} / M)$-invariant for some small $M$ ).

Proof. In this and two subsequent lemmas $(7.6,8.10)$ we will make mild use of the compactness of the space of Keisler measures over a set $A$, which we now explain. Let $A$ be a set of parameters, and consider the set $\mathcal{M}_{x}(A)$ of Keisler measures $\mu_{x}$ over $A$. We obtain a topology on $\mathcal{M}_{x}(A)$ by taking a basic closed set to be $\left\{\mu_{x}: \mu(\phi(x)) \in I\right\}$ for $\phi(x)$ a formula over $A$ and $I$ a closed subset of $[0,1]$. With this topology, $\mathcal{M}_{x}(A)$ is clearly a closed subset of the product space $[0,1]^{L_{x}(A)}$ (where $L_{x}(A)$ denotes the collection of $A$ definable sets of the relevant sort), hence, using Tikhonov's theorem, $\mathcal{M}_{x}(A)$ is compact. Alternatively, compactness of $\mathcal{M}_{x}(A)$ is given by Lemma 1.5 of [20], and also follows from Construction (*) in Section 2 of [16]. If $G$ is a definable group, $M$ is a model over which $G$ is defined, and the variable $x$ ranges over $G$, then the set of left $G(M)$-invariant Keisler measures $\mu_{x}$ over $M$ is a closed subset of $\mathcal{M}_{x}(M)$, so also compact.

We now turn to the proof of the lemma, which follows Keisler's construction in [20] of "smooth measures" assuming NIP.

Step I. We construct $M$ extending $M_{0}$ and extension $\mu_{1}$ of $\mu \mid M_{0}$ to a left $G(M)$-invariant measure over $M$ such that $\mu_{1}$ has a unique extension to a global left invariant measure $\mu^{\prime}$.

Suppose that already $\mu$ and $M_{0}$ do not work. So $\mu \mid M_{0}$ has two distinct extensions $\lambda, \beta$ to left invariant global measures. So there is a definable subset $X$ of $G$ and some positive real number $r$ such that $|\lambda(X)-\beta(X)|>r$. Let $\mu^{\prime}$ be the average of $\lambda$ and $\beta$. Note that $\mu^{\prime}$ has the property

(*) for any $M_{0}$-definable subset $Y$ of $G, \mu^{\prime}(X \triangle Y)>r / 4$.

(Otherwise $\mu^{\prime}(X \triangle Y) \leq r / 4$ for some $M_{0}$-definable $Y$. Hence $\lambda(X \triangle Y)+\beta(X \triangle Y)$ $\leq r / 2$. But $\lambda(Y)=\beta(Y)=\mu(Y)$, so we would deduce that $|\lambda(X)-\beta(X)| \leq r$, a contradiction.)

Let $M_{1}$ be a small model containing $M_{0}$ such that $X$ is over $M_{1}$, and let $\mu_{1}$ be the restriction of $\mu^{\prime}$ to $M_{1}$. Continue with $\mu_{1}$ in place of $\mu \mid M_{0}$. Let us first point out that this construction can be continued at limit ordinals. Suppose for example that we have constructed $\mu_{i}$ over $M_{i}$, for $i<\omega$, such that for $i<j, M_{i} \subset M_{j}, \mu_{i} \subset \mu_{j}$, and each $\mu_{i}$ is $G\left(M_{i}\right)$-invariant, and extends to a global $G$-invariant Keisler measure. We need to know that $\bigcup_{i} \mu_{i}$ can also be extended to a global $G$-invariant Keisler measure, and this follows from compactness of the space of $G$-invariant global Keisler measures. Alternatively (as pointed out by the referee) note that $\bigcup_{i} \mu_{i}$ is a $G(M)$-invariant Keisler measure over $M=\bigcup_{i} M_{i}$, and we can directly obtain a global $G$-invariant Keisler measure extending $\bigcup_{i} \mu_{i}$ from Construction $(*)$ of [16].

Now we claim that at some point we arrive at $\mu_{\alpha}$ over a model $M_{\alpha}$ which is left $G\left(M_{\alpha}\right)$-invariant and has a unique global left invariant extension. Otherwise by $(*)$ we find a formula $\phi(x, y)$, a positive real number $\epsilon$ and $\left(c_{i}: i<\omega\right)$, and a global measure 
$\mu^{\prime \prime}$ such that $\mu^{\prime \prime}\left(\phi\left(x, c_{i}\right) \Delta \phi\left(x, c_{j}\right)\right)>\epsilon$ for all $i \neq j$. This (together with NIP) is a contradiction. (See 3.14 of [20] or 3.3 of [16].) So Step I is accomplished.

Step II. $\mu^{\prime}$ from Step I is definable (over $M$ ). This is proved (via Beth's theorem) exactly as in the proof of Lemma 2.6 (definability of minimal measures) in [16].

The following consequence of Lemma 5.8 was also noticed by Krzysztof Krupiński.

Proposition 5.9. (Assume NIP.) Suppose that the definable group $G$ is definably amenable (has a global left invariant Keisler measure). Then, after possibly adding constants for some small model over which $G$ is defined, there is a global left $f$-generic type of $G$.

Proof. By Lemma 5.8, there is a left invariant global Keisler measure $\mu$ on $G$ which is moreover definable, over some small model $M$, hence does not fork over $M$. Let $p$ be a global type of $G$ such that $\mu(X)>0$ for every $X \in p$. Then for every $X \in p$ and $g \in G$, $\mu(g X)=\mu(X)>0$, hence $g X$ does not fork over $M$. So $p$ is a left $f$-generic of $G$ after adding names for the model $M$.

So we obtain the following:

Corollary 5.10. (Assume NIP.) $G$ is definably amenable if and only if $G$ has a global left $f$-generic type after possibly adding constants. Moreover under these circumstances $G^{000}$ exists and coincides with $G^{00}$.

The corollary goes through for type-definable $G$ too. Shelah [38] has recently proved that assuming $N I P$, for any abelian type-definable group $G, G^{000}$ exists. As abelian groups are amenable, the corollary above improves Shelah's theorem. On the other hand Gismatullin [9] has recently been able to drop the abelian hypothesis from Shelah's theorem.

The following clarifies the question of the existence of left $f$-generic types and addition of constants. In particular the proof of (ii) gives another example where forking does not equal dividing.

Proposition 5.11. (Assume NIP.)

(i) Suppose $G$ is a definable group and has a global left $f$-generic type after naming elements of some model. Then $G$ has a global left $f$-generic type after naming elements of any model over which $G$ is definable.

(ii) There is a Ø-definable group $G$ in a theory $T$ with NIP, such that $G$ is definably amenable, but there is no global $f$-generic type of $G$.

Proof. (i) We assume that $G$ is $\emptyset$-definable in $T$. We will use Remark 5.7. That is, we work in the theory $T^{\prime}$ which is $T$ together with a new sort $S$ and a regular right action of $G$ on $S$. Moreover, $\bar{M}$ is our monster model of $T$ and $\bar{M}^{\prime}$ denotes the corresponding (monster) model of $T^{\prime}$, that is, $\bar{M}$ together with the new sort $S$. Let $x$ be a variable of sort $S$. By 5.7 it is enough to prove:

Claim. Suppose $x=x$ forks over the small elementary substructure $M_{0}$ of $\bar{M}$. Then $x=x$ forks over any small elementary substructure of $\bar{M}$ containing $M_{0}$.

Proof of Claim. Assume for simplicity that $x=x$ implies $\phi(x, a) \vee \psi(x, b)$ where each of $\phi(x, a), \psi(x, b)$ divides over $M_{0}$ (in $\left.\bar{M}^{\prime}\right)$. Let $\left(a_{i}: i<\omega\right)$ and $\left(b_{i}: i<\omega\right)$ 
be infinite $M_{0}$-indiscernible sequences in $\operatorname{tp}\left(a / M_{0}\right)$ and $\operatorname{tp}\left(b / M_{0}\right)$ respectively, such that $\left\{\phi\left(x, a_{i}\right): i<\omega\right\}$ and $\left\{\psi\left(x, b_{i}\right): i<\omega\right\}$ are each inconsistent. Let $M_{1}$ be a small elementary substructure of $\bar{M}$ containing $M_{0}$. By an automorphism argument we may assume that $\operatorname{tp}\left(M_{1} / M_{0} \cup\left\{a_{i}: i<\omega\right\} \cup\left\{b_{i}: i<\omega\right\}\right)$ is finitely satisfiable in $M_{0}$. But then clearly each of the sequences $\left(a_{i}: i<\omega\right)$ and $\left(b_{i}: i<\omega\right)$ is also $M_{1}$-indiscernible. So $x=x$ forks over $M_{1}$.

(ii) Let $K$ be a (saturated) real closed field. Let $G$ be $K \times K$, equipped with its addition, and with all relations which are $\emptyset$-definable in $(K,+, \cdot)$ and invariant under the action of $\operatorname{SL}(2, K)$. Of course the theory of $G$ with this structure has NIP, and $G$ is definably amenable as it is abelian. Suppose for a contradiction that $G$ had a global $f$-generic type $p(x)$. In particular $p(x)$ does not fork over $\emptyset$, so is invariant under automorphisms fixing bdd $(\emptyset)$ by Section 2 . As $\operatorname{SL}(2, K)$ acts on $G$ by automorphisms and $\operatorname{SL}(2, K)^{00}=\operatorname{SL}(2, K)$, it follows that $p$ is $\operatorname{SL}(2, K)$-invariant. But then $p$ induces a $\operatorname{PSL}(2, K)$-invariant global type of the projective line over $K$, which contradicts Remark 5.2(iv) of [16] and its proof.

One might think that any global left generic type of any definable group is automatically left $f$-generic (NIP or no NIP). But we need some assumptions to prove it.

Proposition 5.12. (Assume NIP.). Let $G$ be a definable group which is definably amenable. Then any global left generic type of $G$ is also left $f$-generic.

Proof. It is clearly sufficient to prove that any left generic definable set $X$ does not divide over $\emptyset$. And for this it is enough to find a small model $M_{0}$ such that any left generic definable set $X$ does not divide over $M_{0}$. (Given left generic $X_{a}$ defined over $a$, and an indiscernible sequence $\left(a_{i}: i<\omega\right)$ in $\operatorname{tp}(a / \emptyset)$, we can find a sequence $\left(a_{i}^{\prime}: i<\omega\right)$, indiscernible over $M_{0}$ and with the same type over $\emptyset$ as $\left(a_{i}: i<\omega\right)$.)

The assumption that there is a global left generic type implies easily that the class $\mathcal{I}$ of non-left generic definable sets is a proper ideal of the Boolean algebra of all definable subsets of $G$. By Proposition 6.3(i) of [16], there is a small model $M_{0}$ such that for every left generic definable set $X$ there is some $M_{0}$-definable subset $Y$ of $G$ such that the symmetric difference $X \triangle Y$ is in $\mathcal{I}$, so is non-left generic.

Now suppose that $X_{a}$ is a left generic definable subset of $G$ (defined over $a$ ). We want to show that $X_{a}$ does not divide over $M_{0}$. Let $Y$ be definable over $M_{0}$ such that the symmetric difference of $X_{a}$ and $Y$ is nongeneric. Replacing $X_{a}$ by $X_{a} \cap Y$ we may assume that $X_{a} \subseteq Y$. Let $\left(a_{i}: i<\omega\right)$ be an $M_{0}$-indiscernible sequence with $a_{0}=a$. Suppose by way of contradiction that $\bigcap_{i=1}^{n} X_{a_{i}}$ is inconsistent for some $n$. Then (as $Y$ is defined over $M_{0}$ ), the union of the $Y \backslash X_{a_{i}}$ for $i=1, \ldots, n$ covers $Y$. But $Y$ is left generic, and each $Y \backslash X_{a_{i}}$ is non-left generic, contradicting $\mathcal{I}$ being an ideal. This proves the proposition.

\section{Generically stable groups}

In this section we introduce various strengthenings of the $f s g$ leading to the notion of a generically stable group (analogous to a generically stable type). Assuming NIP these strengthenings will be equivalent. 
We first give a natural definition of the $f s g$ for type-definable groups.

Definition 6.1. Let $G$ be a type-definable group. We say that $G$ has $f s g$ if there is some small model $M_{0}$ and global type $p(x)$ of $G$ such that $g p$ is finitely satisfiable in $G\left(M_{0}\right)$ for any $g \in G$.

The basic results on definable groups with $f_{s} g$ go through easily for type-definable groups $G$. Namely we define a relatively definable subset $X$ of $G$ to be left generic if finitely many left translates of $X$ by elements of $G$ cover $G$.

Lemma 6.2. Suppose $G$ is type-definable with $f_{s} g$. Then:

(i) The class of relatively definable left generic subsets of $G$ coincides with the class of relatively definable right generic subsets of $G$ and forms a proper ideal of the class of relatively definable subsets of $G$.

(ii) There is a global generic type of $G$, that is, a global type $p$ extending $x \in G$ such that any relatively definable subset of $G$ which is in $p$ is generic.

(iii) There is a small model $M_{0}$ such that any global generic type of $G$ is finitely satisfiable in $G\left(M_{0}\right)$, and such that moreover if $X$ is a relatively definable generic subset of $G$ then finitely many (left or right) translates of $X$ by elements of $G\left(M_{0}\right)$ cover $G$.

(iv) $G^{00}$ exists and is both the left and right stabilizer of any global generic type of $G$.

Definition 6.3. Let $G$ be a definable group.

(i) We say that $G$ has $f_{s} g^{+}$if $G$ has $f s g$ and some global generic type is definable.

(ii) We say that $G$ has $f_{s} g^{++}$if $G^{00}$ exists and has $f s g$ as a type-definable group in its own right.

(iii) We say that $G$ is generically stable if $G$ has $f s g$ and some global generic type is generically stable.

Note that (ii) seems to be a rather minor variation on $G$ having $f s g$ but in fact it is a substantial strengthening. Note in particular that if $G=G^{00}$ and $G$ has the $f s g$ then it has $f_{s} g^{++}$. Definably compact groups in $o$-minimal structures satisfy none of the above properties, although they have $f_{s} g$. We will see soon that (iii) implies (ii) implies (i) and that they are all equivalent assuming NIP.

Lemma 6.4. Suppose G has $f_{s}{ }^{++}$. Then:

(i) G has fsg.

(ii) Any global generic type of $G^{00}$ is a global generic type of $G$.

(iii) There is a unique global generic type of $G$ which implies " $x \in G^{00}$ ”, say $p_{0}$.

(iv) $p_{0}$ is definable and is also the unique global generic type of $G^{00}$.

Proof. (i) Let $M_{0}$ be a small model witnessing that $G^{00}$ has $f_{s} g$. We may assume that $M_{0}$ contains representatives of each coset of $G^{00}$ in $G$ (as there are boundedly many). Let $p$ be a global generic type of $G^{00}$ and $g \in G$. Then $g_{1}^{-1} g \in G^{00}$ for some $g_{1} \in G\left(M_{0}\right)$. By assumption $g_{1}^{-1} g p$ is finitely satisfiable in $G\left(M_{0}\right)$ (in fact in $G^{00}\left(M_{0}\right)$ ). Hence so is $g p$. This shows that $G$ has $f s g$.

(ii) We have shown in (i) that if $p$ is a global generic of $G^{00}$ then also $g p$ is finitely satisfiable in $G\left(M_{0}\right)$ for any $g \in G$, which implies that $p$ is a global generic type of $G$. 
(Alternatively if $X$ is a definable subset of $G$ and $X \cap G^{00}$ is generic in $G^{00}$ then finitely many translates of $X$ cover $G^{00}$, so finitely many translates of this set of finitely many translates cover $G$.)

(iii) Suppose for a contradiction that $p$ and $q$ are distinct global generic types of $G$ each of which implies that " $x \in G^{00 "}$ ". Let $X$ be a definable set in $p$ such that $Y=G \backslash X$ is in $q$. Let $X_{0}=X \cap G^{00}$ and $Y_{0}=Y \cap G^{00}$. So $X_{0}$ and $Y_{0}$ are relatively definable subsets of $G^{00}$ which partition $G^{00}$. By Lemma 6.2 we may assume that $X_{0}$ is generic in $G^{00}$. So finitely many translates of $X_{0}$ by elements of $G^{00}$ cover $G^{00}$. In particular (as $q(x)$ implies $\left.x \in G^{00}\right)$, there is $g \in G^{00}$ such that $g X \in q$. But by Corollary 4.3 of [16] the symmetric difference of $X$ and $g X$ is nongeneric (in $G$ ), so as $q$ is a generic type of $G$, we see that $X$ is also in $q$, a contradiction.

(iv) Let $p_{0}$ be the unique type from (iii). By part (ii), $p_{0}$ is also the unique global generic type of $G^{00}$. Let $G^{00}$ be the intersection of the directed family $\left(Y_{i}\right)_{i \in I}$ of definable subsets of $G$. For a given $L$-formula $\phi(x, y)$ and $c \in \bar{M}, \phi(x, c) \in p_{0}$ iff for some $i \in I$ finitely many translates of (the set defined by) $\phi(x, c)$ by elements of $G^{00}\left(M_{0}\right)$ cover $Y_{i}$. The same is true for $\neg \phi(x, y)$. By compactness the set of $c$ such that $\phi(x, c) \in p_{0}$ is definable. So $p_{0}$ is definable.

Proposition 6.5. Let $G$ be a definable group. Then:

(i) If $G$ has $f g^{++}$then $G$ has $f s g^{+}$.

(ii) If $G$ is generically stable then $G$ has $f_{s} g^{++}$.

(iii) Assume NIP. If $G$ has $f_{s g}{ }^{+}$then $G$ is generically stable. (Hence assuming NIP the three properties $\mathrm{fsg}^{+}, \mathrm{fsg}^{++}$and generic stability are equivalent.)

Proof. (i) follows from the previous lemma.

(ii) By translating we obtain a generically stable global generic type $p(x)$ of $G$ such

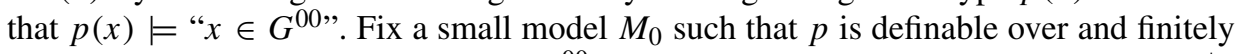
satisfiable in $M_{0}$ (and of course $G$ and $G^{00}$ are defined over $M_{0}$ ). Let $M$ be a small $\left|M_{0}\right|^{+}$saturated extension of $M_{0}$. Let $\left(a_{i}: i<\omega\right)$ be a Morley sequence in $p$ over $M_{0}$ inside $M$. Then $a_{i} \in G^{00}(M)$ for all $i$. Suppose $\phi(x, c) \in p$. The generic stability of $p$ implies that $\phi(x, c)$ is satisfied by some $a_{i}$, so $\phi(x, c)$ is satisfied in $G^{00}(M)$. If $g \in G^{00}$ then $g p=p$ hence any left translate of $p$ by an element of $G^{00}$ is finitely satisfiable in $G^{00}(M)$, so $G^{00}$ has $f s g$ and $G$ has $f s g^{++}$.

(iii) is immediate because assuming NIP any global type which is both definable over and finitely satisfiable in some small model, is generically stable.

We now develop some consequences of the "weakest" of the new properties, $f_{s g}{ }^{+}$.

Proposition 6.6. Suppose that the definable group $G$ has $f_{s g}{ }^{+}$. Then:

(i) $G^{00}=G^{0}$.

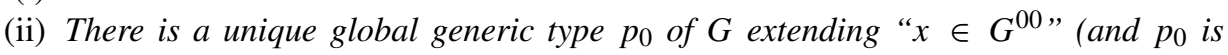
definable).

(iii) Every global generic type of $G$ is definable and the set of global generics is in oneone correspondence with $G / G^{00}$. 
(iv) For any $L$-formula $\phi(x, y)$ there is $N<\omega$ such for any $c, \phi(x, c)$ is generic in $G$ if and only if at most $N$ left (right) translates of $\phi(x, c)$ cover $G$.

Proof. (i) Fix a definable global generic type $p$. By 4.3 from [16] we know that $\operatorname{Stab}(p)=$ $G^{00}$. For a fixed formula $\phi(x, y)$ let (just for now) $p \mid \phi$ be the set of formulas of the form $\phi\left(g^{-1} x, c\right)$ which are in $p$ for $g \in G$ and $c \in \bar{M}$. Then $\operatorname{Stab}_{\phi}(p)$ denotes $\{g \in G$ : $g p|\phi=p| \phi\}$ and is a definable subgroup of $G$. As $\operatorname{Stab}(p)=\bigcap_{\phi \in L} \operatorname{Stab}_{\phi}(p)$ it follows that each $\operatorname{Stab}_{\phi}(p)$ has finite index and so $G^{00}$ is the intersection of a family of definable subgroups of finite index, so equals $G^{0}$.

(ii) By translating the given definable generic $p$ we can find a definable global generic type which extends " $x \in G^{00 "}$ " and we call it $p_{0}$. Note that if $q(x)$ is a global generic

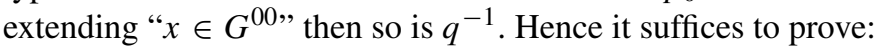

Claim. If $q$ is any global generic type extending " $x \in G^{00 "}$ then $q^{-1}=p_{0}$.

Proof of Claim. Let $M_{0}$ be any model. So $q$ is finitely satisfiable in $M_{0}$ and $p_{0}$ is definable over $M_{0}$. Let $a$ realize $p_{0} \mid M_{0}$ and $b$ realize $q \mid\left(M_{0}, a\right)$. As $a \in G^{00}$ and $G^{00}=\operatorname{Stab}(q)$ it follows that $c=a b$ also realizes $q \mid\left(M_{0}, a\right)$. By Lemma 3.4, $a$ realizes $p_{0} \mid\left(M_{0}, c\right)$. Again as $c^{-1} \in G^{00}$ we see that $c^{-1} a=b^{-1}$ also realizes $p_{0} \mid\left(M_{0}, c\right)$. In particular $\operatorname{tp}\left(b^{-1} / M_{0}\right)=p_{0} \mid M_{0}$. As $M_{0}$ was arbitrary it follows that $q^{-1}=p_{0}$. The claim is proved as well as part (ii).

(iii) Now any global generic type of $G$ is a translate of a global generic which implies " $x \in G^{00 "}$. By (ii) every global generic type of $G$ is a translate of the definable type $p_{0}$, hence is also definable.

(iv) If $\phi(x, y) \in L$ and $c \in \bar{M}$, then $\phi(x, c)$ is generic in $G$ if for some $g \in G$, $\phi\left(g^{-1} x, c\right) \in p_{0}$. So by definability of $p_{0}$ the set of $c$ such that $\phi(x, c)$ is generic is definable, which is enough.

Let us remark that there are groups with $G^{00}=G^{0}$, with $f_{s} g$ but without $f_{s} g^{+}$(even in a NIP environment). The easiest example is simply the additive group $R^{+}$of the valuation ring $R$ in a saturated $p$-adically closed field. Here $\left(R^{+}\right)^{00}$ is the intersection of the finite index definable subgroups $v(x) \geq n$ for $n \in \mathbb{Z}^{+}$, but there are many generic types extending $\left(R^{+}\right)^{00}$.

Remark 6.7. Suppose the definable group $G$ is generically stable. Let $X$ be a definable subset of $G$. Then $X$ is generic if and only if every left (right) translate of $X$ does not divide (or even fork) over $\emptyset$.

Proof. We know left to right by just the $f$ sg. Now suppose that $X$ is nongeneric, and defined over some model $M_{0}$. Let $p$ be some generically stable global generic type of $G$, and let $\left(a_{i}: i<\omega\right)$ be a Morley sequence of $p$ over $M_{0}$. Then for any $g \in G$, as $g X \notin p$, we see that $a_{i} \notin g X$ for some $i<\omega$. It follows that $\left\{a_{i}^{-1} X: i<\omega\right\}$ is inconsistent. As $\left(a_{i}^{-1}: i<\omega\right)$ is indiscernible over $M_{0}$ we see that $a_{0}^{-1} X$ divides over $M_{0}$ so over $\emptyset$.

Section 7 of this paper is devoted to establishing the uniqueness of invariant (under the group action) measures for definable groups with $f_{s g}$ (assuming NIP). In the case of generically stable groups this can be seen quickly and we do it now. 
Lemma 6.8. Suppose the definable group $G$ is generically stable, and $\mu$ is a left invariant global Keisler measure on $G$. Then $\mu$ is generic, in the sense that for any definable subset $X$ of $G$, if $\mu(X)>0$ then $X$ is generic.

Proof. Suppose that $X$ is a nongeneric definable subset of $G$, defined over a model $M_{0}$. The argument in the proof of Remark 6.7 gives an $M_{0}$-indiscernible sequence $\left(a_{i}: i<\omega\right)$ such that $\left\{a_{i} X: i<\omega\right\}$ is inconsistent. But $\mu\left(a_{i} X\right)=\mu(X)$ for all $i$. By Lemma 2.8 of [16], $\mu(X)=0$.

Lemma 6.9. Suppose $G$ is a definable group with $f_{s} g^{+}$. Then any definable subset of $G$ is a Boolean combination of translates (cosets) of definable subgroups of $G$ of finite index and nongeneric definable sets.

Sketch of proof. Fix a formula $\phi(x, y) \in L$. By a $\phi$-formula we mean a Boolean combination of formulas $\phi(g x, c)(g \in G, c \in \bar{M})$. By a global $\phi$-type of $G$ we mean a maximal consistent set of $\phi$-formulas over $\bar{M}$ (containing in addition $x \in G$ ). A global $\phi$-type will be called generic iff it contains only generic formulas (iff it extends to a global generic type of $G)$. As in the proof of 6.6, there is a definable subgroup $G_{\phi}^{0}$ of finite index which is contained in the (say left) stabilizer of every global generic $\phi$-type. It follows that each of the finitely many cosets of $G_{\phi}^{0}$ is consistent with a unique global generic $\phi$-type. Thus every $\phi$-set (set defined by a $\phi$-formula) is a Boolean combination of cosets of $G_{\phi}^{0}$ and nongeneric definable sets.

Corollary 6.10. Suppose the definable group $G$ is generically stable. Then there is a unique left invariant (under the group action) Keisler measure on $G$ which is also the unique right invariant Keisler measure.

Proof. If $\mu$ is a left invariant Keisler measure on $G$, then $\mu$ is determined on definable subgroups of finite index and their translates, and by Lemma 6.8 is 0 on all nongeneric sets. By Lemma 6.9 there is only one possibility for $\mu$. Lemma 6.9 is clearly still true replacing definable subgroups of finite index by normal definable subgroups of finite index. Hence we see that $\mu$ is also the unique right invariant Keisler measure on $G$.

Note that a special case is: if $G$ is a connected stable group, and $p$ is its unique global generic type, then not only is $p$ the unique left (right) invariant global type of $G$, but it is also the unique left (right) invariant global Keisler measure on $G$.

\section{Uniqueness of invariant measures for groups with $f s g$}

Given a definable group $G$ with $f s g$, and assuming NIP, we know from [16] that there is a left invariant global Keisler measure $\mu$ on $G$ (that is, $G$ is definably amenable). In fact we constructed such a $\mu$ which is generic (the definable sets of positive measure are precisely the generics). Clearly $\mu^{-1}$ is a right invariant generic global Keisler measure. Our uniqueness theorem (Theorem 7.7 below) generalizes the uniqueness of invariant types for connected stable groups, as well in a sense generalizing the uniqueness of Haar 
measure for compact groups. The main point is to prove a Fubini (or symmetry) theorem for suitable measures. Alessandro Berarducci also raised the uniqueness issue (in the $o$ minimal context) in [3] and pointed out the relevance of Fubini. Our methods go through variants of Grothendieck groups.

We begin with a lemma on homomorphisms from vector spaces to the reals. First recall some notation: if $A$ is a $\mathbb{Q}$-vector space, then a subset $P$ of $A$ is said to be a cone if $P$ is closed under addition and under scalar multiplication by positive elements of $\mathbb{Q}$.

Lemma 7.1. Let $A$ be a $\mathbb{Q}$-vector space, $B$ a $\mathbb{Q}$-subspace of $A, P$ a cone in $A$, and $\mu_{B}$ a nonzero homomorphism from $B$ to $\mathbb{R}$ with $\mu_{B}(B \cap P) \geq 0$. Assume that for any $a \in A$ there is $b \in B$ such that $b-a \in P$. Let $c \in A$ and let $e=\inf \left\{\mu_{B}(y): y \in B, y-c \in P\right\}$. Then:

(i) If e $=-\infty$ then there is no homomorphism $\mu: A \rightarrow \mathbb{R}$ extending $\mu_{B}$ with $\mu(P) \geq 0$.

(ii) If $e \in \mathbb{R}$, then there is a homomorphism $\mu: A \rightarrow \mathbb{R}$ extending $\mu_{B}$ with $\mu(P) \geq 0$ and $\mu(c)=e$.

Proof. First there is no harm in assuming that $0 \in P$.

(i) It is enough to prove the stronger statement that if $\mu: A \rightarrow \mathbb{R}$ is any homomorphism extending $\mu_{B}$, and $\mu(P) \geq 0$, then $\mu(c) \leq e$. But this is immediate: if $e^{\prime}>e$ then there is $y \in B$ such that $y-c \in P$ and $\mu_{B}(y) \leq e^{\prime}$; but then $\mu_{B}(y)-\mu(c) \geq 0$ so $\mu(c) \leq \mu_{B}(y) \leq e^{\prime}$.

(ii) Let us assume $e \in \mathbb{R}$, and by the stronger statement we have just proved all we need to do is to find a homomorphism $\mu: A \rightarrow \mathbb{R}$ extending $\mu_{B}$ such that $\mu(P) \geq 0$ and $\mu(c) \geq e$.

As $\mu_{B}$ is nonzero, let $b_{1} \in B$ be such that $\mu\left(b_{1}\right)>0$, and there is no harm in assuming $\mu_{B}\left(b_{1}\right)=1$. Replacing $c$ by $m b_{1}+c$ for sufficiently large $m$ we may also assume that $e>0$.

Let $P_{B}=\left\{b \in B: \mu_{B}(b) \geq 0\right\}$. Note that if $\mu: A \rightarrow \mathbb{R}$ is a homomorphism such that $\mu\left(b_{1}\right)=1$ and $\mu$ is nonnegative on $P_{B}$ then $\mu$ extends $\mu_{B}$. So it suffices to find some homomorphism $\mu$ from $A$ to $\mathbb{R}$ such that $\mu\left(b_{1}\right)=1, \mu$ is nonnegative on $P+P_{B}=\left\{a+b: a \in P, b \in P_{B}\right\}$, and $\mu(c) \geq e$. (Because then $\mu$ is nonnegative on $P_{B}$, and also on $P$.)

Let $P_{c}=\left\{\alpha c-\beta b_{1}: \alpha, \beta \in \mathbb{Q}, 0 \leq \beta<e \alpha\right\} \cup\{0\}$.

Claim. $-b_{1} \notin P^{\prime}=P+P_{B}+P_{c}$.

Proof of Claim. Suppose not.

Case (i): $-b_{1}=a+b+\alpha c-\beta b_{1}$ for $a \in P, b \in P_{B}$, and $\alpha, \beta$ as in the definition of $P_{c}$. Multiplying by $\alpha^{-1}$ and letting $a^{\prime}=\alpha^{-1} a, b^{\prime}=\alpha^{-1} b$, and $\gamma=\alpha^{-1} \beta$ we have $-\alpha^{-1} b_{1}=a^{\prime}+b^{\prime}+c-\gamma b_{1}$, whence $\gamma b_{1}-\left(b^{\prime}+\alpha^{-1} b_{1}\right)-c=a^{\prime} \in P$. But then $e \leq \mu_{B}\left(\gamma b_{1}-\left(b^{\prime}+\alpha^{-1} b_{1}\right)\right) \leq \gamma<e$, a contradiction.

Case (ii): $-b_{1}=a+b$ for $a \in P, b \in P_{B}$. Then $-a=b_{1}+b$, so $-a \in B$ and $\mu_{B}(-a)>0$. But then also $a \in B$, so $a \in B \cap P$, so by assumption $\mu_{B}(a) \geq 0$, implying $\mu_{B}(-a)<0$, again a contradiction. 
By the claim we can extend the cone $P^{\prime}$ to a maximal cone $P^{\prime \prime}$ not containing $-b_{1}$, and note that $b_{1} \in P^{\prime \prime}$, and also both $P+P_{B}$ and $P_{c}$ are contained in $P^{\prime \prime}$. Note also that $P^{\prime \prime}$ defines a linear preorder on $A$, namely for each $d \in A, d \in P^{\prime \prime}$ or $-d \in P^{\prime \prime}$. (If $d \notin P^{\prime \prime}$ then $-b_{1} \in P^{\prime \prime}+\mathbb{Q}^{+} d$, so $-d \in P^{\prime \prime}+\mathbb{Q}^{+} b_{1}$, so $-d \in P^{\prime \prime}$.) Our assumptions on $A$ together with the definition of $P_{B}$ imply that for any $a \in A$ there is $n>0$ such that $n b_{1}-a \in P+P_{B}$, so also in $P^{\prime \prime}$. Thus $\mathbb{Q}^{+} b_{1}$ is cofinal in $A$. Now for $a \in A$, define $\mu(a)=$ $\inf \left\{q \in \mathbb{Q}: q b_{1}-a \in P^{\prime \prime}\right\}$. It is clear that $\mu: A \rightarrow \mathbb{R}$ is a homomorphism preserving the preorder, $\mu\left(b_{1}\right)=1$ and $\mu\left(P^{\prime \prime}\right) \geq 0$. Then $\mu\left(P+P_{B}\right) \geq 0$, as $P+P_{B} \subseteq P^{\prime \prime}$. But also, as $P_{c} \subseteq P^{\prime \prime}$, we have $c-\gamma b_{1} \in P^{\prime \prime}$ for all positive rational $\gamma<e$. Hence $\mu(c) \geq e$, and we have found the required $\mu$.

We now consider a certain variant of the Grothendieck (semi)group introduced in Section 5 of [16]. We will also work at the more general level of definable group actions rather than just definable groups. So we will fix a definable group action $(G, X)$ and a small model $M_{0}$ over which $(G, X)$ is definable. Using the notation analogous to that in [16], we will take the relevant semigroup $K_{\text {semi }}(X)$ to be the collection of nonnegative cycles $\sum_{i} k_{i} X_{i}$ in $X$ up to piecewise translation by members of $G\left(M_{0}\right) . K_{0}(X)$ will be the corresponding Grothendieck group. (Recall that we define $x_{1} \sim_{0} x_{2}$ for $x_{1}, x_{2} \in$ $K_{\text {semi }}(X)$ if there is $y \in K_{\text {semi }}(X)$ such that $x_{1}+y=x_{2}+y$ in $K_{\text {semi }}$. Then $K_{0}$ is the collection of $\sim_{0}$-classes together with formal inverses.) When we apply Lemma 7.1 to this situation, the $\mathbb{Q}$-vector space $A$ will be the tensor product of $\mathbb{Q}$ with $K_{0}(X)$. Define $P_{0}$ to be the image of $K_{\text {semi }}$ in $K_{0}$ (under the canonical map) and then $P$ will be $\{\alpha x$ : $\left.\alpha \in \mathbb{Q}^{+}, x \in P_{0}\right\}$, a cone in $A$. Define $B_{0}$ to be the image in $K_{0}(X)$ of the " $M_{0}$-definable" members of $K_{\text {semi }}(X)$, and then $B$ will be the tensor product of $\mathbb{Q}$ with $B_{0}$.

We now give a somewhat more concrete representation of the objects defined in the previous paragraph. It will be convenient both notationally and conceptually to introduce a certain category $\mathcal{D}$ in place of the semigroup of nonnegative cycles on $X$ (namely before identification via piecewise translations). First we think of $\mathbb{Z}$ as living in our monster model $\bar{M}$ as the directed union of the finite sets (or sorts) $\{-m, \ldots, m\}$. We also have the group structure on $\mathbb{Z}$ definable in $\bar{M}$, piecewise. Let $\tilde{X}$ be $X \times \mathbb{Z}$. A definable subset of $\tilde{X}$ is by definition a definable subset of $X \times\{-m, \ldots, m\}$ for some $m$. Likewise for $\tilde{G}=G \times \mathbb{Z}$, which is now a "definable" group. The natural action of $\tilde{G}$ on $\tilde{X}$ which we will call $*$ is also "definable", namely for each $m, n$ the map $(G \times\{-m, \ldots, m\}) \times(X \times$ $\{-n, \ldots,-n\}) \rightarrow(X \times\{-m-n, \ldots, m+n\})$ is definable. The objects of the category are definable subsets of $\tilde{X}$. If $Y, Y^{\prime}$ are in $\mathcal{D}$ then a morphism $f$ from $Y$ to $Y^{\prime}$ is an injective map $f$ from $Y$ into $Y^{\prime}$ such that there is a partition $Y=Y_{1} \cup \cdots \cup Y_{n}$ of $Y$ and elements $g_{i} \in \tilde{G}\left(M_{0}\right)=G\left(M_{0}\right) \times \mathbb{Z}$ such that for each $i$ and $y \in Y_{i}, f(y)=g_{i} * y$. Equivalently (by compactness) $f$ is a definable embedding of $Y$ in $Y^{\prime}$ such that for each $y \in Y$ there is $g \in$ $\tilde{G}\left(M_{0}\right)$ such that $f(y)=g * y$. So $Y$ and $Y^{\prime}$ will be isomorphic in $\mathcal{D}$ if there is $f$ as above which is a bijection between $Y$ and $Y^{\prime}$. Then as a set, $K_{\text {semi }}(X)$ is the set of isomorphism classes of members of $\mathcal{D}$. The addition on $K_{\text {semi }}(X)$ is induced by the following (nonwell defined) addition on $\mathcal{D}$ : if $Y, Y^{\prime}$ are definable subsets of $X \times\{-m, \ldots, m\}$ then $Y+Y^{\prime}=Y \cup\langle e, 2 m+1\rangle * Y^{\prime}$. (Likewise for $n Y$.) Connecting with earlier notation, $B_{0}$ is the image in $K_{0}(X)$ of the set of isomorphism classes of $M_{0}$-definable elements of $\mathcal{D}$ 
(and $B$ the tensor product of $B_{0}$ with $\mathbb{Q}$ ). For $Y$ a definable subset of $\tilde{X},[Y]$ denotes its image in $A=K_{0}(X) \otimes \mathbb{Q}$.

We will be interested in Keisler measures $\mu$ on $X$ which are $G\left(M_{0}\right)$-invariant. Note that any such $\mu$ extends uniquely to (and is determined by) a $\tilde{G}_{0}$-invariant finitely additive measure on the definable subsets of $\tilde{X}$.

Lemma 7.2. The global $G\left(M_{0}\right)$-invariant Keisler measures on $X$ correspond to the homomorphisms $h: A \rightarrow \mathbb{R}$ such that $h(P) \geq 0$ and $h[X]=1$. Namely if $\mu$ is a global $G\left(M_{0}\right)$-invariant Keisler measure on $X$, then there is a unique homomorphism $h: A \rightarrow \mathbb{R}$ such that $h([Y])=\mu(Y)$ for any definable subset $Y$ of $X$. Moreover this $h$ satisfies $h([X])=1$ and $h(P) \geq 0$. Conversely if $h: A \rightarrow \mathbb{R}$ is such that $h([X])=1$ and $h(P) \geq 0$ then defining $\mu(Y)=h([Y])$ for any definable subset $Y$ of $X$ gives a global $G\left(M_{0}\right)$-invariant Keisler measure $\mu$ on $X$.

Proof. Clear.

Corollary 7.3. Suppose that $\mu$ is a global $G\left(M_{0}\right)$-invariant Keisler measure on $X$ which is moreover the unique such measure on $X$ extending $\mu \mid M_{0}$. Then for any definable subset $D$ of $X($ or $\tilde{X})$ with $\mu(D)=\beta$ and $\epsilon>0$, there are $n \in \mathbb{N}, M_{0}$-definable $E_{0}, E_{1} \in \mathcal{D}$ and $D^{\prime} \in \mathcal{D}$ such that:

(i) $\mu\left(E_{1}\right)-\mu\left(E_{0}\right)<n(\beta+\epsilon)$,

(ii) there is a morphism $f$ (in D) from $E_{0}+n D+D^{\prime}$ to $E_{1}+D^{\prime}$.

Proof. With earlier notation let $h: A \rightarrow \mathbb{R}$ be the homomorphism corresponding to $\mu$ as given by Lemma 7.2. So by Lemma 7.2, $h$ is the unique homomorphism such that $h(P) \geq 0$, and which extends $h \mid B$. Let $D$ be a definable subset of $X$ (or $\tilde{X}$ ) and put $c=[D]$. Then by Lemma 7.1, $\mu(D)=h([D])=\inf \{h(y): y \in B, y-c \in P\}$. Fix $\epsilon>0$. So for some $y \in B$ we have $h(y)<\mu(D)+\epsilon$ and $y-c \in P$. Now for large enough positive $n$ we have $n(y-c)=\left[D^{\prime \prime}\right]$ for some definable subset $D^{\prime \prime}$ (of $\tilde{X}$ ), and $n y=\left[E_{1}\right]-\left[E_{0}\right]$ with $E_{0}, E_{1} M_{0}$-definable subsets of $\tilde{X}$. So we have

(a) $\mu\left(E_{1}\right)-\mu\left(E_{0}\right)=h\left(\left[E_{1}\right]-\left[E_{0}\right]\right)=n h(y)<n(\mu(D)+\epsilon)$,

which gives (i). We also have

(b) $\left[E_{1}\right]-\left[E_{0}\right]-n[D]=\left[D^{\prime \prime}\right]$.

We then obtain some definable $D^{\prime}$ such that in $\mathcal{D}, E_{0}+D^{\prime \prime}+n D+D^{\prime}$ is isomorphic to $E_{1}+D^{\prime}$. Ignoring $D^{\prime \prime}$ this gives a morphism $f$ in $\mathcal{D}$ from $E_{0}+n D+D^{\prime}$ to $E_{1}+D^{\prime}$, which is (ii).

Let us give a more explicit statement.

Remark 7.4. The conclusion of Corollary 7.3 can be restated as: For any definable subset $D$ of $X$ with $\mu(D)=\beta$ and for any $\epsilon>0$ there are $n, m, m^{\prime}, m^{\prime \prime} \in \mathbb{N}, M_{0^{-}}$ definable sets $E_{0}, E_{1}$ in $\mathcal{D}$, and some $D^{\prime} \in \mathcal{D}$, as well as $D_{i} \in \mathcal{D}$ and $g_{i} \in \tilde{G}_{0}\left(M_{0}\right)$ for $i=1, \ldots, m, D_{j}^{\prime} \in \mathcal{D}$ and $g_{j}^{\prime} \in \tilde{G}_{0}\left(M_{0}\right)$ for $j=1, \ldots, m^{\prime}$, and $D_{k}^{\prime \prime} \in \mathcal{D}$ and $g_{k}^{\prime \prime} \in \tilde{G}_{0}\left(M_{0}\right)$ for $k=1, \ldots, m^{\prime \prime}$ such that: 
(i) $\mu\left(E_{1}\right)-\mu\left(E_{0}\right)<n(\beta+\epsilon)$,

(ii) $n D=\bigcup_{i=1}^{m} D_{i}, D^{\prime}=\bigcup_{j=1}^{m^{\prime}} D_{j}^{\prime}, E_{0}=\bigcup_{k=1}^{m^{\prime \prime}} D_{k}^{\prime \prime}$, the $D_{i}, D_{j}^{\prime}, D_{k}^{\prime \prime}$ are pairwise disjoint, and $D^{\prime}$ is disjoint from both $D$ and $E_{1}$,

(iii) the sets $g_{i} D_{i}, g_{j}^{\prime} D_{j}^{\prime}$ and $g_{k}^{\prime \prime} D_{k}^{\prime \prime}$ are pairwise disjoint subsets of $E_{1} \cup D^{\prime}$.

We can now prove the desired symmetry (or Fubini) theorem. Recall that if $\mu_{x}, \lambda_{y}$ are global Keisler measures on definable sets $X, Y$ respectively, and $\mu$ is definable (or even Borel definable), then we can form the global Keisler measure $\mu \otimes \lambda$ on $X \times Y$ : for $D$ a definable subset of $X \times Y,(\mu \otimes \lambda)(D)=\int \mu(D(y)) d \lambda$, where $D(y)=\{x:(x, y) \in D\}$. We may also write $(\mu \otimes \lambda)(D)$ as $\int_{D} d \mu d \lambda$.

Proposition 7.5. Suppose $(G, X)$ is a definable group action, and $Y$ a definable set, all defined over $M_{0}$. Suppose $\mu$ is a global Keisler measure on $X$ which is definable and satisfies the hypothesis of Corollary 7.3. Suppose $\lambda$ is a global definable Keisler measure on $Y$. Then $\mu_{x} \otimes \lambda_{y}=\lambda_{y} \otimes \mu_{x}$.

Before beginning the proof, let us define an action of $G$ on $X \times Y$ by $g(x, y)=(g x, y)$. We claim that both measures $\mu \otimes \lambda$ and $\lambda \otimes \mu$ are $G\left(M_{0}\right)$-invariant for this action: Let $D$ be a definable subset of $X \times Y$, and $g \in G\left(M_{0}\right)$. So for any $y \in Y,(g D)(y)=g(D(y))$, so $\mu((g D)(y))=\mu(D(y))$, and thus $(\mu \otimes \lambda)(g D)=(\mu \otimes \lambda)(D)$. On the other hand, let $f(x)=\lambda(D(x))$. Now clearly $D\left(g^{-1} x\right)=(g D)(x)$, so $f\left(g^{-1} x\right)=\lambda(g D(x))$. As $\mu$ is $G\left(M_{0}\right)$-invariant, $\int f(x) d \mu=\int f\left(g^{-1} x\right) d \mu$, so $(\lambda \otimes \mu)(D)=(\lambda \otimes \mu)(g D)$. All this of course extends to the actions of $\tilde{G}$ on $\tilde{X}$ and $\tilde{X} \times Y$ and the relevant measures on $\mathcal{D}_{X}$ and $\mathcal{D}_{X \times Y}$.

Proof of Proposition 7.5. Let $D$ be a definable subset of $X \times Y$. We have to show that $(\mu \otimes \lambda)(D)=(\lambda \otimes \mu)(D)$. By considering also the complement of $D$, it suffices to prove that for any $\epsilon>0,(\lambda \otimes \mu)(D) \leq(\mu \otimes \lambda)(D)+\epsilon$.

Fix $\epsilon>0$. By Corollary 7.3, for each $y \in Y$ we find $n_{y}, m_{y}, m_{y}^{\prime}, m_{y}^{\prime \prime},\left(E_{0}\right)_{y},\left(E_{1}\right)_{y}$, $D_{y}^{\prime}$ etc. such that (i), (ii) and (iii) of Remark 7.4 hold for $D_{y}=\{x:(x, y) \in D\}$. By compactness we may partition $Y$ into definable sets $Y_{v}$ such that the $n_{y}, m_{y}, m_{y}^{\prime}, m_{y}^{\prime \prime}$, $\left(E_{0}\right)_{y},\left(E_{1}\right)_{y},\left(g_{i}\right)_{y},\left(g_{j}^{\prime}\right)_{y},\left(g_{k}^{\prime \prime}\right)_{y}$ are constant on each $Y_{v}$. Focus attention on one $Y_{v}$. Let $D_{v}=D \cap\left(X \times Y_{v}\right)$. Let $D_{v}^{\prime} \subseteq X \times Y_{v}$ be such that for $y \in Y_{v}, D_{y}^{\prime}=\left\{x:(x, y) \in D_{v}^{\prime}\right\}$. So clearly we have:

Claim I. There is a morphism in $\mathcal{D}_{X \times Y_{v}}$ from $\left(E_{0} \times Y_{v}\right)+n D_{v}+D_{v}^{\prime}$ into $\left(E_{1} \times Y_{v}\right)+D_{v}^{\prime}$.

As $\mu\left(E_{1}\right)-\mu\left(E_{0}\right)<n\left(\mu\left(D_{y}\right)+\epsilon\right)$ for all $y \in Y_{\nu}$, we obtain, on integrating with respect to $\lambda$ over $Y_{v}$ :

Claim II. $\left(\mu\left(E_{1}\right)-\mu\left(E_{0}\right)\right) \lambda\left(Y_{v}\right) \leq n(\mu \otimes \lambda)\left(D_{v}\right)+\epsilon \lambda\left(Y_{v}\right)$.

But the left hand side in Claim II coincides with $(\lambda \otimes \mu)\left(E_{1} \times Y_{v}\right)-(\lambda \otimes \mu)\left(E_{0} \times Y_{v}\right)$. So denoting $\lambda \otimes \mu$ by $r$ we rewrite Claim II as

Claim III. $r\left(E_{1} \times Y_{v}\right)-r\left(E_{0} \times Y_{v}\right) \leq n(\mu \otimes \lambda)\left(D_{v}\right)+\epsilon \lambda\left(Y_{v}\right)$. 
We have already noted that $r$ on $X \times Y$ is $G\left(M_{0}\right)$-invariant, so applying $r$ to Claim I and using the disjointness there, we obtain

Claim IV. $r\left(E_{0} \times Y_{v}\right)+n r\left(D_{v}\right)+r\left(D_{v}^{\prime}\right) \leq r\left(E_{1} \times Y_{v}\right)+r\left(D_{v}^{\prime}\right)$.

From Claims II and IV we obtain

Claim V. $r\left(D_{v}\right) \leq(\mu \otimes \lambda)\left(D_{v}\right)+\epsilon \lambda\left(Y_{v}\right)$.

Summing over $v$ in Claim V, we obtain $(\lambda \otimes \mu)(D) \leq(\mu \otimes \lambda)(D)+\epsilon$, which is what we wanted. The proposition is proved.

Lemma 7.6. (Assume NIP.) Suppose G is a definable group, defined over a small model $M_{0}$, and $\mu$ is a Keisler measure on $G$ over $M_{0}$, which is left $G\left(M_{0}\right)$-invariant. Then:

(i) There is a global left $G\left(M_{0}\right)$-invariant Keisler measure $\mu^{\prime}$ extending $\mu$, and a small model $M$ containing $M_{0}$ such that $\mu^{\prime}$ is the unique left $G\left(M_{0}\right)$-invariant global Keisler measure extending $\mu^{\prime} \mid M$. Again any such $\mu^{\prime}$ is definable.

(ii) Suppose in addition that $G$ has $f s g$. Then any $\mu^{\prime}$ as in (i) is left invariant, namely left $G(\bar{M})$-invariant.

Proof. (i) is proved by the same method as 5.8, although our hypothesis here is weaker. Namely if the conclusion fails, we construct an increasing sequence $M_{\alpha}$ of models, and Keisler measures $\mu_{\alpha}$ over $M_{\alpha}$ on $G$, each $G\left(M_{0}\right)$-invariant, and such that for each $\alpha$ there is a subset $X_{\alpha}$ of $G$ definable over $M_{\alpha+1}$ and $r>0$ such that $\mu_{\alpha+1}\left(X_{\alpha} \triangle Y\right)>r$ for all $M_{\alpha}$-definable subsets $Y$ of $G$. Similar to the proof of 5.8, this construction depends on the fact, given either by compactness or by Construction $(*)$ of [16], that any $G\left(M_{0}\right)$ invariant Keisler measure over a model $M \supseteq M_{0}$ extends to a global $G\left(M_{0}\right)$-invariant Keisler measure. In any case we get a contradiction to NIP as in 5.8.

(ii) By the definability of $\mu^{\prime}, H=\operatorname{Stab}\left(\mu^{\prime}\right)$ is a type-definable subgroup of $G$. We want to show that it is all of $G$. If not, it is clear that the complement of $H$ contains a generic definable subset $X$ of $G$. By the $f s g, X \cap G\left(M_{0}\right)$ is nonempty. But then $X$ contains an element of $H$ (as $\mu^{\prime}$ is $G\left(M_{0}\right)$-invariant, whence $\left.G\left(M_{0}\right) \subseteq H\right)$. Contradiction.

Combining the above with the material of the previous section we can now obtain our main result.

Theorem 7.7. (Assume NIP.) Suppose $G$ is a definable group with $f s g$. Then $G$ has a unique left invariant global Keisler measure, which is also the unique right invariant global Keisler measure of $G$. This measure is both definable and generic.

Proof. We already know from [16] that $G$ has some left invariant global Keisler measure. Let $\mu, \lambda$ be left invariant global Keisler measures. We will first show that $\mu=\lambda^{-1}$. Let $D$ be any definable subset of $G$. We want to prove that $\mu(D)=\lambda\left(D^{-1}\right)$. Let $M_{0}$ be a small model over which both $G$ and $D$ are defined. Let $\mu^{\prime \prime}$ be a global Keisler measure satisfying (i) of 7.6 for some small $M$ containing $M_{0}$. That is, $\mu^{\prime \prime}$ extends $\mu \mid M_{0}$ and is the unique left $G\left(M_{0}\right)$-invariant Keisler measure extending $\mu^{\prime \prime} \mid M$. By (ii) of 7.6, $\mu^{\prime \prime}$ is left $G(\bar{M})$-invariant, in particular left $G(M)$-invariant, so is also the unique left $G(M)$ invariant extension of $\mu^{\prime \prime} \mid M$. So if we rename $M$ as $M_{0}, \mu^{\prime \prime}$ satisfies the hypothesis of 7.3. 
By Lemma 7.6, $\mu^{\prime \prime}$ is definable, and as already mentioned, $\mu^{\prime \prime}$ is left invariant. So as we are currently just interested in $\mu(D)$ we may assume that $\mu=\mu^{\prime \prime}$. Likewise we may assume that $\lambda$ is definable. By Proposition 7.5, $\mu \otimes \lambda=\lambda \otimes \mu$ (on $G \times G$ ). Let $Z=\{(x, y) \in G \times G: x \in y D\}$, which is equal to $\left\{(x, y) \in G \times G: y \in x D^{-1}\right\}$. So $(\mu \otimes \lambda)(Z)=\int \mu(y D) d \lambda=\mu(D)$ as $\mu$ is left invariant. Likewise $(\lambda \otimes \mu)(Z)=$ $\int \lambda\left(x D^{-1}\right) d \mu=\lambda\left(D^{-1}\right)$.

So we have succeeded in proving that $\lambda=\mu^{-1}$. This applies in particular when $\lambda=\mu$, showing that $\mu=\mu^{-1}$ (so $\mu$ is also right invariant). Hence $\mu=\lambda$ and is also the unique right invariant global Keisler measure on $G$.

Now we point out an extension of Proposition 7.7 to homogeneous spaces.

Proposition 7.8. (Assume NIP.) Suppose $(G, X)$ is a definable homogeneous space, and $G$ has $f s g$. Then there is a unique G-invariant global Keisler measure on $X$.

Proof. Let $\mu$ be the unique invariant global Keisler measure on $G$ given by the previous proposition. So it is definable and satisfies the hypothesis of 7.3. Note that we obtain from $\mu$ a $G$-invariant global Keisler measure $\lambda$ on $X$ : given $x \in X$, the map taking $g \in G$ to $g x$ gives a $G$-invariant surjection $\pi_{x}: G \rightarrow X$. For $Y$ a definable subset of $X$, define $\lambda_{x}(Y)=\mu\left(\pi_{x}^{-1}(Y)\right)$. Then $\lambda_{x}$ is $G$-invariant and does not depend on the choice of $x$ so we call it $\lambda$. Note that for any $x \in X$, and definable subset $Y$ of $X$, $\lambda(Y)=\mu(\{g \in G: g x \in Y\})$. Clearly $\lambda$ is also definable and satisfies the hypothesis of 7.3.

Let $\lambda^{\prime}$ be another $G$-invariant global Keisler measure on $X$. We want to show $\lambda^{\prime}=\lambda$. As in the proof of 7.7 we may assume $\lambda^{\prime}$ is definable. By 7.5, $\mu \otimes \lambda^{\prime}=\lambda^{\prime} \otimes \mu$ on $G \times X$. Let $Y$ be a definable subset of $X$ and let $Z=\{(g, x): g x \in Y\}$. Then $\left(\mu \otimes \lambda^{\prime}\right)(Z)=$ $\int_{x \in X} \mu(\{g \in G: g x \in Y\}) d \lambda^{\prime}=\int_{x \in X} \lambda(Y) d \lambda^{\prime}=\lambda(Y)$.

And $\left(\lambda^{\prime} \otimes \mu\right)(Z)=\int_{g \in G} \lambda^{\prime}(\{x \in X: g x \in Y\}) d \mu=\int_{g \in G} \lambda^{\prime}(Y) d \mu=\lambda^{\prime}(Y)$. So $\lambda=\lambda^{\prime}$

The remainder of this section is concerned with some elaborations on the material above, as well as some speculations and questions. There is an obvious formulation of $X$ having $f s g$ where $X$ is a definable homogeneous space (for a definable group $G$ ): there is a global type $p$ of $X$ such that for every $g \in G, g p$ is finitely satisfiable in $M_{0}$ for every model $M_{0}$.

If $G$ has $f s g$ then clearly $X$ does too.

Question 7.9. (Assume NIP.)

(i) Does Proposition 7.8 hold if only $X$ has $f s g$ ?

(ii) Are there examples of transitive group actions $(G, X)$ such that $X$ has $f s g, G$ acts faithfully, and $G$ does not have $f$ s $g$ ?

Remark 7.10. Let us say that a definable group $G$ has the weak $f s g$ if there is some small model $M_{0}$ over which $G$ is defined such that $G$ has no proper type-definable subgroup containing $G\left(M_{0}\right)$. The following results follow from the proofs above. 
(i) (Assume NIP.) If $G$ is definably amenable with weak $f_{s} g$ then $G$ has a unique left invariant Keisler measure which is also its unique right invariant Keisler measure, and is definable.

(ii) (Assume NIP.) Suppose the definable group $G$ is definably amenable and has no proper nontrivial type-definable subgroups. Then $G$ has a global left invariant type, which is definable, and is moreover the unique left invariant Keisler measure and the unique right invariant Keisler measure on $G$.

Proof. (i) is given by Proposition 7.5, and the proofs of Lemma 7.6 and Theorem 7.7.

The assumptions of (ii) imply that $G$ has weak $f s g$, and so let $\mu$ be the unique (left, right) invariant measure on $G$ given by (i). As $\mu$ is definable, $\mu$ does not fork over some small $M_{0}$. Let $p$ be some random global type for $\mu$, that is, every formula in $p$ has positive $\mu$-measure. Then clearly no left translate of $p$ forks over $M_{0}$. By Proposition 5.6, $\operatorname{Stab}_{l}(p)=G^{00}$. But our assumptions imply that $G^{00}=G$, hence $p$ is left invariant. By part (i), $\mu=p$.

Note that statement (ii) in Remark 7.10 above mentions only types, but the only proof we have of it involves measures. In fact, using 7.5 one can conclude that, assuming NIP, any group with weak $f s g$ has $f s g$ (and so the groups in Remark 7.10(ii) are generically stable groups in the sense of Section 6) but the proof uses the theory of generically stable measures which will be treated in a future paper.

Now we give a tentative definition of the $f s g$ property for a complete type over a small set, rather than a definable group. For simplicity we make a blanket assumption of NIP.

Definition 7.11. We say that $p(x) \in S(A)$ has $f_{s} g$ if $p$ has a global extension $p^{\prime}(x)$ such that for every $(|L|+|A|)^{+}$-saturated model $M$ containing $A, p^{\prime}$ is finitely satisfiable in $p(M)$.

Remark 7.12. (i) In Definition 7.11 the global extension $p^{\prime}$ of $p$ is necessarily a nonforking extension of $p$.

(ii) Suppose $A=\operatorname{bdd}(A)$ and $p(x) \in S(A)$ has $f s g$. Then $p$ has a unique global nonforking extension $p^{\prime}$, and $p^{\prime}$ is generically stable.

(iii) Suppose the global type $p^{\prime}$ is generically stable and $p^{\prime}$ does not fork over $A$. Let $p=p^{\prime} \mid A$. Then $p$ has $f s g$.

Proof. (i) If $\left(b_{i}: i<\omega\right)$ is an $A$-indiscernible sequence (in $\bar{M}$ ) then we can find a $(|L|+|A|)^{+}$-saturated model $M$ containing $A$ such that $\left(b_{i}: i<\omega\right)$ remains indiscernible over $M$. If $\phi\left(x, b_{0}\right) \in p^{\prime}$ then $\models \phi\left(a, b_{0}\right)$ for some $a \in M$, hence $\phi\left(a, b_{i}\right)$ for all $i<\omega$. So $p^{\prime}$ does not fork over $A$.

(ii) By part (i) and 2.11 (and the assumption that $A=\operatorname{bdd}(A)), p^{\prime}$ is $A$-invariant. We will show that some (any) Morley sequence in $p^{\prime}$ over $A$ is totally indiscernible (and apply 3.2). Let $M$ be a small $(|L|+|A|)^{+}$-saturated model containing $A$. Let $\left(a_{i}: i<\omega\right)$ be a Morley sequence in $p^{\prime}$ over $M$ (so also one over $A$ ). It is enough to prove that for any $n$, $\operatorname{tp}\left(a_{0}, \ldots, a_{n-1}, a_{n} / A\right)=\operatorname{tp}\left(a_{n}, a_{0}, \ldots, a_{n-1} / A\right)$. Suppose not, and let $\phi\left(x_{0}, \ldots, x_{n}\right)$ be a formula over $A$ such that $\models \phi\left(a_{0}, \ldots, a_{n-1}, a_{n}\right) \wedge \neg \phi\left(a_{n}, a_{0}, \ldots, a_{n-1}\right)$. So

(a) $\phi\left(x_{0}, \ldots, x_{n-1}, x_{n}\right) \in p^{\prime(n+1)}\left(x_{0}, \ldots, x_{n}\right) \mid A$, but

(b) $\neg \phi\left(x, a_{0}, \ldots, a_{n-1}\right) \in p^{\prime} \mid\left(A, a_{0}, \ldots, a_{n-1}\right)$. 
By (b) and our assumptions, $\neg \phi\left(x, a_{0}, \ldots, a_{n-1}\right)$ is realized by some $c \in M$ which realizes $p$. But then $\left(c, a_{0}, a_{1}, \ldots, a_{n-1}\right)$ also begins a Morley sequence in $p^{\prime}$ over $A$, so by $(\mathrm{a}),=\phi\left(c, a_{0}, \ldots, a_{n-1}\right)$, giving a contradiction.

(iii) follows from the material in Section 3 and is left to the reader.

In the context of Remark 7.12(iii) we say that the $f s g$ type $p(x) \in S(A)$ comes from a generically stable type. So (by 7.12(ii)) group actions, namely the action of the compact Lascar group $\operatorname{Aut}(\operatorname{bdd}(A) / A)$ on the set of extensions of $p$ over $\operatorname{bdd}(A)$, will enter the picture whenever an $f$ s $g$ type $p(x) \in S(A)$ does not come from a generically stable type.

Another source of $f s g$ types is through transitive group actions and construction $\mathrm{C} 2$ from Section 1 . Namely suppose that $(G, X)$ is a $\emptyset$-definable group action in the model $M$ of the NIP theory $T$, and we form $M^{\prime \prime}$ as in construction C2 with additional sort $S$. Then in $T^{\prime \prime}=\operatorname{Th}\left(M^{\prime \prime}\right)$ there is a unique (so isolated) 1-type $p(x)$ over $\emptyset$ in $S$. If $X$ has $f_{s} g$ in $T$ then it is not hard to see that $p$ has $f s g$ in $T^{\prime \prime}$.

In fact, types $p \in S(A)$ with $f s g$ can be characterized as types of the form $\mu \mid A$ where $\mu$ is a global $A$-invariant generically stable measure (in the sense of Remark 4.4). This was proved by P. Simon and will again appear in a joint work with the authors.

\section{The Compact Domination Conjecture}

We will prove

Theorem 8.1. Assume $\bar{M}$ is an o-minimal expansion of a real closed field. Let $G$ be a definably connected definably compact commutative group definable in $\bar{M}$. Then $G$ is compactly dominated. That is, let $\pi: G \rightarrow G / G^{00}$ be the canonical surjective homomorphism. Then for any definable subset $X$ of $G, Y_{X}=\left\{b \in G / G^{00}: \pi^{-1}(b) \cap X \neq \emptyset\right.$ and $\left.\pi^{-1}(b) \cap G \backslash X \neq \emptyset\right\}$ has Haar measure 0 .

In fact the proof (and subsequent elaborations) will yield a bit more: in the structure $\bar{M}^{*}$ obtained by expanding $\bar{M}$ by a predicate for $G^{00}, G / G^{00}$ will be "semi-o-minimal" with dimension that of $G$, and moreover for any definable subset $X$ of $G$ the set $Y_{X}$ above (which is now definable in $\bar{M}^{*}$ ) has dimension $<\operatorname{dim}\left(G / G^{00}\right)$. So in a sense $G$ is $o$-minimally dominated (by a definable $o$-minimal compact Lie group). This of course suggests many problems and issues for future work. Also, in the paper [17] joint with Peterzil, the full compact domination conjecture (i.e. for not necessarily commutative definably compact groups) is deduced from Theorem 8.1 and results in [16], using a structure theorem for definably compact groups in $o$-minimal expansions of real closed fields. Of course Theorem 8.1 builds on and uses the work and contributions of a number of people, including Berarducci, Dolich, Edmundo, Otero, and Peterzil.

Until we say otherwise, $\bar{M}$ denotes a (saturated) $o$-minimal expansion of a real closed field $K, G$ is a definably compact definably connected definable group in $\bar{M}$ of $o$-minimal dimension $n$, and $\pi$ is the canonical surjective homomorphism from $G$ to $G / G^{00}$. Without loss of generality $G$ is defined over $\emptyset$. $M_{0}$ will denote a fixed small submodel of $\bar{M}$. We will make use in a few places of the fact that $G^{00}$ can be defined by a countable collection of formulas. (This is by the DCC result in [4].) By [16], $G / G^{00}$ with its logic topology 
is a connected compact commutative Lie group of dimension $n$. We will feel free to use this below. The overall proof of 8.1 has several steps and "new" ingredients, including a beautiful result of Otero and Peterzil ([25]). Some of the lemmas go through or can be formulated at various greater levels of generality but we tend to concentrate on the case at hand. By Lemma 10.5 of [16] in order to prove Theorem 8.1 it suffices to show that if $X$ is a definable subset of $G$ of dimension $<n$ then the Haar measure of $\pi(X) \subseteq G / G^{00}$ is 0 . We will aim towards this.

The first step is to show that $G^{00}$ is definable in some weakly $o$-minimal expansion of $\bar{M}$. Recall that weak $o$-minimality means that every definable set of elements (rather than tuples) of the universe is a finite union of convex sets (with respect to the underlying ordering). Let $\bar{M}^{*}$ be the expansion of $\bar{M}$ obtained by adjoining a predicate for the trace on $\bar{M}$ of any set definable with parameters in some elementary extension of $\bar{M}$. In other words, for each $L$-formula $\phi(x, y)(x, y$ tuples) and complete type $q(x)$ over $\bar{M}$, adjoin a predicate for $\{b: \phi(x, b) \in q(x)\}$. By the results of Baisalov and Poizat [2], or alternatively of Shelah [36], Th $\left(\bar{M}^{*}\right)$ is weakly $o$-minimal, and has quantifier elimination. (This will be used freely below.) The weak o-minimality of a theory means that every model is weakly $o$-minimal, namely that for some (any) model there is a bound on the number of convex components of definable subsets of 1-space in definable families.

Lemma 8.2. $G^{00}$ is definable in $\bar{M}^{*}$.

Proof. Let $p$ be a global generic type of $G$ (which exists by 5.2 and 5.3). By Proposition 5.6 (and 5.12) we have

(i) $\operatorname{Stab}(p)=G^{00}$,

and moreover as mentioned in the proof of 5.6,

(ii) $G^{00}=\left\{g_{1} g_{2}^{-1}: \operatorname{tp}\left(g_{1} / M_{0}\right)=\operatorname{tp}\left(g_{2} / M_{0}\right)\right\}$.

Given a formula $\psi(x)$ (over some parameters) defining a subset $X$ of $G$, let $\operatorname{Stab}_{\psi}(p)=$ $\{g \in G$ : for all $h \in G, h X \in p$ iff $g h X \in p\}$. Then clearly $\operatorname{Stab}_{\psi}(p)$ is a subgroup of $G$. $\operatorname{Moreover} \operatorname{Stab}(p)=\bigcap_{\psi} \operatorname{Stab}_{\psi}(p)$ and

(iii) $\operatorname{Stab}_{\psi}(p)$ is definable in $\bar{M}^{*}$.

We will show that $G^{00}$ is the intersection of finitely many $\operatorname{Stab}_{\psi}(p)$, even with the $\psi$ over $M_{0}$.

For $\psi(x)$ over $M_{0}$, let $S_{\psi}(p)$ be the smallest set type-definable over $M_{0}$ (in $\bar{M}$ ), containing $\operatorname{Stab}_{\psi}(p)$. (Note that as $p$ is $\operatorname{Aut}\left(\bar{M} / M_{0}\right)$-invariant, so is $\operatorname{Stab}_{\psi}(p)$.) We do not necessarily know that $S_{\psi}(p)$ is a subgroup of $G$, but:

(iv) $S_{\psi}(p) \cdot G^{00}$ is a type-definable subgroup of $G$ (clearly containing $\operatorname{Stab}_{\psi}(p)$ ).

Indeed, clearly $\pi\left(S_{\psi}(p)\right)$ is the closure of $\pi\left(\operatorname{Stab}_{\psi}(p)\right)$ in $G / G^{00}$, and hence is a closed subgroup of $G / G^{00}$. Its preimage in $G$ is thus a subgroup of $G$ and coincides with $S_{\psi}(p) \cdot G^{00}$.

(v) $\bigcap_{\psi}\left(S_{\psi}(p) \cdot G^{00}\right)=G^{00}$ (where the $\psi$ 's on the left hand side are taken only over $\left.M_{0}\right)$. 
Indeed, $\supseteq$ is obvious. Let us show the reverse inclusion. Fix $g \in G \backslash G^{00}$. So for every $h \in G^{00}, g h^{-1} \notin G^{00}$. So by (ii), for every $h \in G^{00}$ and $a$ realizing $p \mid M_{0}, g h^{-1} a$ does not realize $p \mid M_{0}$. By compactness there is a formula $\psi(x) \in p \mid M_{0}$ such that for any $h \in G^{00}$ and $a$ satisfying $\psi(x), g h^{-1} a$ does not satisfy $\psi(x)$. However, for any $g_{1} \in \operatorname{Stab}_{\psi}(p)$ clearly there is $a$ such that both $a$ and $g_{1} a$ satisfy $\psi$. Thus for every $g_{1} \in S_{\psi}(p)$ the same is true. So we have shown that (for our given choice of $g \notin G^{00}$ ), for all $h \in G^{00}, g h^{-1} \notin S_{\psi}(p)$. That is, $g \notin S_{\psi}(p) \cdot G^{00}$. This proves (v).

We can now complete the proof of the lemma. We know that $G$ has the DCC on typedefinable subgroups of bounded index (see [4]). Hence by (v), $G^{00}$ is the intersection of finitely many of the $S_{\psi}(p) \cdot G^{00}$. Thus (as $\left.G^{00} \subseteq \operatorname{Stab}_{\psi}(p) \subseteq S_{\psi}(p) \cdot G^{00}\right), G^{00}$ is the intersection of finitely many of the $\operatorname{Stab}_{\psi}(p)$. By (iii), $G^{00}$ is definable in $\bar{M}^{*}$.

Remark 8.3. (i) The proof above uses only the existence of an $f$-generic type (rather than a generic type), and hence, by 5.6 and 5.10, Lemma 8.2 goes through assuming only that $T$ has NIP, $G$ is a definable definably amenable group, and $G / G^{00}$ is a compact Lie group.

(ii) Otero and Peterzil pointed out to us a more direct proof of 8.2, but which makes more use of the fact that $G$ has $f s g$ : Choose a global generic type $p$ of $G$. By 4.3 of [16] and the fact that $G / G^{00}$ has DCC, $G^{00}$ is of the form $\operatorname{Stab}_{\mathcal{I}}\left(X_{1}\right) \cap \cdots \cap \operatorname{Stab}_{\mathcal{I}}\left(X_{n}\right)$, where the $X_{i}$ are definable sets in $p$, and $\mathcal{I}$ denotes the ideal of nongeneric definable subsets of $G$. (Here $\operatorname{Stab}_{\mathcal{I}}(X)$ is the set of $g \in G$ such that the symmetric difference of $X$ and $g X$ is nongeneric, and so is type-definable in $\bar{M}$.) But genericity (so also nongenericity) is "definable" in $\bar{M}^{*}$, because a definable subset $X$ of $G$ is generic iff for some $g \in G$, $g X \in p$. Hence each $\operatorname{Stab}_{\mathcal{I}}\left(X_{i}\right)$ is definable in $\mathcal{M}^{*}$, which suffices.

The next step is given to us by Lemma 4.3 of [25].

Lemma 8.4. Let I be the interval $[0,1)$ in $\bar{M}$ (or $K$ ). Then there are one-one definable continuous functions $\gamma_{1}, \ldots, \gamma_{n}$ from $I$ to $G$ such that $G=\gamma_{1}(I)+\cdots+\gamma_{n}(I)$ (where $\gamma_{j}(I)$ denotes the image of I under $\left.\gamma_{j}\right)$.

In fact the $\gamma_{i}$ are generators of the $o$-minimal fundamental group of $G$ and the proof in [25] has a (co)homological character.

For $j=1, \ldots, n$, let $I_{j}=\gamma_{j}(I)$. As $\pi$ is a surjective homomorphism we have:

Corollary 8.5. $G / G^{00}=\pi\left(I_{1}\right)+\cdots+\pi\left(I_{n}\right)$.

We are now in the following interesting situation. $G / G^{00}$ as well as its subsets $\pi\left(I_{j}\right)$ are compact spaces under the logic topology, namely in their capacity as bounded hyperdefinable sets in the saturated model $\bar{M}$, but they are also, by Lemma 8.2, definable (or rather interpretable) sets in the (nonsaturated) weakly $o$-minimal structure $\bar{M}^{*}$. We will show in the next step that each $\pi\left(I_{j}\right)$ is a "definable $o$-minimal set" in $\bar{M}^{*}$ which is piecewise an interval in $\mathbb{R}$.

Let us first be precise about what we mean by a definable set in an ambient structure being $o$-minimal. Let $N$ be a structure, and $X$ a definable (or interpretable) set in $N$. When we say "definable" we mean definable in $N$ with parameters from $N$. Suppose that 
$<$ is a definable linear ordering on $X$. We call $X$ o-minimal in $N$ with respect to $<$ if any definable subset $Y$ of $X$ is a finite union of intervals $(a, b)$ (where possibly $a=-\infty$, $b=+\infty)$ and singletons. We will call $X$ strongly o-minimal if in addition there is a finite bound on the number of intervals and points in definable families.

In the next proposition we will mention the existence of definable Skolem functions on a definable set. So let us again be precise about the meaning. Again let $N$ be a structure in language $L$ and $X$ a set definable (or interpretable) in $N$, for now definable without parameters. We say that $X$ has definable Skolem functions in $N$ (or the formula defining $X$ has definable Skolem functions in $\operatorname{Th}(N))$ if for any $L$-formula $\phi(x, y)$ where $x$ is a variable ranging over $X$, and $y$ is an arbitrary tuple of variables, there is a partial $\emptyset$ definable function $f_{\phi}(y)$ such that

$$
N \models(\forall y)\left((\exists x \in X \phi(x, y)) \rightarrow\left(\phi\left(f_{\phi}(y), y\right) \wedge f_{\phi}(y) \in X\right)\right) .
$$

We can also speak of $X$ having Skolem functions in $N$ over some set $A$ of parameters from $N$. The reader should note that if $X$ has definable Skolem functions in $N^{\text {eq }}$ (over some parameters), then $X$ has elimination of imaginaries (over some parameters), namely whenever $Z \subseteq X^{n}$ and an equivalence relation $E$ on $Z$ are definable in $N$ then there is a definable bijection of $Z / E$ with some definable $W \subseteq X^{m}$.

We now return to the main narrative, with notation following Lemma 8.4. The following proposition is fundamental. The appendix is devoted to the proof of part (iii).

Proposition 8.6. (Work in $\bar{M}^{*}$.) For each $j, \pi\left(I_{j}\right)$ is a finite disjoint union of definable sets $X_{1}, \ldots, X_{r}$ and points $c_{1}, \ldots, c_{s}$ such that each $X_{i}$ is equipped with a definable total ordering $<_{i}$ such that (for each $\left.i=1, \ldots, r\right)$ :

(i) $\left(X_{i},<_{i}\right)$ is (abstractly) isomorphic to $\mathbb{R}$ with the usual ordering,

(ii) $X_{i}$ is strongly o-minimal (with respect to $<_{i}$ ),

(iii) $X_{i}$ has definable Skolem functions after possibly naming some parameters from $\bar{M}^{*}$.

Proof. Let us fix $j$, and $X$ will denote $\pi\left(I_{j}\right)$. Note that $I_{j}$ has a canonical linear ordering

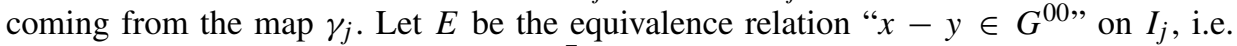
coming from $\pi$. As $E$ is definable in $\bar{M}^{*}$ (which has weakly $o$-minimal theory), each $E$-class is a union of at most $k$ convex sets for some $k$. Let $Y$ be the set of elements of $I_{j}$ which are in the "first" convex subset of their $E$-class. Then $Y$ is definable, so is a finite union of convex definable sets, and $\pi(Y)=\pi\left(I_{j}\right)=X$. Let $E_{Y}$ denote the restriction of $E$ to $Y$. Write $Y$ minimally as a finite disjoint union of (definable) convex sets $Y_{1}, \ldots, Y_{t}$. So each $E_{Y}$-class is convex and contained in a unique $Y_{i}$. Let $X_{i}=\pi\left(Y_{i}\right)$ and note that $<$ induces a linear ordering $<_{i}$ on $X_{i}$ (of course definable in $\bar{M}^{*}$ ). Let us restrict our attention to some $Y_{i}$ such that $X_{i}$ is infinite.

Claim (I). $<_{i}$ is dense on $X_{i}$ (i.e. if $a, b \in X_{i}$ and $a<_{i} b$ then there is $c \in X_{i}$ with $\left.a<_{i} c<_{i} b\right)$.

Proof of Claim (I). We may work inside an interval $I^{\prime}=\left(a^{\prime}, b^{\prime}\right)$ of $Y_{i}$ (i.e. with $\pi\left(a^{\prime}\right)$ $\leq_{i} a$ and $\left.\pi\left(b^{\prime}\right) \geq_{i} b\right)$. So $\pi^{-1}(a) \cap I^{\prime}$ and $\pi^{-1}(b) \cap I^{\prime}$ are convex sets which are typedefinable in $\bar{M}$ and disjoint. Moreover the first has no greatest element and the second has 
no smallest element (as every coset of $G^{00}$ is open in $G$, by Lemma 3.2 of [33]). Hence by compactness (in $\bar{M}$ ) there is $c^{\prime} \in I^{\prime}$ such that $\pi^{-1}(a) \cap I^{\prime}<c^{\prime}<\pi^{-1}(b) \cap I^{\prime}$. Let $c=\pi\left(c^{\prime}\right)$.

From Claim (I) we may assume that $\left(X_{i},<_{i}\right)$ has no first or last element (by removing them if they exist).

Claim (II). $\left(X_{i},<_{i}\right)$ is complete, that is, every subset bounded above (below) has a supremum (infimum).

Proof of Claim (II). Again we may work in $\pi\left(I^{\prime}\right) \subset X_{i}$ for some interval $I^{\prime}=\left(a^{\prime}, b^{\prime}\right)$ in $Y_{i}$. We consider $\pi\left(I^{\prime}\right)$ with the logic topology (equivalently as a closed subset of $\left.G / G^{00}\right)$. Let $(A, B)$ be a Dedekind cut in $\left(\pi\left(I^{\prime}\right),<_{i}\right)$. For $a \in A, A_{a}=\left\{x \in \pi\left(I^{\prime}\right)\right.$ : $\left.a \leq_{i} x\right\}$ is closed in $\pi\left(I^{\prime}\right)$, as it is clearly the image of a type-definable (in $\bar{M}$ ) subset of $I^{\prime}$. Likewise for $b \in B, B_{b}=\left\{x \in \pi\left(I^{\prime}\right): x \leq_{i} b\right\}$ is closed. Hence by compactness of the space $\pi\left(I^{\prime}\right)$, there is a point in the intersection of all the $A_{a}$ 's and $B_{b}$ 's. This suffices.

Before continuing with Claim (III), let us note that we can already deduce (ii) of the proposition. For if $Z$ is a definable (in $\bar{M}^{*}$ ) subset of $X_{i}$, then by weak $o$-minimality of $\bar{M}^{*}, \pi^{-1}(Z) \cap Y_{i}$ is a finite union of convex sets. By completeness of $\left(X_{i},<_{i}\right)$ (Claim (II) above), $Z$ is a finite union of intervals and points. Weak $o$-minimality of $\operatorname{Th}\left(\bar{M}^{*}\right)$ implies that there is a bound on the number of connected components of $Z$ as it varies in a definable family. So each $\left(X_{i},<_{i}\right)$ is strongly $o$-minimal (in the structure $\bar{M}^{*}$ ). We will use this in the proof of the next claim.

Claim (III). $\left(X_{i},<_{i}\right)$ is separable, namely has a countable dense subset with respect to the ordering $<_{i}$.

Proof of Claim (III). We know that $X$ with the logic topology is second countable (has a countable basis), because $E$ is given by countably many formulas. (See Remark 1.6 of [4] for example.) We will show that every $<_{i}$-interval $(a, b)$ in $X_{i}$ contains an open subset of $X$. So as $X$ has a countable basis, $\left(X_{i},<_{i}\right)$ will have a countable dense subset.

We work with one $i$ at a time. The reader should convince himself/herself that there is no harm in assuming that $Y=Y_{1}$. So $X=X_{1}$. We relabel $<_{1}$ as $<_{X}$. We fix an $<_{X}$-interval $(a, b)$ in $X$ and we want to find a subinterval which is open in $X$ (with the $\operatorname{logic}$ topology). Let $Z$ be the union of all the second convex components of $E$-classes in $I_{j}$. Let $E_{Z}$ be the restriction of $E$ to $Z$. So $Z$ is definable in $\bar{M}^{*}$ and is a disjoint union of finitely many definable convex subsets $Z_{1}, \ldots, Z_{m}$ of $I$ such that each $E_{Z}$-class is convex and contained in a unique $Z_{j}$. Consider $\pi(Z) \cap(a, b)$. If it is finite, then after passing to a smaller interval, we may assume that $\pi(Z) \cap[a, b]$ is empty. This means that for $c \in[a, b], \pi^{-1}(c) \subset Y$. Let $a_{0} \in \pi^{-1}(a) \cap I_{j}$ and $b_{0} \in \pi^{-1}(b) \cap I_{j}$. So $(a, b)$ is the set of $E$-classes which are contained in the interval $\left(a_{0}, b_{0}\right)$ in $I_{j}$. Thus $(a, b)$ is open in $X$ and we are finished.

So we may assume (by $o$-minimality of $\left(X,<_{X}\right)$ and passing to a smaller interval) that $(a, b)$ is contained in $\pi(Z)$. The ordering $<$ on $Z$ induces a definable ordering $<2$ on $\pi(Z) \subseteq X$. After ignoring finitely many points, we know (by the description of linear orderings definable on an $o$-minimal structure) that we can break up $(a, b)$ into finitely 
many $<_{X}$-intervals, on each of which $<_{2}$ agrees with $<_{X}$ or $>_{X}$. It follows that we can find a subinterval $(c, d)$ of $(a, b)$ and some $r=1, \ldots, m$ such that $\pi^{-1}([c, d]) \cap Z$ is contained in $Z_{r}$, and moreover for some $c_{0}^{\prime}, d_{0}^{\prime}$ in $Z_{r}$ (preimages of $c, d$ ), $[c, d]$ is the image under $\pi$ of the closed $<$-interval between $c_{0}^{\prime}$ and $d_{0}^{\prime}$, and moreover $<_{2}$ on $[c, d]$ agrees with $<_{X}$ or $>_{X}$.

Now if $k=2$ we are finished: Let $c_{0}, d_{0} \in Y$ be preimages of $c, d$. Then $(c, d)$ is precisely the set of $E$-classes contained in $\left(c_{0}, d_{0}\right) \cup\left(c_{0}^{\prime}, d_{0}^{\prime}\right)$ (or in $\left(c_{0}, d_{0}\right) \cup\left(d_{0}^{\prime}, c_{0}^{\prime}\right)$ if $<_{2}$ on $[c, d]$ is $>_{X}$ ).

If $k>2$ we continue, replacing $(a, b)$ by $(c, d)$, and considering now $W$ the union of the third convex components of $E$-classes. Our choice of $(c, d)$ means that passing to smaller subintervals does not disturb the compatibility with the second convex components of $E$-classes.

This finishes the proof of Claim (III).

It follows from Claims (I), (II) and (III) that $\left(X_{i},<_{i}\right)$ is isomorphic as an ordered set to $\mathbb{R}$ with its usual ordering. So we have proved (i).

We have already proved (ii). For (iii), see the appendix.

Let $X_{1}^{j}, \ldots, X_{r_{j}}^{j}$ be the sets obtained for $\pi\left(I_{j}\right)$ in Proposition 8.6. By Proposition 8.6, each $X_{k}^{j}$ is strongly $o$-minimal in $\bar{M}^{*}$. The reader should convince himself/herself that the general machinery of $o$-minimality (dimension, cell decomposition, etc.) applies to Cartesian products of the $X_{k}^{i}$ and definable (in $\bar{M}^{*}$ ) subsets $Z$ of such Cartesian products. In fact we will call such a definable set $Z$ a semi-o-minimal definable set. By Proposition 8.6(iii), and the discussion preceding the statement of 8.6, we have "elimination of imaginaries" for such definable sets: namely if $Z$ is a subset of a Cartesian product of the $X_{k}^{j}$ and $E$ an equivalence relation on $Z$, both definable in $\bar{M}^{*}$ then $Z / E$ is in definable bijection with some definable $W$ which is a subset of a Cartesian product of the $X_{k}^{j}$ s. We will apply this to $G / G^{00}$ considered as a group definable (or interpretable) in $\bar{M}^{*}$. In fact let us write $J$ for this group, so as to distinguish it from $G / G^{00}$ as a bounded hyperdefinable group in $\bar{M}$. By Corollary $8.5, J$ is in the definable closure (uniformly) of the $X_{k}^{j}$,s. Thus there is a definable subset $Z$ of some Cartesian product of the $X_{k}^{j}$, and a definable equivalence relation $E$ on $Z$ such that $J$ is definably isomorphic to $Z / E$. Hence $J$ is definably isomorphic to some definable $W$ which is a subset of a Cartesian product of the $X_{k}^{j}$, s. But $J$ also has a definable group structure, hence is a semi- $o$-minimal group. Note that $J$, being abstractly isomorphic to the connected commutative compact Lie group $G / G^{00}$, is divisible, and thus $J$ is definably connected in the sense of having no proper definable subgroups of finite index. The general theory ([31]) of equipping definable groups in $o$ minimal structures with a definable group manifold structure applies to $J$. We conclude using Proposition 8.6(i) that $J$ with its definable manifold topology is locally Euclidean, and thus (by the special case of Hilbert's 5th problem due to Pontryagin) is a connected commutative Lie group, whose Lie group dimension coincides with its semi- $o$-minimal dimension. By Corollary 8.5 the semi-o-minimal dimension of $J$ is at most $n$. On the other hand a connected commutative Lie group is a finite product of copies of $(\mathbb{R},+)$ 
and $S_{1}$. As $J$ is abstractly isomorphic to $G / G^{00}=S_{1}^{n}$, by considering torsion, it follows that, as a Lie group, $J$ has dimension $\geq n$. This yields

Corollary 8.7. J is a semi-o-minimal connected compact Lie group definable in $\bar{M}^{*}$ with both semi-o-minimal and Lie group dimension $n$.

We will point out later that, as expected, the topology on $J$ coincides with the logic topology on $G / G^{00}$. But we will be able to complete the proof of Theorem 8.1 without using this. The next step is:

Lemma 8.8. Let $Y$ be a definable (in $\bar{M}$ ) subset of $G$ of dimension $<n$. Then the semi$o$-minimal dimension of $\pi(Y) \subseteq J$ is $<n$.

Proof. The proof uses the ideas from the proof of Lemma 10.3 of [16], but with some additional complications. We first note that given $Y$ as in the hypothesis there is, using the fact that $\operatorname{Th}(\bar{M})$ has Skolem functions, a definable subset $Z$ of $I_{1} \times \cdots \times I_{n}$ such that the map $\left(x_{1}, \ldots, x_{n}\right) \mapsto x_{1}+\cdots+x_{n}$ induces a (definable) bijection between $Z$ and $Y$.

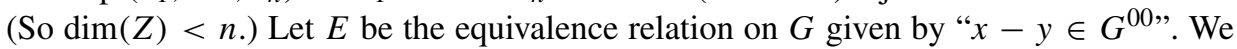
first prepare the scene. By 8.6 for each $j=1, \ldots, n, \pi\left(I_{j}\right)$ is a disjoint union of strongly $o$-minimal sets $\left(X_{i},<_{i}\right)$ in the sense of $\bar{M}^{*}$, so putting them together in some order, we view $\pi\left(I_{j}\right)$ as a strongly $o$-minimal set in $\bar{M}^{*}$, whose ordering may be written as just $<$ when there is no ambiguity. Furthermore, by the analysis at the beginning of the proof of 8.6, each $I_{j}$ is a finite disjoint union of convex subsets $Y_{j}^{i}$, say, each definable in $\bar{M}^{*}$, and such that all classes of the restriction of $E$ to $Y_{j}^{i}$ are convex. (Recall that we first considered the elements of $I_{j}$ which are in the "first" convex subset of their $E$-class and then wrote this set as a finite disjoint union of (definable) convex sets. Then consider the elements of $I_{j}$ in the "second" convex subset of their $E$-class, etc.) Note that the ordering we defined on $\pi\left(I_{j}\right)$ in 8.6 came from considering the induced ordering on the $E$-classes for suitable $Y_{j}^{i}$ (namely those consisting of elements of $I_{j}$ in the "first" convex subset of their $E$-class). Nevertheless we may assume that for each $Y_{j}^{i}$, the restriction of $\pi$ to $Y_{j}^{i}$ is an order preserving or reversing surjective map to an interval in $\pi\left(I_{j}\right)$ (using $o$-minimality of $\pi\left(I_{j}\right)$ in $\bar{M}^{*}$ as well as weak $o$-minimality of $\bar{M}^{*}$ ).

Let us denote by $\pi^{n}$ the natural map from $I_{1} \times \cdots \times I_{n}$ to $\pi\left(I_{1}\right) \times \cdots \times \pi\left(I_{n}\right)$. Recall that $Z$ was a definable (in $\bar{M}$ ) subset of $I_{1} \times \cdots \times I_{n}$ of dimension $<n$.

Claim. $\pi^{n}(Z)$ has dimension $<n$ in the semi-o-minimal structure $\pi\left(I_{1}\right) \times \cdots \times \pi\left(I_{n}\right)$ (in $\bar{M}^{*}$ ).

Proof of Claim. In fact we will prove, by induction on $n$, that if $W$ is any definable (in $\bar{M}$ ) subset of $I_{1} \times \cdots \times I_{n}$ of dimension $<n$, then $\pi^{n}(W) \subseteq \pi\left(I_{1}\right) \times \cdots \times \pi\left(I_{n}\right)$ has dimension $<n$. We may assume $W$ is the graph of a continuous definable function $f: C \rightarrow I_{n}$, where $C$ is an open definable subset of $I_{1} \times \cdots \times I_{n-1}$, and even that on $C, f$ is strictly monotone or constant in each coordinate. Assume for a contradiction that $\pi^{n}(W)$ has dimension $n$, in $\pi\left(I_{1}\right) \times \cdots \times \pi\left(I_{n}\right)$. It follows that $\pi^{n}(W)$ contains the closure of a subset of the form $U \times(a, b)$, where $U$ is an open rectangular box in $\pi\left(I_{1}\right) \times \cdots \times \pi\left(I_{n-1}\right)$ and $(a, b)$ is an interval in $\pi\left(I_{n}\right)$. Now $W$ is the disjoint union 
of its intersections with the various "rectangles" $Y_{1}^{i_{1}} \times \cdots \times Y_{n}^{i_{n}}$, where the $Y_{j}^{i}$ are as in the first paragraph. So by $o$-minimality of the $\pi\left(I_{j}\right)$ in $\bar{M}^{*}$ we can choose $i_{1}, \ldots, i_{n}$ such that if $W^{\prime}=W \cap\left(Y_{1}^{i_{1}} \times \cdots \times Y_{n}^{i_{n}}\right)$, then $\pi^{n}\left(W^{\prime}\right)$ contains the closure of a set $U \times(a, b)$ as above. Note that $W^{\prime}$ is now definable in $\bar{M}^{*}$ rather than $\bar{M}$ but it will not matter. Let $c \in \pi\left(I_{n}\right)$ be such that $a<c<b$. In fact by our assumptions on $Y_{n}^{i_{n}}, c$ will be in $\pi\left(Y_{n}^{i_{n}}\right)$. Lift $c$ to a point in $c^{\prime} \in Y_{n}^{i_{n}}$. Now let $x=\left(x_{1}, \ldots, x_{n}\right) \in U$. As both $(x, a)$ and $(x, b)$ are in $\pi\left(W^{\prime}\right)$, we can find $x^{\prime}=\left(x_{1}^{\prime}, \ldots, x_{n-1}^{\prime}\right)$ and $x^{\prime \prime}=\left(x_{1}^{\prime \prime}, \ldots, x_{n-1}^{\prime \prime}\right)$ in $C \cap\left(Y_{1}^{i_{1}} \times \cdots \times Y_{n-1}^{i_{n-1}}\right)$ such that $\pi^{n-1}\left(x^{\prime}\right)=\pi^{n-1}\left(x^{\prime \prime}\right)=x, \pi\left(f\left(x^{\prime}\right)\right)=a$ and $\pi\left(f\left(x^{\prime \prime}\right)\right)=b$, and of course $f\left(x^{\prime}\right), f\left(x^{\prime \prime}\right) \in Y_{n}^{i_{n}}$. Our assumptions on the $Y_{j}^{i_{j}}$ imply that $c^{\prime}$ is between $f\left(x^{\prime}\right)$ and $f\left(x^{\prime \prime}\right)$ (in $\left.Y_{n}^{i_{n}}\right)$. By monotonicity and continuity of $f$ we can find some $d=\left(d_{1}, \ldots, d_{n-1}\right) \in I_{1} \times \cdots \times I_{n-1}$ such that $f(d)=c^{\prime}$ and $d_{j}$ is between $x_{j}^{\prime}$ and $x_{j}^{\prime \prime}$ in $I_{j}$ for each $j$. Note that $E\left(x_{j}^{\prime}, x_{j}^{\prime \prime}\right)$ for $j=1, \ldots, n-1$, so our assumptions (convexity, and convexity of $E$-classes) on the $Y_{j}^{i_{j}}$ for $j=1, \ldots, n-1$ yield $\pi^{n-1}(d)=x$. We have found $c^{\prime} \in I_{n}$ such that $\pi^{n-1}\left(\left\{z \in C: f(z)=c^{\prime}\right\}\right)$ contains $U$ (an open definable set in $\pi\left(I_{1}\right) \times \cdots \times \pi\left(I_{n-1}\right)$ ). The induction hypothesis implies that $\left\{z \in C: f(z)=c^{\prime}\right\}$ has dimension $n-1$, so contains an open definable (in $\bar{M}$ ) subset of $C$. This gives a contradiction with the fact that we have infinitely many such $c^{\prime}$. The claim is proved.

We now return to our definable subset $Y$ of $G$ such that $Y=\left\{x_{1}+\cdots+x_{n}\right.$ : $\left.\left(x_{1}, \ldots, x_{n}\right) \in Z\right\}$. As $\pi$ is a homomorphism, $G / G^{00} \supset \pi(Y)=\left\{\pi\left(x_{1}\right)+\cdots+\pi\left(x_{n}\right)\right.$ : $\left.\left(x_{1}, \ldots, x_{n}\right) \in Z\right\}=\left\{y_{1}+\cdots+y_{n}:\left(y_{1}, \ldots, y_{n}\right) \in \pi_{n}(Z)\right\}$. By the Claim, $\pi_{n}(Z)$ has dimension $<n$, hence $\pi(Y)$ does too. This completes the proof of Lemma 8.8.

The final point is easy:

Lemma 8.9. Let $Z \subset J$ be definable in $\bar{M}^{*}$ of (semi-o-minimal) dimension $<n$, and Haar measurable in $G / G^{00}$. Then $Z$ has Haar measure 0 .

Proof. The proof will be by induction on $\operatorname{dim}(Z)=k$. For $k=0, Z$ is finite so it is clear. Now let $Z$ be of dimension $k>0$.

Claim. For any $r$ there are $a_{1}, \ldots, a_{r} \in J$ such that $\operatorname{dim}\left(a_{i} Z \cap a_{j} Z\right)<k$ for all $i \neq$ $j \leq r$.

Proof of Claim. Work in a saturated elementary extension $N$ of $\bar{M}^{*}$. First note that if $a \in J(N)$ is generic (in the $o$-minimal sense) over the base model (so $\operatorname{dim}\left(a / \bar{M}^{*}\right)=n$ ) then $\operatorname{dim}(Z \cap a Z)<k$. For if not choose $c \in Z \cap a Z$ with $\operatorname{dim}\left(c / \bar{M}^{*}, a\right)=k$. As $c \in Z$, $\operatorname{dim}\left(c / \bar{M}^{*}\right) \leq k$ so equals $k$ and $c$ is independent of $a$ over $\bar{M}^{*}$ in the $o$-minimal sense. So $\operatorname{dim}\left(a^{-1}, c / \bar{M}^{*}\right)=n+k$. A dimension calculation implies that $\operatorname{dim}\left(c \cdot a^{-1} / \bar{M}^{*}\right)=n$. But $c \cdot a^{-1} \in Z$ and $\operatorname{dim}(Z)<n$, a contradiction.

Now let $a_{1}, \ldots, a_{r} \in J(N)$ be generic independent (in the $o$-minimal sense) over the base model, namely $\operatorname{dim}\left(a_{1}, \ldots, a_{r} / \bar{M}^{*}\right)=n r$. Then one sees from the above that $\operatorname{dim}\left(a_{i} Z(N) \cap a_{j} Z(N)\right)<k$ for $i \neq j$. By definability of dimension we can find such $a_{1}, \ldots, a_{r}$ in $J$. This proves the claim. 
Note that as $Z$ is measurable in $G / G^{00}$, so is any intersection of translates of $Z$. Hence by the induction hypothesis and the claim, each $a_{i} Z \cap a_{j} Z(i \neq j)$ has Haar measure 0 . But then the measure of the union of the $a_{i} Z$ is $r$ times the measure of $Z$. So (by choosing $r$ large) this forces $Z$ to have measure 0 . The proof is complete.

Proof of Theorem 8.1. If $Y \subset G$ is definable in $\bar{M}$ with dimension $<n$, then $\pi(Y)$ is closed, so measurable in $G / G^{00}$; but by Lemma $8.8, \pi(Y)$ has dimension $<n$ as a definable subset of $J$, hence has Haar measure 0 in $G / G^{00}$ by Lemma 8.9. By 10.5 in [16] we obtain compact domination of $G$ by $G / G^{00}$ (equipped with its Haar measure).

We conclude the body of the paper by proving that the topologies on $J$ and $G / G^{00}$ coincide. Our proof will make use of a little more "theory" some of which is of interest in its own right.

We begin with an arbitrary complete theory $T$ with $N I P$. For $\phi(x, y) \in L$ by a (complete) global $\phi$-type we mean a maximal consistent collection of formulas of the form $\phi(x, c), \neg \phi(x, c)$ for $c \in \bar{M}$.

Lemma 8.10. Let $M_{0}$ be a small model, $\phi(x, y) \in L$ and $p_{0}(x)$ a complete global $\phi$-type which is $\operatorname{Aut}\left(\bar{M} / M_{0}\right)$-invariant. Then $p_{0}$ extends to a complete global type $p(x)$ which is $\operatorname{Aut}\left(\bar{M} / M_{1}\right)$-invariant for some small model $M_{1}$ (i.e. in earlier terminology $p$ is invariant).

Proof. We will give two proofs. The first, our original one, starts in analogy with Step I of Lemma 5.8. That is, we find a small model $M_{1}$ containing $M_{0}$ and a Keisler measure $\mu_{x}$ over $M_{1}$ which has a unique extension to a global Keisler measure $\mu^{\prime}$ which extends $p_{0}$. (Again to carry out the required construction, we need to know that if $\mu_{i}$ are Keisler measures over models $M_{i}$ for $i<\omega$ such that for $i<j, M_{i} \subset M_{j}, \mu_{i} \subset \mu_{j}$, and each $\mu_{i}$ has an extension to a global Keisler measure extending $p_{0}$, then $\bigcup_{i} \mu_{i}$ has an extension to a global Keisler measure extending $p_{0}$. This follows from compactness of the space of global Keisler measures.) Note that as $p_{0}$ is $\operatorname{Aut}\left(\bar{M} / M_{1}\right)$-invariant, so is $\mu^{\prime}$. By the proof of 4.5, we obtain some global type $p^{\prime}(x)$ which is $M_{1}$-invariant and extends $p_{0}$.

The second proof is direct (showing in addition that we can choose $M_{1}$ to be $M_{0}$ ) and uses a result by Chernikov and Kaplan [6] which was proved subsequent to the first draft (2007) of this paper. The result is that in an NIP context, if $M$ is a model, and $\phi(x, b)$ any formula, then $\phi(x, b)$ divides over $M$ if and only if $\phi(x, b)$ forks over $M$. So view $p_{0}$ as a partial type over $\bar{M}$. As $p_{0}$ is $\operatorname{Aut}\left(\bar{M} / M_{0}\right)$-invariant, clearly no formula in $p_{0}$ divides over $M_{0}$. By the result just alluded to, no formula in $p_{0}(x)$ forks over $M_{0}$, hence (by compactness) $p_{0}$ extends to a global complete type $p(x)$ which does not fork over $M_{0}$. By Proposition 2.1(ii), $p(x)$ is $\operatorname{Aut}\left(\bar{M} / M_{0}\right)$-invariant.

Corollary 8.11 (Strong Borel definability for invariant $\phi$-types). Let $p_{0}$ be a complete global $\phi$-type which is $M_{0}$-invariant. Then $X=\left\{b: \phi(x, b) \in p_{0}(x)\right\}$ is a finite Boolean combination of type-definable (over $M_{0}$ ) sets.

Proof. By Lemma 8.10 and Proposition 2.6, $X$ is a finite Boolean combination of typedefinable (over $M_{1}$ ) sets $Y_{i}$ for some small model $M_{1}$ containing $M_{0}$. Let $Y_{i}^{\prime}$ be $\{b$ : 
$\left.\exists c \in Y_{i}, \operatorname{tp}\left(c / M_{0}\right)=\operatorname{tp}\left(b / M_{0}\right)\right\}$. Then $Y_{i}^{\prime}$ is type-definable and $M_{0}$-invariant, hence type-definable over $M_{0}$. And $X$ is the same finite Boolean combination of the $Y_{i}^{\prime}$.

Here is the application which will be relevant to our concerns:

Lemma 8.12. Let $\bar{M}$ be a saturated model, and $M_{0}$ a small submodel. Let $\bar{M}^{*}$ be the Shelah expansion discussed earlier. Suppose that $X$ is definable in $\bar{M}^{*}$ and is $\operatorname{Aut}\left(\bar{M} / M_{0}\right)$ invariant. Then $X$ is a finite Boolean combination of type-definable (over $M_{0}$ in the structure $\bar{M}$ ) sets.

Proof. As $\operatorname{Th}\left(\bar{M}^{*}\right)$ has quantifier elimination, there is some complete type $p(x)$ over $\bar{M}$ and $L$-formula $\phi(x, y)$ such that $b \in X$ iff $\phi(x, b) \in p$ (for all $b \in \bar{M}$ ). Let $p_{0}=p \mid \phi$. So $p_{0}$ is a complete global $M_{0}$-invariant $\phi$-type and we can apply Corollary 8.11.

We now return to the setting and notation of Theorem 8.1 and its proof. In particular $J$ is the set $G / G^{00}$ viewed as a definable (compact Lie) group in $\bar{M}^{*}$ and we just say $G / G^{00}$ for $G / G^{00}$ with the logic topology, another compact Lie group. $M_{0}$ is a fixed small submodel of $\bar{M}$.

Lemma 8.13. (i) Suppose $Z$ is a definable (in $\bar{M}^{*}$ ) subset of $J$. Then $Z$ is measurable in $G / G^{00}$.

(ii) If moreover $H$ is a definable (in $\bar{M}^{*}$ ) subgroup of $J$ then $H$ is closed in $G / G^{00}$.

Proof. (i) Clearly $\pi^{-1}(Z)$ is a definable set in $\bar{M}^{*}$ and whether or not $x \in \pi^{-1}(Z)$ depends on $\operatorname{tp}\left(x / M_{0}\right)$ in $\bar{M}$. Hence $\pi^{-1}(Z)$ satisfies the assumptions of Lemma 8.12 so is a finite Boolean combination of type-definable sets. Note that $\pi^{-1}(Z)$ is $G^{00}$-invariant. It follows that in fact $\pi^{-1}(Z)$ is a finite Boolean combination of type-definable sets each of which is $G^{00}$-invariant. (Justification: By 8.12 and the proof of $8.11, \pi^{-1}(Z)$ is a finite disjoint union of sets $X_{i} \backslash Y_{i}$, where $X_{i}, Y_{i}$ are type-definable and without loss $Y_{i} \subseteq X_{i}$. Let us consider for simplicity the case where $i=2$. Let $W_{i}=X_{i} \backslash Y_{i}$ for $i=1,2$. Then $W_{1} \cup W_{2}$ is $G^{00}$-invariant, namely a union of translates of $G^{00}$. Let us write $[X]$ for the the smallest $G^{00}$-invariant set including $X$, which note will be type-definable if $X$ is. Then we leave it to the reader to check that

$$
W_{1} \cup W_{2}=\left(\left(\left[X_{1}\right] \backslash\left[Y_{1}\right]\right) \cup\left(\left[X_{2}\right] \backslash\left[Y_{2}\right]\right) \cup\left[Y_{1} \cap X_{2}\right] \cup\left[X_{1} \cap Y_{2}\right]\right) \backslash\left[Y_{1} \cap Y_{2}\right],
$$

a finite Boolean combination of type-definable $G^{00}$-invariant sets, as required.) It now follows that $Z=\pi\left(\pi^{-1}(Z)\right)$ is a finite Boolean combination of closed subsets of $G / G^{00}$, so Borel and measurable.

(ii) As $G / G^{00}$ is separable, any finite Boolean combination of closed sets is a countable intersection of opens, that is, a $G_{\delta}$-set. So applying the conclusion of the proof of (i) to $H$ we see that $H$ is a $G_{\delta}$-set in $G / G^{00}$. Now the closure $\bar{H}$ of $H$ is a subgroup of $G / G^{00}$. Moreover $H$ and thus each of its translates in $\bar{H}$ is dense in $\bar{H}$. But any two dense $G_{\delta}$ 's must intersect. Hence $H=\bar{H}$.

Remark 8.14. Again the above lemma holds at various levels of generality: (i) holds assuming just $T$ has NIP and (ii) holds if in addition $G / G^{00}$ is separable. Note that (ii) 
is saying that any subgroup of $G$ which contains $G^{00}$ and is definable in $\bar{M}^{*}$ is typedefinable in $\bar{M}$. In particular the groups $\operatorname{Stab}_{\psi}(p)$ from the proof of Lemma 8.2 are typedefinable, so using the DCC we obtain another proof that $G^{00}$ is a finite intersection of the $\operatorname{Stab}_{\psi}(p)$ 's.

Proposition 8.15. The topologies on $J$ and $G / G^{00}$ coincide.

Proof. As the group structures on $J$ and $G / G^{00}$ coincide, and both $J$ and $G / G^{00}$ are compact (Hausdorff) groups, it suffices to show that any open neighbourhood $U$ of the identity $e$, in the sense of $J$, contains a neighbourhood of $e$ in the sense of $G / G^{00}$. Let $h$ denote the Haar measure on $G / G^{00}$. Let $W$ be a definable neighbourhood of $e$ in $J$ such that $W^{-1} \cdot W \cdot W^{-1} \cdot W$ is contained in $U$. Then $W$ is clearly generic in $J$ (finitely many translates of $W$ cover $J$ ) by compactness of $J$ for example. By Lemma 8.13, $W$ is measurable in $G / G^{00}$, so $h(W)>0$. As pointed out in the proof of the Claim in Section 6 of [16] it follows that $W^{-1} \cdot W$ has interior in $G / G^{00}$. (A direct proof: By regularity of the Haar measure $h, W$ contains a closed set of positive measure, so we may assume that $W$ is closed. Hence $Z=W^{-1} \cdot W$ is also closed. If by way of contradiction $Z$ has no interior then the same holds for any finite union of translates of $Z$. So we find $a_{1}, a_{2}, \ldots$ in $G / G^{00}$ such that the $a_{i} W$ are disjoint, contradicting $h(W)>0$.) So $W^{-1} \cdot W \cdot W^{-1} \cdot W$ contains a neighbourhood of $e$ (in $G / G^{00}$ ) as required.

It would be interesting to give a more explicit proof of Proposition 8.15. For example if $\operatorname{dim}(G)=1$ then the analysis in the proof of Proposition 3.5 of [33] implies directly (in hindsight) that the logic topology equals the $o$-minimal topology on $G / G^{00}$ and coincides with $S^{1}$.

Note that it follows from 8.9 and 8.13 that for any definable (in $\bar{M}^{*}$ ) subset $Z$ of $G / G^{00}, Z$ has Haar measure 0 iff it has dimension $<\operatorname{dim}\left(G / G^{00}\right)$. In any case the proof of Theorem 8.1 together with Proposition 8.15 says that $G$ is $o$-minimally compactly dominated, i.e.

(*) Let $\bar{M}^{\prime}$ be the expansion of $\bar{M}$ obtained by adding a predicate for $G^{00}$. Then $G / G^{00}$ is a semi-o-minimal compact Lie group in $\bar{M}^{\prime}$ with topology coinciding with its topology as a bounded hyperdefinable group in $\bar{M}$, AND for any definable (in $\bar{M}$ ) subset $X$ of $G$, the set of $b \in G / G^{00}$ such that $\pi^{-1}(b)$ meets both $X$ and its complement (which is of course definable in $\bar{M}^{\prime}$ ) has dimension $<\operatorname{dim}\left(G / G^{00}\right)$, EQUIVALENTLY, has Haar measure 0 .

Let us first remark that $(*)$ also holds for $X$ definable in $\bar{M}^{*}$. This is because $X=Y \cap$ $G(\bar{M})$ for some subset $Y$ of $G(N)$ definable (with parameters) in a saturated elementary extension $N$ of $\bar{M}$. As $\left(G / G^{00}\right)(N)=\left(G / G^{00}\right)(\bar{M})$ and $(*)$ is valid working in $N$, we deduce that the set of $b \in G / G^{00}$ such that $\pi^{-1}(b)$ meets both $X$ and its complement (in $G(M)$ ), which again note is a subset of $G / G^{00}$ definable in $\bar{M}^{*}$, has Haar measure 0 . We conclude:

Corollary 8.16. Let $G$ be a commutative group which is definably compact and definable in the saturated o-minimal expansion $\bar{M}$ of a real closed field. Let $\bar{M}^{*}$ be the Shelah expansion of $\bar{M}$. Let $J=G / G^{00}$ as a definable group in $\bar{M}^{*}$. Then $G$ is dominated by $J$ 
in the weakly o-minimal theory $\operatorname{Th}\left(\bar{M}^{*}\right)$ : namely, working even in a saturated elementary extension of $\bar{M}^{*}$, if $X$ is a definable subset of $G$ then the set of $b \in J$ such that $\pi^{-1}(b)$ meets both $X$ and its complement, has (o-minimal) dimension $<\operatorname{dim}(J)$.

One can ask what the formal content and implications of the compact domination statement $(*)$ are (either in general, or restricted to the $o$-minimal context). For example, from [16] we know that if a definable group is compactly dominated then it has the $f_{s g}$ property and a unique invariant Keisler measure. Of course the proof of Theorem 8.1 (or statement $(*)$ ) depends on $G$ having the $f s g$ property as well as the knowledge of torsion points from [8] (for definably compact $G$ ). It would be interesting to try to recover the torsion points statement directly from compact domination. Namely

Question. (o-minimal context.) Suppose the commutative definably connected definable group $G$ is $o$-minimally compactly dominated, i.e. statement $(*)$ holds. Can one prove directly that $\operatorname{dim}(G)=\operatorname{dim}\left(G / G^{00}\right)$ (and so conclude using the torsion-freeness and divisibility of $G^{00}$ that for each $p$ the $p$-torsion of $G$ is $\left.(\mathbb{Z} / p \mathbb{Z})^{\operatorname{dim}(G)}\right)$ ?

The divisibility of $G^{00}$ was proved in [4], and the proof of its torsion-freeness is contained in the proof of Lemma 8.2 in [16] which does not make use of knowledge of torsion points.

A final question is whether given a definable group $G$ in a NIP theory $T$, there are some reasonable assumptions which imply that $G / G^{00}$ is semi-o-minimal (in the Shelah expansion) or at least $o$-minimally analysable. A possible assumption would be that $G$ has the DCC on type-definable subgroups of bounded index (equivalently, $G / G^{00}$ with its logic topology is a compact Lie group).

\section{A. Appendix: On Skolem functions for $o$-minimal definable sets}

We prove a general result, Proposition A.2 below, and show that it applies to the situation in Section 8 to yield Proposition 8.6(iii).

Let us fix a saturated structure $N$, and a $\emptyset$-definable set $X$ in $N$ such that for some $\emptyset$-definable dense linear ordering without endpoints $<$ on $X, X$ is $o$-minimal in $N$ with respect to $<$. We will freely adapt $o$-minimality results for the absolute case (where $X$ is the universe of $N$ ) to this relative case.

By $X^{\mathrm{eq}}$ we mean $N^{\mathrm{eq}} \cap \operatorname{dcl}(X)$. It is known that the $o$-minimal dimension theory on $X$ extends smoothly to $X^{\text {eq }}$ (see for example Section 3.1 of [28]). Namely for any set $A$ of parameters from $N$, and $c \in X^{\text {eq }}$, we have a natural number $\operatorname{dim}(c / A)$ such that $\operatorname{dim}$ is subadditive $(\operatorname{dim}((c, d) / A)=\operatorname{dim}(c / d A)+\operatorname{dim}(d / A)), \operatorname{dim}(c / A)=0$ iff $c \in \operatorname{acl}(A)$, and for $c$ an element (rather than tuple) of $X, \operatorname{dim}(c / A)=1$ if $c \notin \operatorname{acl}(A)$.

Definition A.1. We say that $X$ is untrivial if whenever $\left(a_{i}: i<\omega\right)$ is a sequence of elements of $X$ such that $a_{i} \notin \operatorname{acl}\left(a_{0}, \ldots, a_{i-1}\right)$ for all $i$ then there is some $n$ and $b \in X^{\text {eq }}$ such that $b \in \operatorname{acl}\left(a_{0}, \ldots, a_{n}\right) \backslash \operatorname{acl}\left(a_{0}, \ldots, a_{n-1}\right)$ and $a_{n} \notin \operatorname{acl}(b)$.

The main result here is: 
Proposition A.2. Suppose $X$ is untrivial. Then for any elementary substructure $N_{0}$ of $N$, $X$ has definable Skolem functions in $N$ over $N_{0}$.

Let us note for the record that if $X$ is untrivial then $X$ is also untrivial over any small set $B$ of parameters from $N$. For if $a_{i} \notin \operatorname{acl}\left(a_{0}, \ldots, a_{i-1}, B\right)$ for $i=0, \ldots, n$ say, then $\left(a_{0}, \ldots, a_{n}\right)$ is independent of $B$ in the $o$-minimal sense, that is, $\operatorname{dim}\left(a_{0}, \ldots, a_{n} / \emptyset\right)=$ $\operatorname{dim}\left(a_{0}, \ldots, a_{n} / B\right)=n$, and moreover for any $b \in X^{\mathrm{eq}}$ with $b \in \operatorname{acl}\left(a_{0}, \ldots, a_{n}\right)$, $\left(a_{0}, \ldots, a_{n}, b\right)$ is also independent of $B$ over $\emptyset$, in this dimension sense. So if in addition $b \notin \operatorname{acl}\left(a_{0}, \ldots, a_{n-1}\right)$ and $a_{n} \notin \operatorname{acl}(b)$ then this will also hold over $B$. This will be used in the proofs below. On the other hand, in the application of Proposition A.2 below (proof of 8.6(iii)), our discussion gives directly untriviality over any set of parameters of the relevant $o$-minimal set. So there would be no harm adding this hypothesis to A. 2 for the skeptical reader.

Before entering the proof of Proposition A.2 we give a lemma which makes use of the fundamental work of Peterzil and Starchenko [29].

Lemma A.3. Suppose $X$ is untrivial. Then for any $a \in X$ with $a \notin \operatorname{acl}(\emptyset)$ there is some interval $(c, d)$ containg a such that the definable set $(c, d)$ has definable Skolem functions in $N$ after naming some parameters from $X$.

Proof. Let $a_{i}$ for $i<\omega$ be realizations of $\operatorname{tp}(a)$ such that $a_{i+1} \notin \operatorname{acl}\left(a_{0}, \ldots, a_{i}\right)$ for all $i$. Let $n$ and $b$ be given by untriviality of $X$. Then a dimension (or independence) calculation shows that $a_{n} \in \operatorname{acl}\left(a_{0}, \ldots, a_{n-1}, b\right)$. We also have $a_{n} \notin \operatorname{acl}\left(a_{0}, \ldots, a_{n-1}\right)$ and $a_{n} \notin$ $\operatorname{acl}(b)$. Let $\bar{b}$ be a finite tuple of elements of $X$ such that $b \in \operatorname{dcl}(\bar{b})$ and $\bar{b}$ is independent of $a_{n}$ over $b$ (i.e. $\left.\operatorname{dim}\left(\bar{b} / b a_{n}\right)=\operatorname{dim}(\bar{b} / b)\right)$. So we have $a_{n} \in \operatorname{acl}\left(a_{0}, \ldots, a_{n-1}, \bar{b}\right)$ but $a_{n} \notin \operatorname{acl}\left(a_{0}, \ldots, a_{n-1}\right)$ and $a_{n} \notin \operatorname{acl}(\bar{b})$. We may assume $a=a_{n}$. By Claim 1.25 of [27], $a$ is "PS-nontrivial", that is, there is some infinite open interval $I$ containing $a_{n}$ and a definable continuous function $F: I \times I \rightarrow X$, strictly monotone in each argument. By Theorem 1.1 of [29] there is a convex type-definable ordered divisible abelian group $H \subset X$, containing $a$ (where the ordering is the restriction of $<$ to $H$ ). Let $c, d \in H$ be such that $c<a<d$, and let $A$ be some set of parameters from $X$ containing $a, c, d$ and over which the group operation on $H$ is type-definable. Then it is clear that $(c, d)$ has definable Skolem functions in $N$ over $A$.

Proof of Proposition A.2. We begin with some reductions. Let $N_{0}$ be a (small) definably closed substructure of $N$ (or even $N^{\mathrm{eq}}$ ). We will say that $N_{0}$ satisfies Tarski-Vaught with respect to $X$ if any formula over $N_{0}$ which is satisfied in $N$ by some tuple from $X$ is satisfied by a tuple from $N_{0} \cap X$. It is clearly enough to restrict ourselves to formulas $\phi(x)$ over $N_{0}$ where $x$ is a single variable ranging over $X$. In any case clearly any elementary substructure of $N$ satisfies Tarski-Vaught with respect to $X$. So by using compactness, in order to prove A. 2 it is enough to prove:

(*) whenever $N_{0}$ satisfies Tarski-Vaught with respect to $X$, then for any tuple $\bar{c}$ from $N$, $\operatorname{dcl}\left(N_{0}, \bar{c}\right)$ satisfies Tarski-Vaught with respect to $X$.

If in the context of $(*), \phi(x)$ is a formula over $N_{0} \bar{c}$ with $x$ ranging over elements of $X$, then $\phi(x)$ defines a finite union $X_{0}$ of intervals and points from $X$. The boundary points 
of $X_{0}$ are in $\operatorname{dcl}\left(M_{0}, \bar{c}\right)$, and $X_{0}$ is defined over the set of these boundary points. So in order to prove $(*)$ it suffices to prove:

(**) whenever $N_{0}$ satisfies Tarski-Vaught with respect to $X$, and $\bar{c}$ is a finite tuple of elements of $X$, then $\operatorname{dcl}\left(N_{0}, \bar{c}\right)$ satisfies Tarski-Vaught with respect to $X$.

We can of course prove $(* *)$ by adding one element from $\bar{c}$ at a time. Hence it suffices to prove:

(***) whenever $N_{0}$ satisfies Tarski-Vaught in $N$ with respect to $X$, and $a$ is an element of $X$, then $\operatorname{dcl}\left(N_{0}, a\right)$ satisfies Tarski-Vaught in $N$ with respect to $X$.

The rest of the proof is devoted to proving $(* * *)$. We will suppose that $(* * *)$ fails and aim for a contradiction. Using $o$-minimality of $X$, the failure of $(* * *)$ is equivalent to the existence of an element $a \in X$, and $d \in\left(\operatorname{dcl}\left(N_{0}, a\right) \cap X\right) \cup\{+\infty,-\infty\}$ such that the formula $a<x<d$ (if $d>a$ ) or $d<x<a$ (if $d<a$ ) isolates a complete type over $N_{0} a$. There is no harm in assuming that $d>a$.

Let $p=\operatorname{tp}\left(a / N_{0}\right)$ and $P$ be the set of realizations of $p$ in $N$. Then we can write $d=g(a)$ for some $N_{0}$-definable (possibly constant) function $g$ on $P$. There are two cases depending on whether or not $g(a) \in N_{0}$.

Case (i): $g(a) \notin N_{0} \cup\{+\infty\}$. Note that $g(a)$ realizes $p$ too (as $N_{0} \cap[a, g(a)]=\emptyset$ ) and that $g$ is an $N_{0}$-definable strictly increasing function from $P$ onto itself.

Claim I. There is no $\bar{e}$ from $X$ and $N_{0} \bar{e}$-definable function $f_{\bar{e}}$ such that for all $a^{\prime} \in P$, $a^{\prime}<f_{\bar{e}}\left(a^{\prime}\right)<g\left(a^{\prime}\right)$.

Proof of Claim I. Otherwise by compactness there is $\theta(x) \in p$ such that

$$
N \models \forall x\left(\theta(x) \rightarrow\left(x<f_{\bar{e}}(x)<g(x)\right)\right) .
$$

As $N_{0}$ satisfies Tarski-Vaught in $N$ with respect to $X$, there is $\bar{e}^{\prime}$ from $N_{0}$ such that

$$
N \models \forall x\left(\theta(x) \rightarrow x<f_{\bar{e}^{\prime}}(x)<g(x)\right) .
$$

But then $a<f_{\bar{e}^{\prime}}(a)<g(a)$, contradicting the fact that $a<x<g(a)$ isolated a complete type over $N_{0} a$. Claim I is proved.

Claim II. The interval $(a, g(a))$ has definable Skolem functions in $N$ over some set of parameters from $X$.

Proof of Claim II. As $p$ is a complete nonalgebraic 1-type of $X$ over $N_{0}$, it follows from Lemma A.3 that for some $c$ with $a<c<g(a),(a, c)$ has definable Skolem functions in $N$ after naming some parameters from $X$. It follows that for any $a^{\prime}$ realizing $p$ and $c^{\prime} \in\left(a^{\prime}, g\left(a^{\prime}\right)\right),\left(a^{\prime}, c^{\prime}\right)$ has definable Skolem functions in $N$ over parameters from $X$. Now (with $c \in(a, g(a))), a<c<g(a)<g(c)$. So both $(a, c)$ and $(c, g(a))$ have definable Skolem functions (after naming parameters). Hence so does $(a, g(a))$.

Claim III. There are realizations $a_{i}$ of $p$ for $i<\omega$ such that, writing $I_{k}$ for the interval $\left(a_{k}, g\left(a_{k}\right)\right)$, we have for all $k, \operatorname{dcl}_{N_{0}}\left(I_{0} \cup \cdots \cup I_{k-1} \cup\left\{a_{0}, a_{1}, \ldots, a_{k-1}, a_{k}\right\}\right) \cap I_{k}=\emptyset$. (Here $\mathrm{dcl}_{N_{0}}(A)$ denotes definable closure in $N$ of $A \cup N_{0}$.) 
Proof of Claim III. Suppose we have already constructed $a_{0}, \ldots, a_{k-1}$, and suppose for a contradiction that

(甘) for all $a^{\prime} \in P, \operatorname{dcl}_{N_{0}}\left(I_{0} \cup \cdots \cup I_{k-1} \cup\left\{a_{0}, \ldots, a_{k-1}, a\right\}\right) \cap(a, g(a)) \neq \emptyset$.

By Claim II, let $\bar{e}$ be a tuple from $X$ such that each of $I_{0}, \ldots, I_{k-1}$ has definable Skolem functions in $N$ over $\bar{e}$ (so also over $N_{0} \bar{e}$ ), and we may assume that $a_{0}, \ldots, a_{k-1}$ are in $\bar{e}$. Now fix $a^{\prime} \in P$. By ( $\left.\sharp\right)$ there is an $N_{0}$-definable function $f\left(w, z_{0}, \ldots, z_{k-1}, x\right)$ such that there are tuples $c_{0}, \ldots, c_{k-1}$ from $I_{0}, \ldots, I_{k-1}$ respectively with $f\left(\bar{e}, c_{0}, \ldots, c_{k-1}, a^{\prime}\right)$ $\in\left(a^{\prime}, g\left(a^{\prime}\right)\right)$. Hence there are such $c_{0}, \ldots, c_{k-1}$ which are in addition contained in $\operatorname{dcl}_{N_{0}}\left(\bar{e}, a^{\prime}\right)$. So we have shown that for every $a^{\prime} \in P, \operatorname{dcl}_{N_{0}}\left(\bar{e}, a^{\prime}\right) \cap\left(a^{\prime}, g\left(a^{\prime}\right)\right) \neq \emptyset$. By compactness there is an $N_{0} \bar{e}$-definable function $f_{\bar{e}}(-)$ such that for all $a^{\prime} \in P$, $f_{\bar{e}}\left(a^{\prime}\right) \in\left(a^{\prime}, g\left(a^{\prime}\right)\right)$. This contradicts Claim I. Claim III is proved.

Note that the construction in Claim III goes through with the additional constraint that $a_{k} \notin \operatorname{acl}_{N_{0}}\left(a_{0}, \ldots, a_{k-1}\right)$. So we can apply the untriviality of $X$ (over any set of parameters) to find $n$ and $b \in X^{\mathrm{eq}}$ such that $b \in \operatorname{acl}_{N_{0}}\left(a_{0}, \ldots, a_{n}\right) \backslash \operatorname{acl}_{N_{0}}\left(a_{0}, \ldots, a_{n-1}\right)$ (whence $\left.a_{n} \in \operatorname{acl}_{N_{0}}\left(a_{0}, \ldots, a_{n-1}, b\right)\right)$, and $a_{n} \notin \operatorname{acl}_{N_{0}}(b)$. This leads quickly to a contradiction as we now show. At this point we will work over $N_{0}$ for notational simplicity.

First choose $c_{i}, d_{i}$ for $i<n$ such that $g^{-1}\left(a_{i}\right)<c_{i}<a_{i}<d_{i}<g\left(a_{i}\right)$, and $\left(c_{0}, d_{0}, \ldots, c_{n-1}, d_{n-1}\right)$ is independent of $\left(a_{n}, b\right)$ (in the $o$-minimal sense). Then since $a_{n} \notin \operatorname{acl}(b)$, we also have $a_{n} \notin \operatorname{acl}\left(c_{0}, d_{0}, \ldots, c_{n-1}, d_{n-1}, b\right)$. On the other hand, as $a_{n} \in \operatorname{dcl}\left(a_{0}, \ldots, a_{n-1}, b\right)$ there is a $\emptyset$-definable function $f$ such that

$$
\vDash \exists x_{0}, \ldots, x_{n-1}\left(\left(\bigwedge_{i<n} c_{i}<x_{i}<d_{i}\right) \wedge f\left(b, x_{0}, \ldots, x_{n-1}\right)=a_{n}\right) .
$$

As $\operatorname{tp}\left(a_{n} / c_{0}, d_{0}, \ldots, c_{n-1}, d_{n-1}, b\right)$ is not algebraic, its set of realizations contains an open interval around $a_{n}$. Hence we can find some $b_{n} \in I_{n}$ and $b_{0}, \ldots, b_{n-1} \in X$ such that for each $i<n, c_{i}<b_{i}<d_{i}$ and $f\left(b_{0}, \ldots, b_{n-1}, b\right)=b_{n}$. Now put $b_{i}^{\prime}=b_{i}$ if $b_{i} \geq a_{i}$, and $b_{i}^{\prime}=g\left(b_{i}\right)$ if $b_{i}<a_{i}$. So $b_{i}^{\prime} \in I_{i} \cup\left\{a_{i}\right\}$ for each $i$. As $b \in \operatorname{acl}\left(a_{0}, \ldots, a_{n}\right)$ we conclude that $b_{n} \in \operatorname{dcl}\left(b_{0}^{\prime}, \ldots, b_{n-1}^{\prime}, a_{0}, \ldots, a_{n-1}, a_{n}\right)$. As $b_{n} \in I_{n}$ this contradicts the construction of the $a_{i}$. This contradiction completes the proof under Case (i).

Case (ii): $g(a) \in N_{0} \cup\{+\infty\}$. So $g(a)$ is either a point of $X$ in $N_{0}$, or $+\infty$. Let $d=$ $\left.g(a) \in N_{0} \cup\{\infty\}\right)$. So clearly $p(x)=\operatorname{tp}\left(a / N_{0}\right)$ is the complete type over $N_{0}$ saying that $x \in X, x<d$ and $x>c$ for all $c \in X\left(N_{0}\right)$ such that $c<d$.

Claim IV. $P$ (the set of realizations of $p$ ) is indiscernible, as an ordered set, over $N_{0}$. Namely for each $n, p\left(x_{1}\right) \cup \cdots \cup p\left(x_{n}\right) \cup\left\{x_{1}<\cdots<x_{n}\right\}$ extends to a unique complete type over $N_{0}$.

Proof of Claim IV. By induction. The case $n=2$ is given to us, as $a<x<d$ isolates a complete 1-type over $N_{0} a$. Now assume the conclusion is true for $n \geq 2$, and prove it for $n+1$. Let $a_{1}<\cdots<a_{n}$ realize $p$. It suffices to show that $a_{n}<x<d$ isolates a complete 1-type over $N_{0} \cup\left\{a_{1}, \ldots, a_{n}\right\}$, and for that it is enough to prove that dcl $\left(N_{0}, a_{1}, \ldots, a_{n}\right) \cap$ $\left(a_{n}, d\right)=\emptyset$. If not, $a_{n}<f\left(a_{1}, \ldots, a_{n}\right)<d$ for some $N_{0}$-definable function $f$. But by induction hypothesis, $\operatorname{tp}\left(a_{n} / N_{0} a_{1} \ldots a_{n-1}\right)$ is isolated by $a_{n-1}<x<d$. Hence $N \models$ 
$(\forall x)\left(\left(a_{n-1}<x<d\right) \rightarrow\left(x<f\left(a_{1}, \ldots, a_{n-1}, x\right)<d\right)\right)$. Now we use the hypothesis that $N_{0}$ satisfies Tarski-Vaught in $N$ with respect to $X$ to find $a_{1}^{\prime}<\cdots<a_{n-1}^{\prime}$ in $X\left(N_{0}\right)$, all less than $d$, such that $x<f\left(a_{1}^{\prime}, \ldots, a_{n-1}^{\prime}, x\right)<d$ for any $x$ with $a_{n-1}^{\prime}<x<d$. But our realization $a$ of $p$ is such an $x$, and we get a contradiction with the fact that the formula $a<x<d$ isolates a complete type over $N_{0} a$. This finishes the proof of Claim IV.

Note that by Claim (IV) any set of distinct realizations of $p$ is algebraically independent over $N_{0}$. This is clearly incompatible with the untriviality of $X$ over $N_{0}$ : Let $a_{0}, \ldots, a_{n}, b$ witness untriviality (over $N_{0}$ ) where $a_{0}<\cdots<a_{n}$ are realizations of $p$. As $a_{n} \notin \operatorname{acl}\left(b, N_{0}\right)$ there is $a_{n}^{\prime}$ realizing $\operatorname{tp}\left(a_{n} / N_{0}, b\right)$ and different from each of $a_{0}, \ldots, a_{n}$. Let $a_{0}^{\prime}, \ldots, a_{n-1}^{\prime}$ be such that $\operatorname{tp}\left(a_{0}, \ldots, a_{n} / N_{0} b\right)=\operatorname{tp}\left(a_{0}^{\prime}, \ldots, a_{n}^{\prime} / N_{0} b\right)$. Then $a_{n}^{\prime} \in$ $\operatorname{acl}\left(N_{0}, a_{0}^{\prime}, \ldots, a_{n-1}^{\prime}, b\right)$ and $b \in \operatorname{acl}\left(N_{0}, a_{0}, \ldots, a_{n}\right)$ so that $a_{n}^{\prime} \in \operatorname{acl}\left(N_{0}, a_{0}, \ldots, a_{n}\right.$, $\left.a_{0}^{\prime}, \ldots, a_{n-1}^{\prime}\right)$. But $a_{n}^{\prime}$ is distinct from each of $a_{0}, \ldots, a_{n}, a_{0}^{\prime}, \ldots, a_{n-1}^{\prime}$, giving a contradiction to Claim (IV).

So the proof in Case (ii) is also complete, as is the proof of Theorem A.2.

Proof of Proposition 8.6(iii). We return to the context and notation of 8.6. We already know that $X_{i}$ with its ordering $<_{i}$ is strongly $o$-minimal in $\bar{M}^{*}$, and is also a definable subset of $G / G^{00}$. As in Section 8, we use $J$ to denote $G / G^{00}$ as a definable (or interpretable) group in $\bar{M}^{*}$. We will show that $X_{i}$ is untrivial (as a definable strongly $o$-minimal set).

Let $N_{0}$ denote the structure $\bar{M}^{*}$, and let $N$ be a saturated elementary extension. Then $X_{i}(N)$ is $o$-minimal in $N$ and a definable subset of the definable group $J(N)$. In fact we will work over the parameter set $N_{0}$ over which all the data are anyway defined.

Let $\left(a_{i}: i<\omega\right)$ be elements of $X_{i}(N)$ which are algebraically independent over $N_{0}$. For each $i$, let $b_{i}=a_{0} \cdot \ldots \cdot a_{i}$ where the product is in the sense of the group $J(N)$. So $b_{i} \in$ $J(N)$. On the other hand $b_{i} \in \operatorname{dcl}\left(a_{0}, \ldots, a_{i}\right)$ so can be viewed as (is interdefinable with) an element of $X_{i}(N)^{\mathrm{eq}}$. So we can talk about $\operatorname{dim}\left(b_{i}\right)$. Let $n$ be as in 8.5, the dimension of the original $o$-minimal group $G$. By Corollary 8.5 and Proposition 8.6(ii), each element of $J(N)$ is in the definable closure of $n$ elements, each of which is a member of some $o$ minimal definable set (defined over $N_{0}$ ). It follows easily that $\operatorname{dim}\left(b_{i}\right)$ is bounded by $n$. It is easy to see that $\operatorname{dim}\left(b_{i}\right)$ is nondecreasing (as the $a_{i}$ are independent) and $\leq i+1$. Hence for some $m \leq n$, we have $\operatorname{dim}\left(b_{m-1}\right)=\operatorname{dim}\left(b_{m}\right)=m$. Then $b_{m} \in \operatorname{acl}\left(a_{0}, \ldots, a_{m}\right)$, and $b_{m} \notin \operatorname{acl}\left(a_{0}, \ldots, a_{m-1}\right)$ (for otherwise we conclude that $a_{m} \in \operatorname{acl}\left(a_{0}, \ldots, a_{m-1}\right)$ ). Finally $a_{m} \notin \operatorname{acl}\left(b_{m}\right)$. For otherwise, also $b_{m-1}$ is in $\operatorname{acl}\left(b_{m}\right)$, so $b_{m}$ is interalgebraic with $\left(b_{m-1}, a_{m}\right)$, hence has dimension $m+1$, which it does not. We have proved that $X_{i}(N)$ is untrivial. So we can apply Proposition A.2 to obtain 8.6(iii).

Remark A.4. (i) The argument above yields: Suppose that $X$ is a definable strongly $o$ minimal set in a structure $N$ and $X$ definably embeds in a definable group $G$ in $N$ where $G$ has finite thorn rank. Then $X$ is untrivial, so has definable Skolem functions in $N$ after naming parameters.

(ii) A recent paper by Hasson and Onshuus [13] proves that a strongly $o$-minimal definable set $X$ in a structure $N$ is "stably embedded" in $N$. So in Proposition A.2 one may assume that $X$ is the universe of $N$, and the set-up of the proof, although not its content, can be a little simplified. 
Acknowledgments. The second author would like to thank H. Adler, A. Berarducci, C. Ealy and $\mathrm{K}$. Krupiński for helpful conversations and communications around the topics of this paper. $\mathrm{He}$ would also like to thank the Humboldt Foundation for their support of a visit to Berlin in MarchApril 2007 when some of the work on this paper was done.

A first version of this paper was written in October 2007. Both authors would like to thank Itay Kaplan, Margarita Otero, Kobi Peterzil, Marcin Petrykowski, and Roman Wencel, for their detailed reading of parts of the manuscript and for pointing out errors, gaps, and possible improvements. In a new version written in January 2009, several proofs were expanded and clarified, particularly in the current Sections 2, 4, 7 and 8. Substantial changes included removing an old Section 8 on generic compact domination, giving a more complete account of the Vapnik-Chervonenkis theorem and its applications in Section 4, as well as adding an appendix proving the existence of definable Skolem functions in suitable $o$-minimal structures (which is needed for the proof of compact domination in the current Section 8). We would like to thank the referees of this version for their careful reading of and detailed comments on the manuscript, and for encouraging us to further clarify and/or expand on several proofs and explanations in the paper.

The first author thanks for support from the ISF. Research of the second author was supported by a Marie Curie Chair EXC 024052 and EPSRC grant EP/F009712/1.

\section{References}

[1] Adler, H.: Introduction to theories without the independence property. Arch. Math. Logic, to appear

[2] Baisalov, Y., Poizat, B.: Paires de structures $o$-minimales. J. Symbolic Logic 63, 570-578 (1998) Zbl 0910.03025 MR 1627306

[3] Berarducci, A.: o-minimal spectra, infinitesimal subgroups and cohomology. J. Symbolic Logic 72, 1177-1193 (2007) Zbl 1131.03015 MR 2371198

[4] Berarducci, A., Otero, M., Peterzil, Y., Pillay, A.: A descending chain condition for groups definable in o-minimal structures. Ann. Pure Appl. Logic 134, 303-313 (2005) Zbl 1068.03033 MR 2139910

[5] Buechler, S., Pillay, A., Wagner, F. O.: Supersimple theories. J. Amer. Math. Soc. 14, 109-124 (2001) Zbl 0963.03057 MR 1800350

[6] Chernikov, A., Kaplan, I.: Forking and dividing in $N T P_{2}$-theories. J. Symbolic Logic, to appear

[7] Dolich, A.: Forking and independence in $o$-minimal theories. J. Symbolic Logic 69, 215-240 (2004) Zbl 1074.03016 MR 2039358

[8] Edmundo, M., Otero, M.: Definably compact abelian groups. J. Math. Logic 4, 163-180 (2004) Zbl 1070.03025 MR 2114966

[9] Gismatullin, J.: Model-theoretic connected components of groups. Israel J. Math., to appear

[10] Haskell, D.: Hrushovski, E., Macpherson, D.: Definable sets in algebraically closed valued fields: elimination of imaginaries. J. Reine Angew. Math. 597, 175-236 (2006) Zbl 1127.12006 MR 2264318

[11] Haskell, D., Hrushovski, E., Macpherson, D.: Stable Domination and Independence in Algebraically Closed Valued Fields. Lecture Notes in Logic 30, Cambridge Univ. Press (2007) Zbl 1149.03027 MR 2369946

[12] Hart, B., Kim, B., Pillay, A.: Coordinatization and canonical bases in simple theories. J. Symbolic Logic 65, 293-309 (2000) Zbl 0945.03051 MR 1782121

[13] Hasson, A., Onshuus, A.: Embedded o-minimal structures. Bull. London Math. Soc. 42, 64-74. Zbl 1187,2010)03034 MR 2586967

[14] Hrushovski, E.: Valued Fields, Metastable Groups. Draft (2003) 
[15] Hrushovski, E., Kazhdan, D.: Integration in valued fields. In: Algebraic Geometry and Number Theory, Progr. Math. 253, Birkhäuser, Boston, MA, 261-405 (2006) Zbl pre05275304

[16] Hrushovski, E., Peterzil, Y., Pillay, A.: Groups, measures and the NIP. J. Amer. Math. Soc. 21, 563-596 (2008) Zbl 1134.03024 MR 2373360

[17] Hrushovski, E., Peterzil, Y., Pillay, A.: On central extensions and definably compact groups in o-minimal structures. J. Algebra 327, 71-106 (2011) MR 2746030

[18] Ivanov, A.: Strongly determined types and $G$-compactness. Fund. Math. 191, 227-247 (2006) Zbl 1118.03024 MR 2278624

[19] Ivanov, A., Macpherson, D.: Strongly determined types. Ann. Pure Appl. Logic 99, 197-230 (1999) Zbl 0934.03049 MR 1708152

[20] Keisler, H. J.: Measures and forking. Ann. Pure Appl. Logic 45, 119-169 (1987) Zbl 0633.03024 MR 0890599

[21] Keisler, H. J.: Choosing elements in a saturated model. In: Classification Theory (Chicago, IL, 1985), J. Baldwin (ed.), Lecture Notes in Math. 1292, Springer, 165-181 (1987) Zbl 0649.03023 MR 1033028

[22] Lascar, D., Pillay, A.: Hyperimaginaries and automorphism groups. J. Symbolic Logic 66 (2001), 127-143. Zbl 1002.03027 MR 1825177

[23] Newelski, L., Petrykowski, M.: Weak generic types and coverings of groups I. Fund. Math. 191, 201-225 (2006) Zbl 1111.03036 MR 2278623

[24] Onshuus, A., Pillay, A.: Definable groups and compact $p$-adic Lie groups. J. London Math. Soc. 78, 233-247 (2008) Zbl 1153.03015 MR 2427062

[25] Otero, M., Peterzil, Y.: $G$-linear sets and torsion points in definably compact groups. Arch. Math. Logic 48, 387-402 (2009) Zbl 1177.03043 MR 2505431

[26] Peterzil, Y., Pillay, A.: Generics in definably compact groups. Fund. Math. 193, 153-170 (2007) Zbl 1117.03042 MR 2282713

[27] Peterzil, Y., Pillay, A., Starchenko, S.: Definably simple groups in o-minimal structures. Trans. Amer. Math. Soc. 352, 4397-4419 (2000) Zbl 0952.03046

[28] Peterzil, Y., Pillay, A., Starchenko, S.: Simple algebraic and semialgebraic groups over real closed fields. Trans. Amer. Math. Soc. 352, 4421-4450 (2000) Zbl 0952.03047 MR 1779482

[29] Peterzil, Y., Starchenko, S.: A trichotomy theorem for $o$-minimal structures. Proc. London Math. Soc. 77, 481-523 (1998) Zbl 0904.03021 MR 1643405

[30] Peterzil, Y., Steinhorn, C.: Definable compactness and definable subgroups of $o$-minimal groups. J. London Math. Soc. 59, 769-786 (1999) Zbl 0935.03047 MR 1709079

[31] Pillay, A.: On groups and fields definable in o-minimal structures. J. Pure Appl. Algebra 53, 239-255 (1988) Zbl 0662.03025 MR 0961362

[32] Pillay, A.: Geometric Stability Theory. Oxford Univ. Press (1996) Zbl 0871.03023 MR 1429864

[33] Pillay, A.: Type-definability, compact Lie groups, and $o$-minimality. J. Math. Logic 4, 147 162 (2004) Zbl 1069.03029 MR 2114965

[34] Poizat, B.: A Course in Model Theory. Springer (2000) Zbl 0951.03002 MR 1757487

[35] Shelah, S.: Classification theory for elementary classes with the dependence property-a modest beginning. Sci. Japon. 59, 265-316 (2004) Zbl 1081.03031 MR 2062198

[36] Shelah, S.: Dependent first order theories, continued. Israel J. Math. 173, 1-60 (2009) Zbl 1195.03040 MR 2570659

[37] Shelah, S.: Minimal bounded index subgroup for dependent theories. Proc. Amer. Math. Soc. 136, 1087-1091 (2008) Zbl 1144.03026 MR 2361885

[38] Shelah, S.: Definable groups and 2-dependent theories. Preprint (2007) 
[39] Vapnik, V. N., Chervonenkis, A. Ya.: On the uniform convergence of relative frequencies of events to their probabilities. Theory Probab. Appl. 16, 264-280 (1971) Zbl 0247.60005 MR 0288823

[40] Wagner, F. O.: Simple Theories. Kluwer (2000) Zbl 0948.03032 MR 1747713 